\title{
Dynamics of Oscillator Chains
}

\author{
Allan J. Lichtenberg ${ }^{1}$, Roberto Livi ${ }^{2}$, Marco Pettini ${ }^{3}$ and Stefano Ruffo ${ }^{4}$ \\ 1 Electrical Engineering and Computer Science Department University of \\ California, Berkeley, CA 94720-1770, USA \\ ajl@eecs.berkeley.edu \\ 2 Dipartimento di Fisica and CSDC, Università di Firenze via G. Sansone 1, 50019 \\ Firenze, Italy \\ livi@fi.infn.it \\ 3 INAF - Osservatorio Astrofisico di Arcetri Largo Enrico Fermi 5, 50125 Firenze, \\ Italy \\ pettini@arcetri.astro.it \\ 4 Dipartimento di Energetica "S. Stecco" and CSDC, Università di Firenze, and \\ INFN, via s. Marta 3, 50139 Firenze, Italy \\ stefano.ruffo@unifi.it
}

\begin{abstract}
The Fermi-Pasta-Ulam (FPU) nonlinear oscillator chain has proved to be a seminal system for investigating problems in nonlinear dynamics. First proposed as a nonlinear system to elucidate the foundations of statistical mechanics, the initial lack of confirmation of the researchers expectations eventually led to a number of profound insights into the behavior of high-dimensional nonlinear systems. The initial numerical studies, proposed to demonstrate that energy placed in a single mode of the linearized chain would approach equipartition through nonlinear interactions, surprisingly showed recurrences. Although subsequent work showed that the origin of the recurrences is nonlinear resonance, the question of lack of equipartition remained. The attempt to understand the regularity bore fruit in a profound development in nonlinear dynamics: the birth of soliton theory. A parallel development, related to numerical observations that, at higher energies, equipartition among modes could be approached, was the understanding that the transition with increasing energy is due to resonance overlap. Further numerical investigations showed that time-scales were also important, with a transition between faster and slower evolution. This was explained in terms of mode overlap at higher energy and resonance overlap at lower energy. Numerical limitations to observing a very slow approach to equipartition and the problem of connecting high-dimensional Hamiltonian systems to lower dimensional studies of Arnold diffusion, which indicate transitions from exponentially slow diffusion along resonances to power-law diffusion across resonances, have been considered. Most of the work, both numerical and theoretical, started from low frequency (long wavelength) initial conditions.

Coincident with developments to understand equipartition was another program to connect a statistical phenomenon to nonlinear dynamics, that of understanding classical heat conduction. The numerical studies were quite different, involving the excitation of a boundary oscillator with chaotic motion, rather than the excitation of
\end{abstract}


the entire chain with regular motion. Although energy transitions are still important, the inability to reproduce exactly the law of classical heat conduction led to concern for the generiticity of the FPU chain and exploration of other force laws. Important concepts of unequal masses, and "anti-integrability," i.e. isolation of some oscillators, were considered, as well as separated optical and acoustic modes that could only communicate through very weak interactions. The importance of chains that do not allow nonlinear wave propagation in producing the Fourier heat conduction law is now recognized.

A more recent development has been the exploration of energy placed on the FPU or related oscillator chains in high-frequency (short wavelength) modes and the existence of isolated structures (breathers). Breathers are found as solutions to partial differential equations, analogous to solitons at lower frequency. On oscillator chains, such as the FPU, energy initially in a single high-frequency mode is found, at higher energies, to self-organize in oscillator space to form compact structures. These structures are "chaotic breathers," i.e. not completely stable, and disintegrate on longer time-scales. With the significant progress in understanding this evolution, we now have a rather complete picture of the nonlinear dynamics of the FPU and related oscillator chains, and their relation to a wide range of concepts in nonlinear dynamics.

This chapter's purpose is to explicate these many concepts. After a historical perspective the basic chaos theory background is reviewed. Types of oscillators, numerical methods, and some analytical results are considered. Numerical results of studies of equipartition, both from low-frequency and high-frequency modes, are presented, together with numerical studies of heat conduction. These numerical studies are related to analytical calculations and estimates of energy transitions and timescales to equipartition.

\subsection{Historical Perspective and Background Theory}

\subsubsection{Motivation and Counter Intuitive Numerical Results}

In the early 1950s, considering what numerical investigations could be performed on a first generation digital computer at Los Alamos National Laboratory, Enrico Fermi suggested to Stanislaw Ulam and John Pasta that the foundations of statistical mechanics could be explored. He proposed using a chain of coupled slightly nonlinear oscillators to show that the nonlinearity would lead to equipartition of energy among the degrees of freedom. The model used in the studies was a discretization of a nonlinear spring which to quartic order is given by the normalized Hamiltonian

$$
H=\sum_{i}^{N}\left[\frac{p_{i}^{2}}{2}+\frac{\left(q_{i+1}-q_{i}\right)^{2}}{2}+\alpha \frac{\left(q_{i+1}-q_{i}\right)^{3}}{3}+\beta \frac{\left(q_{i+1}-q_{i}\right)^{4}}{4}\right]
$$

with $N$ unit masses and unit harmonic coupling. The oscillator chain is known as the Fermi-Pasta-Ulam (FPU) model. The original simulations were done with only the $\alpha$ term present (FPU- $\alpha$ model) or only the $\beta$ term present 
(FPU- $\beta$ model); most subsequent simulations and analysis were done with the FPU- $\beta$ model. With periodic endpoints the chain is translationally invariant, but the original simulations, as with much subsequent work, considered fixed endpoints, related to a physical finite string. Without nonlinear terms the coordinates can be transformed to uncoupled normal modes, such that the energy is always confined to the initial modes in which it is placed. For small nonlinearities (energy in the nonlinear terms small compared to the energy in the linear terms) it is logical to place the energy in a mode (or modes) of the linear system (quasi-modes of the nonlinear system) and observe the subsequent behavior of the mode energies subject to the laws of motion described by the Hamiltonian in (2.1). The initial numerics, programmed by Mary Tsingou for the FPU- $\alpha$ chain with fixed ends and $N-1$ moving particles, and with all the energy placed in the first harmonic $(k=1)$ of the harmonic normal modes

$$
Q_{k}=\sqrt{\frac{2}{N}} \sum_{i=1}^{N} q_{i} \sin \left(\frac{\pi i k}{N}\right)
$$

(with $N=32$ and $\alpha=1 / 4$ ) gave, for example, the result shown in Fig. 2.1 for $Q_{1}=4\left(E=E_{1}=0.077\right)$. The initial energy was transferred primarily into the first four modes, with an approximate recurrence (within a few percent) occurring in a time $\omega_{1} t / 2 \pi=157$ fundamental periods. Similar results were obtained for other initial conditions for both the $\alpha$ and $\beta$ models,

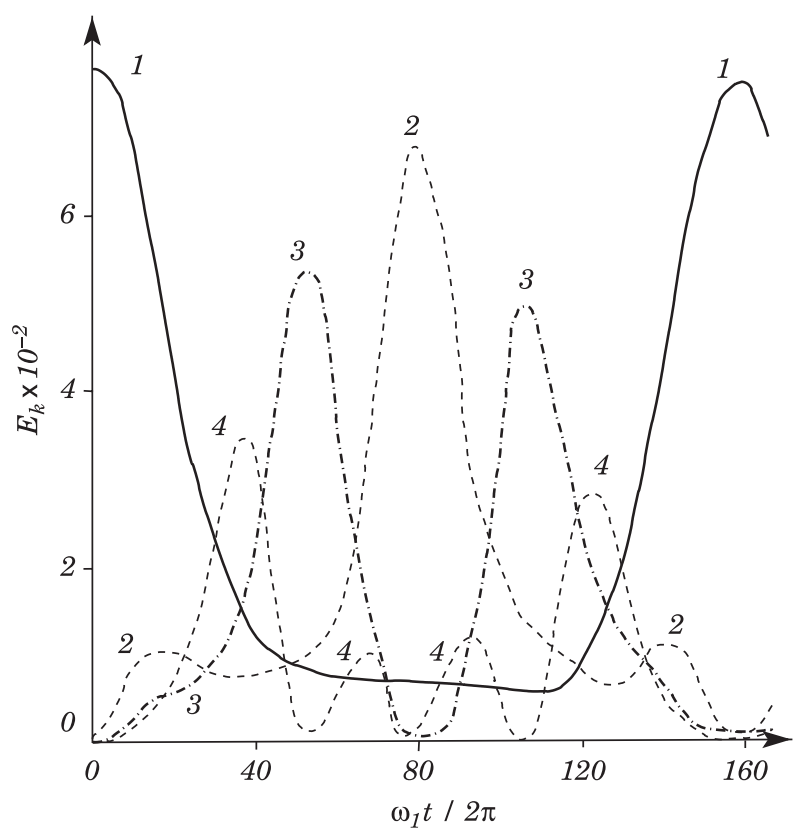

Fig. 2.1. FPU original mode oscillations 
with the results presented in a Los Alamos report in 1955. Unfortunately, the untimely death of Fermi, prevented regular publication until the work appeared in Fermi's collected works [1]. The simulations did not answer the question of whether equipartition would ultimately be obtained, as was predicted from general dynamical principles of the nonexistence of global isolating integrals, see Poincaré [2], and the inferences used to support the concept of ergodicity by Fermi himself $[3,4]$. Most of the near-term response to the unexpected result was to try to explain the recurrences. Using perturbative analysis Ford [5] and Jackson [6] obtained oscillations of the first few harmonics, with Jackson's approach, using nonlinearly perturbed frequencies, giving results, including the recurrence times, quite similar to the numerical observations. However, the perturbation procedures are nonconvergent, so no conclusions can be drawn from them about long-time behavior. There has been a significant body of literature concerned with these recurrences and methods of analysis. Generalizations, for example, have considered energy initially in an arbitrary mode (rather than the lowest frequency mode), the major couplings identified, and the effect of various numbers of modes used in analyzing the dynamics [7]. It was found, for example, that there is an induction period, i.e. a time during which there is little change in mode energy, if the energy is initially placed in a high-frequency mode, a condition later observed and qualitatively explained (see below).

\subsubsection{Chaos Theory: KAM Isolation, Arnold Diffusion, Lyapunov Exponents, KS Entropy}

Parallel to the developments, described above, for a high-dimensional Hamiltonian system, there were developments in low-dimensional Hamiltonian dynamics that informed the oscillator-chain results, and ultimately were informed by those results. In particular, the KAM theorem for coupled degrees of freedom $[8,9,10]$ indicated that the generic case was a divided phase space with regular and chaotic orbits interspersed. Numerical observations, in a surface of section of a particular two degree of freedom system (the Hénon and Heiles potential), indicated mostly regular orbits at low energy, with the chaotic portion of the phase space increasing rather abruptly over a small range of increasing energy, until most of the phase space is chaotic [11]. A practical explanation of this rather abrupt increase was that local resonances between frequencies of the two freedoms, which modified the structure of the phase space in their neighborhood, would overlap with increasing energy, producing large areas of chaotic motion $[12,13]$. For systems with three or more degrees of freedom KAM surfaces cannot isolate chaotic regions: leading to the possibility of "diffusion", in the sense that there are initial data which can reach points in phase space that are arbitrarily far, although such data have a small microcanonical measure when the nonintegrable perturbation is small [14]. Furthermore, a heuristic understanding of a many-dimensional system with weak coupling, backed up by simulations (see [15], Sect. 6.5), indicated 
the fraction of the phase volume that is stochastic continually increases with increasing number of freedoms $N$ [16]. Another relevant theoretical result is that the upper bound on the rate of Arnold diffusion is proportional to

$$
\exp \left(\frac{1}{\mu^{q}}\right)
$$

where $\mu$ is a perturbation parameter [17] and an "optimal convergent" perturbation calculation gave a value of $q \approx 1 / N[18,19]$. This result would indicate that the diffusion becomes large if $\mu^{1 / N} \approx 1$, but the result, being of perturbative type, does not extend to so large a perturbation. Other heuristic forms will be used to estimate the diffusion rate in later Sections. Another development in low-dimensional chaos, that would inform the high-dimensional oscillator chain research, was the study of the three-particle Toda lattice [20], with Hamiltonian

$$
H=\frac{p_{1}^{2}+p_{2}^{2}+p_{3}^{2}}{2}+\exp \left(-\left(q_{1}-q_{3}\right)\right)+\exp \left(-\left(q_{2}-q_{1}\right)\right)+\exp \left(-\left(q_{3}-q_{2}\right)\right)-3
$$

which corresponds to three particles moving on a ring with exponentially decreasing repulsive forces between them. In addition to the energy, there is a relatively obvious isolating integral, namely the total momentum, reducing the motion to two degrees of freedom. The Henon-Heiles potential [11] is a truncation of the Toda lattice. However, a surface of section of (2.4), calculated numerically, showed no chaos [21] and Hamiltonian (2.4) was subsequently proved to have a third invariant and thus was integrable, i.e. had nonchaotic phase-space trajectories [22].

In order to obtain equipartition it is sufficient for the dynamics to be "ergodic" on the energy shell, i.e. microcanonical averages over a given energy surface and time averages over motions taking place over the same energy surface must be equal. However, since it is physically relevant that the convergence to equipartition should occur on a finite time, and be possibly fast, the stronger dynamical property of "mixing" could be required. A direct numerical check of both "ergodicity" and "mixing" is impossible in systems with many degrees of freedom. However, if all trajectories are chaotic and, hence, on the average exponentially separating, positive Kolmogorov-Sinai (KS) entropy $[23,24]$ and exponentially fast "mixing" follow as a consequence. Therefore, an obvious quantity to be examined is the largest Lyapunov exponent, giving the average separation rate between nearby trajectories as

$$
\lambda=\lim _{t \rightarrow \infty} \frac{1}{t} \ln \frac{\|\xi(t)\|}{\|\xi(0)\|}
$$

where $\xi(t)$ is the tangent vector whose time evolution is described by the tangent dynamics equation described in Sect. 2.3. A positive value of $\lambda$ indicates exponential separation of initially close trajectories, i.e. chaos. A difficulty of realistic Hamiltonian systems is that, in generic conditions, the energy shell 
is "divided" into chaotic and ordered trajectories, and hence "mixing" cannot occur everywhere in phase-space. Then, the qualitative statement might be that, if almost all of the energy surface is characterized by an invariant distribution that has a positive KS entropy, then, for all practical purposes, equipartition will be reached. The numerical calculation of Lyapunov exponents has been used extensively to test for chaotic motion, particularly after the numerical techniques were formalized [25]. It was logical that the method would be applied to the FPU chain, and became an important element in the numerical investigations in the 1980s and beyond, as discussed in more detail below.

For a more complete introduction to these topics see [15]: Sect. 3.2 (KAM Theory); Sects. 5.2 and 5.3 (concepts of stochasticity) and Sects. 6.1 and 6.2 (Arnold diffusion). See also [13] and, more rigorously, [26].

\subsubsection{Geometrization of Hamiltonian Dynamics}

Without attempting to be exhaustive, a few historical comments might be helpful to place the recent contributions about the geometrical approach to dynamics which are reviewed in the present Chapter, in a more general context.

The idea of looking at the collection of solutions of the Newton's equations of motion from a geometric point of view dates back to Poincaré and to the development of the qualitative theory of differential equations. Tackling the famous problem of the integrability of the three-body problem, Poincaré discovered that generic classical Hamiltonian systems, in spite of their deterministic nature, lack predictability because of their extreme sensitivity to the initial conditions. Such an instability of classical dynamics originates in homoclinic intersections, which Poincaré described in his Méthodes Nouvelles de la Mécanique Céleste [2] without "even attempting to draw" them. The method was later developed by Cartan among others, using what is now called symplectic geometry [27]. Although of undeniable elegance, symplectic geometry is not very helpful to advance our knowledge about the regions in phase space where the dynamics is unstable. The name of Poincaré, together with that of Fermi, is also associated with an important theorem about the nonexistence of analytic integrals of motion, besides energy, for generic nonlinear Hamiltonian systems describing at least three interacting bodies [3, 4]; this is the origin of the concept of topological accessibility of the whole constant energy hypersurface of phase space with high degree of freedom systems, with generic initial conditions.

In the 1940s, a qualitatively new attempt was made to make use of geometric concepts to relate Newtonian dynamics with statistical mechanics. Krylov [28] showed in for the first time the existence of a relationship between dynamical instability (seen as the exponential amplification of small deviations in the initial conditions of a collection of colliding objects representing idealized atoms in a gas) and phase space mixing. Phase mixing is a stronger 
property than ergodicity and is far more relevant to physics than ergodicity. In fact, while ergodicity assures the equality of time and phase space averages of physical quantities, phase mixing addresses the rate of approach to ensemble averages in a finite time. In modern terms, Krylov realized the necessity of chaotic dynamics to obtain fast phase mixing for the physically relevant observables and to make the connection between dynamics and statistical mechanics stronger. But Krylov also has the great historical merit of having attempted to bridge the dynamical foundations of statistical mechanics with a powerful field of mathematics, Riemannian differential geometry. Krylov knew mathematical results, concerning the properties of geodesic flows on compact negatively curved manifolds, by Hadamard [29], Hedlund [30] and Hopf [31]. He envisioned their potential interest to physics, once Newtonian dynamics is rephrased in terms of Riemannian geometric language. Such a possibility was well known since the beginning of the century, mainly due to the work of Levi-Civita; in particular that the principle of stationary action entails the close connection of a classical mechanical flow with a geodesic flow in a configuration space endowed with a suitable metric. Krylov's efforts concentrated on the analysis of the properties of physical systems which move in negatively curved regions in configuration space. For example, he discussed how the presence of an inflection point in the Lennard-Jones potential could influence the dynamics of a dilute gas (through the appearance of regions of negative scalar curvature in configuration space) and its ensuing strong instability. These attempts have been very influential on the development of the so-called abstract ergodic theory, where Anosov flows [32] (e.g., geodesic flows on compact manifolds with negative curvature) play a prominent role. Ergodicity and mixing of these flows have been thoroughly investigated. To give an example, Sinai proved ergodicity and mixing for two hard spheres by just showing that such a system is similar enough to a geodesic flow on a negatively curved compact manifold [33]. Krylov's intuitions have been worked out further by several physicists amongst whom we cite those of $[34,35,36,37,38,39,40]$. They discovered, much to their surprise, that geodesic flows associated with physical Hamiltonians do not live on negatively curved manifolds, despite their chaoticity. Only a few exceptions are known, in particular two low-dimensional models $[35,36,41]$, where chaos is actually associated with hyperbolicity due to everywhere negatively curved manifolds. In fact, for certain models the regions of negative curvature of the mechanical manifolds apparently shrink by increasing the number $N$ of degrees of freedom, thus reducing the frequency of the visits of negatively curved regions.

This somewhat biased search for negative curvature has been the main obstacle to an effective use of the geometric framework originated by Krylov to explain the source of chaos in Hamiltonian systems. On the other hand, it is true that the Jacobi equation, which describes the stability of a geodesic flow, is in practice only tractable on negatively curved manifolds. Formidable mathematical difficulties are encountered in treating the (in)stability of geodesic flows on manifolds of nonconstant and not everywhere negative curvature. 
Moreover, for this kind of problem, intuition can hardly help. However, the advent of computers has been of invaluable help. As a matter of fact, during the last few years an interplay between analytic methods and numerical simulation has made it possible to overcome the difficulties, showing that the Riemannian geometric approach can be applied to dynamical systems of interest to statistical mechanics, field theory, and condensed matter physics [42]. This has extended the domain of application of geometric techniques, and has also introduced a new point of view about the origin of chaos in Hamiltonian systems, as well as new methods to describe and understand it, "new" in a sense that will be made clear in Sect. 2.4.7.

A more detailed exposition of the geometric method and its application to calculating Lyapunov exponents, which we will be summarizing in Sects. 2.4.7 and 2.8.2, can be found in [42]. See also mathematical expositions in [27, 28].

\subsubsection{Development of Soliton Theory}

It is somewhat ironical that the most celebrated result that came out of the investigation of the FPU chain did little to resolve Fermi's original question of whether or not the nonlinearity would lead to equipartition among the degrees of freedom. In an attempt to understand the apparent stability of the recurrences Norman Zabusky and Martin Kruskal [43, 44] found a Taylor expansion of the discreteness, valid for long wavelength modes, that recovered partial differential equations, different from the original nonlinear spring which produced the discretized chain of oscillators. The resulting equations are the Korteveg-de Vries (KdV) equation for the FPU- $\alpha$ chain and the modified Korteveg-de Vries (mKdV) equation for the FPU- $\beta$ chain $[43,44]$. The latter chain, with appropriate normalizations, gave the standard form

$$
u_{\tau}+12 u^{2} u_{\xi}+u_{\xi \xi \xi}=0
$$

where $\tau=h^{3} t / 24, \xi=x-h t, h=L / N, L$ with the length of the string and $N$ the number of oscillators. Nonlinear equations of this and related types had been known to have stable traveling solutions, where the dispersion and nonlinearity balance to produce constant amplitude and propagation velocity. An arbitrary initial condition, such as the lowest linear mode on the FPU- $\beta$ chain, breaks up initially into a set of structures each having a steady traveling solution with its own velocity. Remarkably, these structures are sufficiently stable that they pass through one another without breaking up, and the observed recurrences can be interpreted in terms of their superpositions. But these results do not improve on the best perturbation calculations, and are clearly limited to long-wavelength (low-frequency) modes by the approximations which led to (2.6). Partial differential equations, like (2.6), have an infinite number of freedoms, such that general integrability from arbitrary initial conditions requires an infinite number of invariants of the motion. The real excitement came when it was shown that such an infinite set exists for (2.6), 
and the new field of soliton theory and applications was born, which would take us far from the subject at hand. A final note, which is important to our overall understanding, is that a single initial nonlinear mode solution of the $\mathrm{mKdV}$ equation was found to become unstable as the energy is increased. A linearization around the nonlinear structure predicted the unstable wave numbers and growth rates, and showed that the values correspond to the observed mode growth for the same discretized structure on the FPU- $\beta$ oscillator chain $[45,46]$. The result in which one soliton decomposes into a finite number is not inconsistent with general soliton theory. The instability will give us insight into some later results.

\subsubsection{Resonance Overlap Explanations}

Using the concept of mode overlap to estimate the transition between regular and chaotic motion Felix Izrailev and Boris Chirikov obtained estimates for mode overlap both for low- and high-frequency modes [47]. Although there are various approximations required to obtain results, a simple numerical estimate can be made by equating the nonlinear frequency shift $\Delta \omega_{k}$ to the mode spacing $\delta \omega_{k}$, i.e. setting $\Delta \omega_{k} / \delta \omega_{k} \approx 1$. The mode overlap estimate from this approximation, in terms of energy density, is

$$
\varepsilon_{k}=\frac{E_{k}}{N}= \begin{cases}4 /(3 \beta k), & k \ll N \\ 2 k^{2}(N-k) /\left(3 \beta N^{2}\right), & (N-k) \ll N .\end{cases}
$$

The result for long-wavelength modes, $k \ll N$, is not a necessary condition, as seen in many subsequent numerical experiments, but approximates another transition, discussed below, between weak and strong stochasticity (the SST). The result for short-wavelength modes, $(N-k) \ll N$, is neither necessary nor sufficient. It predicts easy overlap at short wavelengths due to mode crowding, while numerical simulations show consistently that, from a practical point of view, equipartition is more readily obtained from long-wavelength than from short-wavelength initial conditions. General theoretical arguments as to the accessibility of modes has been advanced to show that this is the case $[48,49]$.

We discuss a resonance overlap criterion, as presently used, in Sect. 2.4.1. The concept, initially proposed by Chirikov for two degrees of freedom and reviewed by him, including higher dimensionality, [13], can also be found in [15], Chap. 4.

\subsubsection{Numerical Methods}

The straightforward method of computing Lyapunov exponents, using (2.5), particularly the largest exponent, was a powerful numerical tool for statistically investigating the dynamical properties of oscillator chains. Another very useful statistical quantity is the information entropy 


$$
S=-\sum_{k} e_{k} \ln e_{k}
$$

with $e_{k}=E_{k} / \sum_{k} E_{k}$, such that $S=0$ if all the energy resides in a single mode, and has a maximum $S=\ln N$ if the energy is uniformly distributed among all modes. By using (2.5) and (2.8), detailed numerical investigations were carried out among investigators in Florence [50, 51, 52, 53], starting from long-wavelength modes of the FPU- $\beta$ system, obtaining the variation of $\lambda$ and $S$ with energy density $\varepsilon=E / N$. They found a distinct break in the behavior between weak stochasticity at lower values of $\varepsilon$, having strong power-law dependencies of $\lambda$ and $S$ on $\varepsilon$, and strong stochasticity at higher $\varepsilon$ with weak $\varepsilon$ dependence. The transition (SST) is qualitatively related to the mode overlap criterion (2.7). Note that $\varepsilon$ is not necessarily a small quantity.

\subsubsection{Methods of Analysis and Numerical Results}

It is clear from the phase space description of high-dimensional systems that mode overlap is not necessary to obtain positive Lyapunov exponents. Most generic initial conditions will lie in stochastic layers, exhibiting $\lambda>0$. The question becomes what determines the rate of energy diffusion between the degrees of freedom? One approach to this problem is to isolate a few of the most closely coupled modes and determine if their resonant interaction results in chaos that can then couple to other resonances. This was done, as described previously, for low-dimensional chaos to understand the exponentially slow Arnold diffusion. For high-dimensional systems the situation is more complicated with rapid diffusion across overlapped resonances and slow Arnold diffusion along resonance (see [15], Chap. 6, for a detailed discussion). The method of isolating a few interacting resonances and then calculating their coupling to the larger phase space was used for another oscillator chain, a discretized sine-Gordon equation, to explore the transition from power-law (numerically observable) equipartition rates with varying $\varepsilon$, to exponentially slow (not numerically observable) rates [54]. It was also found, using this approach and comparison with numerics, that short-wavelength mode interactions required considerably higher energy to produce chaos. The method was then applied to the FPU- $\beta$ chain, in more detail, specifically investigating the process by which stochastic interaction between a few long-wavelength modes was transferred to short-wavelength modes and calculating a transition between exponentially slow and power-law scaling of the energy transfer [55]. At about the same time, there was considerable attention given to determining the scaling of the equipartition time $T_{\text {eq }}$ with $\varepsilon$, in the power-law regime, finding $T_{\text {eq }} \propto \varepsilon^{-3}[56,57,58]$ with the latter references giving a heuristic calculation of this scaling. The numerics and method of estimation will be given in Sects. 2.5 and 2.8, respectively. Other authors have fitted the data to a "stretched exponential", $T_{\text {eq }} \propto \exp \left(-\varepsilon^{1 / 4}\right)$ [59], obtaining a better agreement over a wider $\varepsilon$ range, but without any theoretical underpinning. Indeed, the 
reason for this scaling has not been explicitly explained, nor has its relation to the power-law scaling. It is very likely that, as $\varepsilon$ is decreased, longer and longer time-scales come into play and, therefore, no definite functional form will be able to fit the increase of the time-scale over the full small $\varepsilon$ range. The scaling $\lambda \propto \varepsilon^{2}$, detected at $\varepsilon$ smaller than the threshold value of the SST transition $(\varepsilon \approx 1)$, has also not been specifically related to the $T_{\text {eq }} \propto \varepsilon^{-3}$ scaling in the same $\varepsilon$ range. The scaling at higher $\varepsilon$, that is at $\varepsilon$ larger than the threshold value of the SST transition, has been heuristically determined using a random matrix approximation for the tangent dynamics, intuitively suggesting that above the SST chaos is fully developed [53]. The scalings of $\lambda$ with $\varepsilon$ can now be determined, analytically, by considering the geometry of the phase space near equipartition. Making suitable assumptions about the geometry of mechanical manifolds, the scaling of $\lambda$ with $\varepsilon$, both below and above the SST transition and the value of $\varepsilon$ at the transition has been theoretically calculated in agreement with numerical findings [42, 60, 61]. Although the method was developed to understand the FPU- $\beta$ scaling, it is applicable to oscillator chains with various force laws, as can be found in the referenced works. The mathematical procedure is outlined in Sects. 2.4.7 and 2.8.2.

\subsubsection{Comparison of Different Oscillator Chains}

Although the FPU- $\beta$ oscillator chain has received most of the attention, there has been, from the beginning, interest in other force laws. The cubic potential in the FPU- $\alpha$ model is more conducive to using expansion procedures to obtain analytic estimates [62], and also, the form with periodic boundary conditions and appropriately chosen $\alpha$ is a third order truncation of the Toda lattice potential, which is integrable. However, the FPU- $\alpha$ is not energy renormalizable with varying $\alpha$ (does not scale with $\alpha E$ ), and furthermore suffers from the problem of unbounded trajectories at high energy. Nevertheless, comparison with the FPU- $\beta$ dynamics has added considerably to our overall understanding. If the finite time version of the Lyapunov exponent (2.5) is calculated for the $N$-particle Toda lattice and its FPU- $\alpha$ approximation, the two exponents decrease, without separation, until some "induction time" or "trapping time" $\tau_{\mathrm{T}}(\varepsilon)$, is reached, after which $\lambda_{\mathrm{FPU}-\alpha}$ attains a constant value, while $\lambda_{\text {Toda }}$ continues to decay, as it must for an integrable system [61]. Plotting $\tau_{\mathrm{T}}(\varepsilon)$, with $N$ as a parameter, in the weak stochasticity regime, it was found in [61] that $\tau_{\mathrm{T}} \propto \varepsilon^{-2}$, which is different from the $T_{\text {eq }} \propto \varepsilon^{-3}$ scaling found both for the FPU $-\alpha$ and the FPU- $\beta$ systems, i.e. the trapping time and the equipartition time scale differently with $\varepsilon$. A transition at some small $\varepsilon$ to a rapid increase in $\tau_{\mathrm{T}}$ with decreasing $\varepsilon$, with the transition value a function of $N$, was also observed and interpreted as a transition to regular motion. This phenomenon had been observed earlier in the discretized sine-Gordon system and interpreted in a similar fashion [54]. However, subsequent work with the FPU- $\beta$ system elicited a different interpretation, that the transition was to the exponentially slow form of Arnold diffusion [55]. These different interpretations 
have not been theoretically reconciled. The Hamiltonian containing both cubic and quartic nonlinearities, as in (2.1), has also been investigated [63].

The contrast of the FPU oscillator chain with other types of chains has also led to considerable insight and some additional puzzles. The class of KleinGordon chains, with on-site potentials, are similar to the FPU, but more complicated, both because they lack the FPU translational invariance and because they have an additional parameter whose scaling must be determined. In addition to the sine-Gordon version, a closer comparison with the FPU- $\beta$ chain employs a Klein-Gordon on-site potential having quadratic and quartic terms, with Hamiltonian

$$
H=\sum_{i=1}^{N} \frac{p_{i}^{2}}{2}+\frac{\left(q_{i+1}-q_{i}\right)^{2}}{2}+\frac{m q_{i}^{2}}{2}+\frac{\beta q_{i}^{4}}{4},
$$

which is often called the $\phi^{4}$ model to distinguish the quartic nonlinearity from other Klein-Gordon potentials. In the first comprehensive numerical comparison of the two systems, (2.1) and (2.9), some physical differences were observed and, qualitatively, understood. In particular, at a given $\varepsilon$, the $\phi^{4}$ took significantly shorter time to obtain equipartition from long-wavelength mode initial conditions and significantly longer time from short-wavelength modes, than the FPU [64]. The fact that from short wavelengths it was generally a longer process to obtain equipartition was remarked in that early work, but little background theory had been done for these initial conditions. A later comparison for the long wavelengths provided a more complete numerical study and was able to explain quantitatively these differences [65]. We will present the numerical comparisons in Sect. 2.5 and outline the supporting theory in Sect. 2.8. The understanding of the results from short wavelengths awaited the development of new theoretical concepts, as given below. Before considering this subject we note that the emphasis on energy density, holding $\varepsilon$ constant as $N$ is varied, i.e. $E \propto N$, is not always the relevant way to look at a problem, as seen in calculating resonance overlap [55]. The case in which $E$ is held fixed as $N$ is varied has been used to analytically calculate stochasticity thresholds of the FPU and $\phi^{4}$ models [66].

\subsubsection{Dynamics at Short Wavelengths: Chaotic Breathers}

Following the original numerical work, most numerical studies examined the evolution from long-wavelength (low-frequency) modes in which neighboring oscillators are nearly in phase. Zabusky and Deem [67] were the first to consider the case in which the energy is put into a high-frequency mode. In their early work, the zone-boundary mode was excited with an added spatial modulation for the FPU- $\alpha$ model. Our main concern here will be the FPU- $\beta$ model, and spatial modulation of the mode is spontaneously created by modulational instability. Budinsky and Bountis [68] found that the zone-boundary $\pi$-mode, 
i.e. the mode with $180^{\circ}$ phase shift between neighboring oscillators of the onedimensional FPU lattice is unstable above a given energy threshold $E_{\mathrm{c}}$ which scales like $1 / N$. This result was later confirmed by Flach [69] and Poggi and Ruffo [70], who also obtained the exact numerical factor relating $E_{\mathrm{c}}$ to $1 / N$. These results were obtained using a direct linear stability analysis around the periodic orbit corresponding to the $\pi$-mode. Similar methods have been more recently applied to other modes and other FPU potentials by Chechin $[71,72]$ and Rink [73]. A technique which allows for a more general exploration of the dynamics starting from short wavelengths is to follow an envelope function of the oscillators defined by $\psi_{i}=(-1)^{i} q_{i}$. Since the main phase variation of the oscillator amplitudes $q_{i}$ vary by nearly $\pi$ from one oscillator to the next, the $\psi_{i}$ vary slowly; a Taylor expansion of the envelope function in the oscillator space can produce a differential equation whose equilibrium properties, stability, and nonlinear effects can be explored (see Sect. 2.4.5). A formula for $E_{\mathrm{c}}$, valid for all $N$, has been obtained in Refs. $[74,75,76,77]$ in the rotating wave approximation (RWA) given in (2.86). Besides calculating the energy threshold, the growth rates of mode amplitudes were obtained. The application to the Klein-Gordon lattices was first studied by Kivshar and Peyrard [78], following an analogy with the Benjamin-Feir instability in fluid mechanics [79]. A different approach to describe this instability had been previously introduced by Zakharov and Shabat [80], who studied the associated nonlinear Schrödinger equation in the continuum limit. Using that method for the FPU equations of motion, the instability boundary was found by Berman and Kolovskii [81] in the so-called "narrow-packet" approximation. Detailed numerics over longer times were obtained for the FPU model by Pettini [64] and for the discretized sine-Gordon equation by Goedde [54], both indicating that, for a given energy, short-wavelength (high-frequency) modes required longer times to reach equipartition than long-wavelength modes. At about the same time it was demonstrated that stable intrinsic localized modes (ILMs) could exist for anharmonic periodic structures [82]. However, from more general high-frequency initial conditions there was a tendency to form ILMs but they were not stable, breaking up and ultimately decaying toward equipartition [75, 83].

The existence of ILMs (also called breathers) on periodic chains and the complex behavior of more arbitrary high-frequency initial conditions has led to extensive study of these structures to understand their stability. A comprehensive review of these studies would lead us far from the main topic of this review (see [84] for a review and further references). The breathers can be stationary or moving, and, like low-frequency solitons, can pass through one another. Whether energy is exchanged in such an interaction depends on the system's stability properties. ILMs that are not exact solutions of the underlying system generally exchange energy, and in a particular process have been shown to transfer energy from the smaller to the larger breather [85]. This phenomenon is also observed numerically for a Klein-Gordon chain [86] and for the FPU- $\beta$ chain $[87,88]$. For fixed end-points, as in the original FPU studies and much subsequent work, a clearly defined instability boundary 
cannot be calculated for a discrete chain. Nevertheless, as described below, some approximate results are available.

The four-mode resonance overlap criterion for a stochasticity transition, described in Sect. 2.4.1 for low-frequency modes, has also been used for highfrequency modes for the discretized sine-Gordon chain [54]. It predicted the increased stability for these modes, as found numerically. Another approach to a reduced problem is to represent the main-energy containing oscillator and the immediate neighboring oscillators as a three degree of freedom system from which a mapping can be obtained [89]. In this reduced phase space, depending on the energy and the action, one observes both regular and chaotic regions. The chaotic regions are sufficient to indicate chaos in the larger system, but do not give a time-scale for equipartition to be approached. The regular regions may also be chaotic in the larger system, but are generally more weakly so. Note that the mapping presentation uses initial conditions close to those of a breather, which is narrow in oscillator space, and therefore has a broad distribution of energies in mode space. Contrarily, the mode presentation starts with a narrow distribution in mode space and therefore a broad distribution in oscillator space. The technique employing the envelope equations and the RWA has been used to describe longer time effects, as well as instability boundaries both for periodic boundary conditions at low energies [90, 91] and fixed boundary conditions at both low and high energies $[92,93,94]$, and used to compare the dynamics of the FPU and $\phi^{4}$ chains [94].

The general picture that has emerged is that if the energy is placed in a high-frequency mode or modes for which neighboring oscillators are primarily out of phase, a complicated dynamics ensues, which consists of three stages. First, there is an initial stage in which, for sufficiently high energy, the mode breaks up into a number of breather-like structures. Second, on a slower time-scale, these structures coalesce into one large unstable structure, called a "chaotic breather" (CB). Since a single large CB closely approximates a stable breather, a third and final decay stage, toward equipartition, can be very slow. One does not know whether there exists any true energy threshold to achieve equipartition, although there appears to be some numerical evidence for such a threshold in the discretized sine-Gordon system. However, as discussed extensively with respect to low-frequency mode initial conditions, the practical thresholds refer to observable timescales.

For nonlinear structures on chains having "weak spring" potentials, for which the nonlinear restoring force substracts from the linear restoring, the interaction that causes the final decay is radiation from the breathers to the propagating linear modes. For "strong spring" potentials the breather frequency is above the optical band, so a more subtle energy interchange must occur $[84,87,95,96]$. A beat phenomenon has been postulated as the energy interchange mechanism, and used to calculate an $\varepsilon$-scaling that agrees with numerics [92]. 


\subsubsection{Heat Transport in Lattice Models}

A main goal of classical kinetic theory is to provide the definition of transport coefficients through phenomenological constitutive equations. The basic hypotheses of this macroscopic theory of transport phenomena are the assumption that fluxes are proportional to thermodynamic forces and that the system evolves close to equilibrium [97]. For instance, when dealing with heat transport in a solid, one defines the thermal conductivity $\kappa$ through the Fourier law

$$
\mathbf{J}=-\kappa \nabla T,
$$

where the heat flux $\mathbf{J}$ is the amount of heat transported through the unit surface in unit time and $T(\mathbf{x}, t)$ is the local temperature. Such a phenomenological relation was first proposed in 1808 by Fourier as an attempt to explain the phenomenon of the Earth cooling. Equation (2.10) is assumed to be valid close to equilibrium. Actually, the very definition of the local energy flux $\mathbf{J}(\mathbf{x}, t)$ and temperature field $T(\mathbf{x}, t)$ relies, in turn, on the local equilibrium hypothesis, i.e. on the possibility of defining a local temperature for a macroscopically small but microscopically large volume in position $\mathbf{x}$ at time $t$.

The first and most elementary attempt to give a microscopic foundation to Fourier's law dates back to Debye [98]. By rephrasing the results of the kinetic theory for the (dilute) phonon gas, he found that the thermal conductivity should be proportional to $C v \ell$, where $C$ is the heat capacity and $v, \ell$ are the phonon mean velocity and free path, respectively. Moreover, Debye also realized that at a microscopic level the finite thermal conductivity in crystals should be a consequence of the nonlinear forces acting among the constituent atoms [98].

Peierls further extended the conjecture of Debye and formulated a Boltzmann-like equation, which shows that anharmonicity is necessary for obtaining genuine diffusion of the energy by the so-called Umklapp processes, where the nonlinearity is introduced phenomenologically in the transport equation, independently of the microscopic nature of the interactions [99]. Nonetheless, the Boltzmann-Peierls approach represented an improvement in the theory of lattice thermal conductivity. It allows one to compute the dependence of $\kappa$ on the temperature which agrees reasonably well with experimental data in the very low-temperature regime. However, basic questions remained, such as under which conditions is local equilibrium obtained in a physically accessible time? This kind of a problem partly inspired the numerical experiment by Fermi, Pasta and Ulam, as Fermi was aware of the conceptual difficulties concerning the possibility of constructing a satisfactory microscopic approach to transport theory. In nonlinear chains, the complex interactions among the constituent atoms or molecules of a real solid are reduced to harmonic and nonlinear springs, acting between nearest-neighbor equal-mass particles. Despite such simplifications, the basic ingredients that one reasonably conjectures to be responsible for the main physical effect (i.e. the finiteness of thermal conductivity) are contained in the model. As already 
described, the original study expected to verify a common belief, which had never been put to a rigorous test: an isolated mechanical system with many degrees of freedom should eventually yield equilibrium through "thermalization" of the energy. Furthermore, the measurement of the time interval needed to approach the equilibrium state, i.e. the "relaxation time" of the chain of oscillators, would have provided an indirect determination of thermal conductivity $\kappa$, since Debye's argument predicts $\kappa \propto C_{v} / \tau_{r}$, i.e. inversely proportional to the relaxation time $\tau_{r} \sim \ell / v$, which is assumed to represent the average time needed for a phononic excitation to relax to thermal equilibrium.

After the lack of success of the FPU numerical experiment, the first important attempt to reconsider the problem of heat transport in solids from a theoretical point of view was to consider a homogeneous harmonic chain with fixed boundary conditions in contact with stochastic Langevin heat baths [100]. The equations of motion

$$
\ddot{q}_{n}=\omega^{2}\left(q_{n+1}-2 q_{n}+q_{n-1}\right)+\delta_{n 1}\left(\xi_{+}-\lambda \dot{q}_{1}\right)+\delta_{n N}\left(\xi_{-}-\lambda \dot{q}_{N}\right),
$$

where $\xi_{ \pm}$are independent stochastic processes with zero mean and variance $2 \lambda_{ \pm} k_{\mathrm{B}} T_{\alpha}$, with $T_{+}>T_{-}$, can be solved by a phase-space description, i.e. using the Fokker-Planck equation. However, the solutions were not successful in reproducing the Fourier law. They predicted that the heat flux was proportional to the temperature difference, rather than the temperature gradient, thus showing that homogeneous harmonic chains do not exhibit normal transport properties. Although there are many aspects of linear chains, such as the inclusion of disorder or varying masses [101] that we have not considered above, our main concern here is with nonlinear chains. Numerical studies of heat conductivity in the FPU chain were reconsidered at the end of the 1960s. In particular, nonequilibrium simulations of the FPU model (2.1) with coupling constants $\alpha$ and $\beta$ fixed to represent the leading terms of the expansion of the Lennard-Jones potential were performed [102, 103]. These authors also considered the effect of disorder by including in the model either a disordered binary mixture of masses [102] or random nonlinear coupling constants [103]. The combination of nonlinearity and disorder did not help the researchers to obtain a clear understanding of the problem. They even found cases in which anharmonicity increases thermal conductivity. The attention was mainly focused on the form of the temperaure profile $T(x)$. They noticed that its shape depended on the existence of disorder. Although it is known that $T(x)$ is not a self-averaging observable for disordered harmonic chains, it is not known how $T(x)$ depends on disorder over long enough time-scales in anharmonic chains. Additional questions that were investigated concerned the concentration of impurities [102].

Preliminary work on homogeneous anharmonic chains considered the equal-masses FPU and Lennard-Jones chains composed of 30 particles and coupled with Langevin baths at their boundaries [104], a task that was unfeasible with the computer resources available at that time. As a consequence, several attempts of designing easy-to-simulate toy models followed these first 
studies. Some examples are reviewed in [105]. One was the so-called ding-aling model, a prototype of all models with an on-site potential, as described in (2.16) in the next Section. This model was found to exhibit normal thermal conductivity. The increase in computer power led to a revival of the heat conduction problem inbetween the mid-1980s and the mid-1990s, when nonequilibrium simulations of the FPU model [106, 107] and of the diatomic Toda chain $[108,109,110,111]$ of alternating light and heavy masses were performed. Subsenquently, there were systematic studies on the size dependence of the heat conductivity for the FPU chain with quartic $[112,113,114]$ or cubic [115] nonlinear potential as well as for the diatomic Toda chain $[116,117]$. They indicated a divergence of the heat conductivity with $N$, the number of mass points, which was interpreted as due to ballistic transport of energy through the chain. As we will comment in the following, an on-site potential determines a classical conductivity.

\subsection{Formulations: Types of Oscillator Chains}

\subsubsection{Chains Similar to the FPU}

Over the years, since the first numerical investigation by Fermi, Pasta and Ulam, many different oscillator chains have been studied. There have been various reasons for the particular choices, sometimes because they approximated physical systems, sometimes for their simplicity, and sometimes designed to bring out specific features or compare results with other chains.

In choosing an oscillation chain for the initial study, the FPU- $\beta$ system was a reasonable choice, as it is a discretization of the partial differential equation for the nonlinear string with a strong nonlinear restoring force

$$
\frac{\partial^{2} y}{\partial t^{2}}-\frac{\partial^{2} y}{\partial x^{2}}\left[1+3 \beta\left(\frac{\partial y}{\partial x}\right)^{2}\right]=0 .
$$

The discretization of $y(x, t)$ as $y_{j}(t)$,

$$
\begin{aligned}
\frac{\partial y}{\partial x} & =\frac{y_{j+1}-y_{j}}{\Delta x} \quad \text { or } \quad \frac{y_{j}-y_{j-1}}{\Delta x}, \\
\frac{\partial^{2} y}{\partial x^{2}} & =\frac{y_{j+1}-2 y_{j}+y_{j-1}}{\Delta x^{2}}
\end{aligned}
$$

where $\Delta x=L / N$, with $L$ as the length of the string and $N-1$ the number of oscillators, yields

$$
\begin{aligned}
& \ddot{y}_{j}=\frac{\left(y_{j+1}-2 y_{j}+y_{j-1}\right)}{\Delta x^{2}} \\
& \times\left\{(1+\beta)\left[\frac{\left(y_{j+1}-y_{j}\right)^{2}}{\Delta x^{2}}+\frac{\left(y_{j}-y_{j-1}\right)^{2}}{\Delta x^{2}}+\frac{\left(y_{j+1}-y_{j}\right)\left(y_{j}-y_{j-1}\right)}{\Delta x^{2}}\right]\right\}
\end{aligned}
$$


The original work, and also the analytic investigation by Izrailev and Chirikov [47], were with fixed end points at $j=0$ corresponding to $x=0$, and $j=N$ corresponding to $x=L$, such that $j=1,2 \ldots, N-1$ for the moving oscillators. The coordinates can be rescaled at fixed $N$ to any $\Delta x$ and $L$ to give the FPU- $\beta$ part of (2.1), with $\Delta x$ normalized to 1 . Letting $\Delta x \rightarrow \Delta x^{\prime}=L^{\prime} / L$ and introducing the change of variables $y_{j}^{\prime} \rightarrow y_{j} L^{\prime} / L$ and $t^{\prime} \rightarrow t L^{\prime} / L$ leads to (2.15) again. Since $\mathrm{d} y_{j}^{\prime} / \mathrm{d} t^{\prime}=\mathrm{d} y_{j} / \mathrm{d} t$, the energy per mode is unchanged. Thus increasing $N$ by adding oscillators to the end of the chain at fixed $\Delta x$ is equivalent to adding oscillators by subdividing the chain at fixed $L$, provided the time and displacement are rescaled.

The addition of the $\alpha$ term to (2.1) is a logical extension to a more general restoring force. However the $\alpha$ and $\beta$ terms have different properties, with the energy scaling differently with choices of $\alpha$ and $\beta$, such that the energy $E$ is renormalizable with $\beta E$ and with $\alpha^{2} E$. Furthermore, the sign in the nonlinear term in (2.12) changes the behavior from a strong to a weak spring, while the $\alpha$ term has a directional antisymmetry. Some of the consequences of these differences will emerge in the following sections.

It also became clear in subsequent years that, while fixed endpoints were a physical condition for an actual string, periodically continued endpoints (or mass points on a circle) had some attractive features for analysis. With a periodic boundary condition $(\mathrm{BC})$, waves traveling in a single direction without reflection are allowed, which is a key ingredient in the development of soliton theory, as we outline in Sect. 2.4.3. For a periodic BC, linear momentum is an exact invariant, which simplifies various analyses. If the oscillator dynamics is expressed in terms of linear modes, i.e. the modes which would be exact solutions in the absence of the nonlinearity, other differences between fixed and periodic boundaries become evident. For a periodic BC there is a highest frequency boundary mode that has exact alternation of oscillator phases, which is an exact solution of the nonlinear problem, as considered in subsequent sections.

The FPU type of oscillator chains did not realistically represent the dynamics of solid materials. A more general representation is given by the Hamiltonian

$$
H=\sum_{i}\left[\frac{p_{i}^{2}}{2 m}+U\left(q_{i}\right)+V\left(q_{i+1}-q_{i}\right)\right]
$$

where $U$ and $V$ are on-site and inter-site potentials, respectively, which are most generally nonlinear. They can be constructed as physical models of onedimensional crystals or by discretizations of Klein-Gordon partial differential equations. For the FPU chain $U=0$. One form of (2.16) that is used to compare to FPU- $\beta$ dynamics is the $\phi^{4}$ chain with $V=(1 / 2)\left(q_{i+1}-q_{i}\right)^{2}$ and $U=\left(m^{2} / 2\right) q_{i}^{2}+(\beta / 4) q_{i}^{4}$, as given in (2.9), and compared theoretically and numerically with the FPU in various subsequent sections. The Hamiltonian of (2.16) and the simplified form (2.9) are not rescalable as is the FPU- $\beta$, but the coefficients can be chosen to make useful comparisons. 
An interesting special case of the Klein-Gordon class of partial differential equations is the sine-Gordon equation

$$
y_{t}-y_{x x}+\sin y=0
$$

which can discretized in space in the same manner as the nonlinear spring to obtain the system Hamiltonian

$$
H=\sum_{i=1}^{N} \frac{1}{2} p_{i}^{2}+\sum_{i=1}^{N}\left(1-\cos y_{i}\right)+\sum_{i, j=1}^{N} A_{i j} y_{i} y_{j},
$$

where the coupling matrix $A_{i j}$ is given by

$$
A_{i j}=\frac{\left(2 \delta_{i j}-\delta_{i, j-1}-\delta_{i, j+1}\right)}{(\Delta x)^{2}},
$$

$p_{i}=\dot{y}, \Delta x=L / N$, and $\delta_{i j}$ is the Kronecker $\delta$. As with the more general forms of the discretized Klein-Gordon, (2.18) is not rescalable on $\Delta x$, such that both $L$ and $N$ enter as essential parameters. The discretized system is of particular interest, as the partial differential equation is integrable, unlike the nonlinear spring, so the discretization, itself, becomes the only source of chaos. However, at low frequencies (long wavelengths) where the FPU approximates an integrable system, the transitions are similar [54]. One interesting feature in that work was an explicit discretization of time, so forming a $2 N$-dimensional symplectic map to be analyzed. The sine-Gordon on-site potential has also been used as an interaction potential to study the Fourier heat law, as we discuss in Sect. 2.7.2. For that case the potential is known as the FrenkelKontorova potential.

Closely related to the FPU chain is the chain with the same interparticle potential structure but with varying masses. To explore the question of whether a fraction of the modes, in a distinguishable mode packet, could be isolated from the modes initially containing the energy, Galgani et al. [118] considered a modified FPU- $\beta$ model, with fixed ends, described by the Hamiltonian

$$
\mathrm{H}=\sum_{i}^{N}\left[\frac{p_{i}^{2}}{2 m_{i}}+\frac{\left(q_{i+1}-q_{i}\right)^{2}}{2}+\beta \frac{\left(q_{i+1}-q_{i}\right)^{4}}{4}\right]
$$

with $m_{i}=1$ for $i$ odd and $m_{i}=m<1$ for $i$ even. The alternation of masses separated the linear mode spectrum into branches, an acoustic branch which is only slightly modified from the usual spectrum, and an optical branch associated with the lower mass particles. The form of the spectrum is given in Sect. 2.2.2 and the implication for mode isolation in the thermodynamic limit is discussed in Sect. 2.4.2

Another oscillator chain of particular importance is the Toda lattice, which generalizes the three-particle lattice, given in (2.4), to $N$ particles. The lattice, with exponential forces between particles, is generally thought of as constrained on a ring, which is equivalent to periodic BC. This discretized chain 
is exactly integrable, which makes it uninteresting in itself, but very useful for comparing to the FPU- $\alpha$ potential that can be considered to be a truncation of the Toda potential (see Sect. 2.4.3). The same comparison of the three particle Toda potential with its truncation, the Henon and Heiles potential [11], was very useful in understanding low-dimentional chaos, as we have already discussed briefly in Sect. 2.1.2. The $N$-particle Toda chain has also been used to explore the effect of alternating heavy and light masses, which is not integrable, and is discussed briefly in Sect. 2.7.1 in connection with heat conduction.

The oscillator chains described above do not exhaust the useful types that have been explored in a variety of contexts. One such chain of historical importance is the "ding-a-ling" model consisting of alternately harmonically bound and free hard-core particles, which was used to obtain the Fourier law of heat conduction [119]. It is given in (2.132), Sect. 2.7.2, and its properties are discussed there. Variants of the ding-a-ling model have also been studied in this context [120]. The less artificial potentials of the Klein-Gordon type also can produce the Fourier law, and are considered in Sect. 2.7.2.

\subsubsection{Representation in Modes of the Linear System}

We have already mentioned that, for linear chains, the transformation to the harmonic normal modes, as given by (2.2), gives a set of mode amplitudes $Q_{k}$ that are invariant under the motion. This can be seen by applying the inverse transform, with $N$ moving particles, to the FPU- $\beta$ chain $(2.1)$ or the $\phi^{4}$ chain (2.9)

$$
q_{i}=\sqrt{\frac{2}{N+1}} \sum_{k=1}^{N} Q_{k} \sin \left(\frac{\mathrm{i} k \pi}{N+1}\right)
$$

to obtain $[121]$

$$
H_{\beta}=\sum_{k=1}^{N} \frac{1}{2}\left(P_{k}^{2}+\omega_{k}^{2} Q_{k}^{2}\right)+\frac{\beta}{8 N+8} \sum_{i, j, k, l=1}^{N} C(i, j, k, l) Q_{i} Q_{j} Q_{k} Q_{l},
$$

where $P_{k}$ are the corresponding momenta, with the mode frequencies $\omega_{k}$ given by

$$
\omega_{k}=2 \sin \left(\frac{\pi k}{2 N+2}\right)
$$

for the FPU- $\beta$, and

$$
\omega_{k}=\sqrt{m^{2}+4 \sin ^{2}\left(\frac{\pi k}{2 N+2}\right)}
$$

for the $\phi^{4}$. It is immediately apparent from Hamilton's equations that the dynamics of the linear modes are independent of one another. The quartic terms couple the modes together, with 


$$
C(i, j, k, l)=\omega_{i} \omega_{j} \omega_{k} \omega_{l} \sum_{P} B(i+j+k+l)
$$

for the FPU- $\beta$, and, for the $\phi^{4}$,

$$
C(i, j, k, l)=\sum_{P} B(i+j+k+l) .
$$

The sum is over the eight permutations of the sign of $i, j, k, l$ and the function $B(x)$ takes the value 1 if the argument is zero, -1 if the argument is $\pm 2(N+1)$, and zero otherwise. The selection rule for the couplings, which simplifies the analysis, follows from the quartic nature of the coupling (e.g., see [122]).

From (2.23) we see that frequencies spacings follow a simple sine function: they are linearly spaced (i.e. proportional to $k / N$ ) for $k \ll N$ and accumulate quadratically (i.e. as $\left.(\pi k / 2(N+1))^{2}\right)$ near the highest frequency, which lies just below 2 . For periodic boundary conditions, the frequencies are

$$
\omega_{k}=2 \sin (\pi k / N)
$$

which has only $N / 2$ different linear frequencies, and an exact zone-boundary mode with $\omega_{N / 2}=2$. The existence of this mode with exact alternation of the phase of neighboring oscillators allows one to obtain some exact solutions, which we consider in Sect. 2.4.4. For the $\phi^{4}$ chain, the linear part of the on-site potential results in the $m^{2}$ term in $(2.24)$. If $m^{2} \gg \pi k / N$, then there is also quadratic accumulation of frequencies above $\omega_{k}=m$. This bunching plays a significant role in the chaotic numerics, as described in Sect. (2.5). The FPU- $\alpha$ Hamiltonian can also be transformed by using the harmonic normal modes to obtain the transformed Hamiltonian

$$
H_{\alpha}=\sum_{k=1}^{N} \frac{1}{2}\left(P_{k}^{2}+\omega_{k}^{2} Q_{k}^{2}\right)+\frac{\alpha}{2 \sqrt{N+1}} \sum_{k, j, l=1}^{N} C(k, j, l) Q_{j} Q_{k} Q_{l},
$$

which is simpler, having only a product of three summations to represent the cubic term. Furthermore, it is considerably more stable than the FPU- $\beta$ as it is a truncation to cubic order of an $N$-particle Toda lattice, as we have considered in the previous subsection.

For numerical integrations if, for example, a single mode initial condition is used, usually with all the energy in the form of potential energy, then $E=(1 / 2) \omega_{k}^{2} Q_{k}^{2}\left(\varepsilon=\omega_{k}^{2} Q_{k}^{2} /(2 N)\right)$, and the oscillator equations are integrated with their initial values given from (2.21). Due to the nonlinearity, the energy does not remain in the initial mode but spreads through the mode spectrum, defined in terms of the instantaneous $q_{i}$ by the transformation in (2.2). For energy sufficiently low that there is no resonance overlap (see Sect. 2.4.1), the energy is principally confined to the initial mode falling exponentially to other $k$-values, but satisfying the selection rule as given by $(2.25)$ and following. Using perturbation theory, DeLuca et al. [55] obtained the mode energy decay in geometric progression 


$$
E_{h} \simeq \rho^{2} E_{h-2 \gamma},
$$

with $\gamma$ as the initial mode and $h$ the index of any high-frequency mode, and $\rho$ is the average decay ratio between modes, $2 \gamma$ apart, given by

$$
\rho=\frac{3 \beta E}{4 \pi \gamma} .
$$

The formula only holds for $\beta E \ll 1$, where resonances do not play a significant role. Numerical results for $\gamma=3$ and 5 agreed quite well with the analytical predictions of (2.29) and (2.30).

The related problem of the FPU with alternating masses, as given in (2.20), has linear normal modes with frequencies

$$
\omega_{j}=\frac{1+m \pm \sqrt{1+m^{2}+2 m \cos k_{j}}}{m}
$$

where $k_{j}=2 j \pi /(N+1)$ and $1 \leq j \leq N / 2$ (for notational convenience we use $j$ as the mode number). The acoustic branch has $0<j<N / 2$ and the optical branch for $N / 2<j<N$. The dispersion, calculated from (2.31), shows an optical branch that moves to higher frequencies and flattens as $m$ is decreased; for example, the frequency separation of the minimum optical and maximum acoustic frequency is $\Delta \omega=\sqrt{2}(\sqrt{(1 / m)}-1)$, while the optical frequency spread is $\delta \omega_{h}=\sqrt{2 / m}(\sqrt{1+m}-1) \approx \sqrt{m / 2}, m \ll 1$. The implication for isolated modes in the thermodynamic limit is discussed in Sect. 2.4.2.

Depending on the nonlinear forces, there are implications for the stability of nonlinear structures for the various forms of the linear modes. A weak spring $(\beta<0)$ in the FPU puts the nonlinear solution in the acoustic band which can then radiatively couple to the linear modes, destroying nonlinear stability. Similarly, for a strong spring $(\beta>0)$ but with an optical branch, a nonlinear acoustic mode can be shifted into the optical branch where it can dissipate by loosing energy to that branch. The various treatments of these phenomena are a major area for study (see, e.g., [84]), beyond the subject matter of this review. However, the phenomena will reappear in various subsequent sections.

\subsection{Formulations: Methods of Numerical Analysis}

Apart from the exception of integrable cases, most of the models of oscillator chains introduced in Sect. 2.2 require numerical investigation. The choice of suitable observables is then crucial to point out features of mathematical and physical interest. In this section, we introduce the description of indicators concerning both dynamical and statistical properties. In general, they are inspired by generalizations or extensions of the thermodynamic concept of entropy. 


\subsubsection{Measurement of Chaos Indicators}

A quantitative characterization of the oscillator chains is their degree of chaoticity. This is measured by the largest Lyapunov exponent, whose positivity can be a hint to the possible equipartition of the energy among the degrees of freedom. Let us briefly recall that if

$$
\dot{x}^{i}=X^{i}\left(x^{1} \ldots x^{2 N}\right)
$$

is a generic dynamical system, the tangent dynamics to this flow is described by

$$
\frac{\mathrm{d} \xi^{i}}{\mathrm{~d} t}=J_{i k}(x(t)) \xi^{k}
$$

where $J_{i k}=\partial X^{i} / \partial x^{k}$, and the largest Lyapunov exponent is given by

$$
\lambda=\lim _{t \rightarrow \infty} \frac{1}{t} \ln \frac{\|\xi(t)\|}{\|\xi(0)\|}
$$

for almost all choices of $\xi(0)$, under rather general assumptions. If $x=$ $\left(q^{1}, \ldots, q^{N}, p^{1}, \ldots, p^{N}\right)$, with $X^{i}=\left(\partial H / \partial p^{i}\right)$ for $i=1, \ldots, N$ and $X^{i}=$ $-\left(\partial H / \partial q^{i}\right)$ for $i=N+1, \ldots, 2 N$ the dynamical system (2.32) represents a Hamiltonian flow. The corresponding tangent vector is $\xi=\left(\xi^{1}, \ldots, \xi^{2 N}\right) \equiv$ $\left(\xi_{q}^{1}, \ldots, \xi_{q}^{N}, \xi_{p}^{N+1}, \ldots, \xi_{p}^{2 N}\right)$, and, by setting $\Lambda[x(t), \xi(t)] \equiv\left\{\xi^{\mathrm{T}} \underline{J}[x(t)] \xi+\right.$ $\left.\xi^{\mathrm{T}} \underline{J}^{\mathrm{T}}[x(t)] \xi\right\} / 2 \xi^{\mathrm{T}} \xi=\left[\xi^{\mathrm{T}} \dot{\xi}+\dot{\xi}^{\mathrm{T}} \xi\right] / 2 \xi^{\mathrm{T}} \xi=(\mathrm{d} / \mathrm{d} t) \ln \left(\xi^{\mathrm{T}} \xi\right)^{1 / 2} \equiv \frac{d}{d t} \ln \|\xi\|$, this can be formally expressed as a time average

$$
\lambda=\lim _{t \rightarrow \infty} \frac{1}{t} \int_{0}^{t} \mathrm{~d} \tau \Lambda[x(\tau), \xi(\tau)] .
$$

Now we want to specify the more general concept of Kolmogorov-Sinai entropy $[23,24]$ associated with the Lyapunov exponents and discuss its relevance. Besides the largest Lyapunov exponent $\lambda$, in a dynamical system made of $N$ degrees of freedom, each one described by a pair of canonical coordinates (position and momentum) one can define a spectrum of Lyapunov exponents, $\lambda_{i}$, where the index $i=1, \cdots, 2 N$ labels the exponents from the largest to the smallest one. An effective algorithmic procedure for evaluating the spectrum of Lyapunov exponents is discussed in [152]. Beyond rigorous mathematical definitions, an interpretation of the Lyapunov spectrum can be obtained by considering that the partial sum $h_{n}=\sum_{i=1}^{n} \lambda_{i}(n \leq 2 N)$ measures the average exponential rates of expansion, or contraction, of a generic volume of geometric dimension $n$ in phase space. Accordingly, $h_{1}=\lambda_{1} \equiv \lambda$ is equivalent to the definition given in (2.34), since a "one-dimensional volume" is a generic tangent segment in phase space; $h_{2}=\lambda_{1}+\lambda_{2}$ gives the divergence rate of a surface; $h_{2 N}=\sum_{i=1}^{2 N} \lambda_{i}$ is the average divergence rate of the whole phase space. In Hamiltonian systems, according to Liouville's theorem, any volume 
in phase space is conserved and $h_{2 N}=0$. Moreover, for each $\lambda_{i}>0$ there exists $\lambda_{2 N-i+1}=-\lambda_{i} \cdot{ }^{1}$ Chaotic evolution implies that a small region in phase space (for instance, the volume identifying the uncertainity region around an initial condition) is expanded and contracted with exponential rates along different directions in phase space. After a time of the order $1 / \lambda$ the distance between two infinitesimally close initial conditions will have the size of the accessible phase space; accordingly, we have no means of predicting where the image of an initial point is in phase space by knowing the image of an initially close-by point, even if after a long time these points will eventually come again close to each other (for a detailed discussion see [123]). A very important conceptual achievement is that the mechanical description of a chaotic evolution can be replaced by a description in terms of a probability distribution on phase space which is invariant under time evolution and which allows one to define a metric entropy $h$. The mathematical details go beyond the scope of this manuscript; see [123, 124, 125, 126]. For our purposes, it is important to mention that Pesin later proved, under rather general assumptions, that there exist a remarkable relation between Kolmogorov's metric entropy and the positive component of the Lyapunov spectrum [127]:

$$
h=\sum_{j, s . t . \lambda_{j}>0} \lambda_{j},
$$

where the sum extends over all the positive Lyapunov exponents. This formula can be applied to the study of the dynamics of Hamiltonian systems, like the FPU chain. In this respect, it is particularly interesting to check this formula in the thermodynamic limit, in which the number of oscillators tends to infinity. In general, this limit does not commute with the limit $t \rightarrow \infty$ in (2.5), i.e. the measurement of $\lambda$ and $h$ may depend on the order in which these limits are performed. Numerical evidence of the existence of a limit curve for the spectrum of Lyapunov exponents in the thermodynamic limit for the FPU chain was later obtained ([128]; see also Fig. 2.2 ). Further numerical evidence of the existence of such a limit for a variety of physical systems have been subsequently obtained. However, a rigorous mathematical proof is still lacking, although some attempts in this direction exist [129, 130]. The value of $h$ is expected to depend on some typical parameters, like the energy density $\varepsilon$ for a Hamiltonian chain of oscillators. For instance, the Lyapunov spectrum of the FPU- $\beta$ model shown in Fig. 2.2 is obtained for $\beta \varepsilon=10$, which is sufficiently large to yield a strongly chaotic dynamics. By decreasing $\varepsilon$ sufficiently to enter the almost-recurrent dynamical regime observed by

\footnotetext{
${ }^{1}$ For each conserved quantity like the energy, momentum etc., there is a pair of conjugated exponents that are zero. Stated differently, each conservation law amounts to a geometrical constraint that limits the access of the trajectory to a submanifold of phase space. Integrability has the consequence that all $\lambda_{i}$ are zero, i.e. there can be as many conservation laws as the number of degrees of freedom; the converse is in general not true.
} 


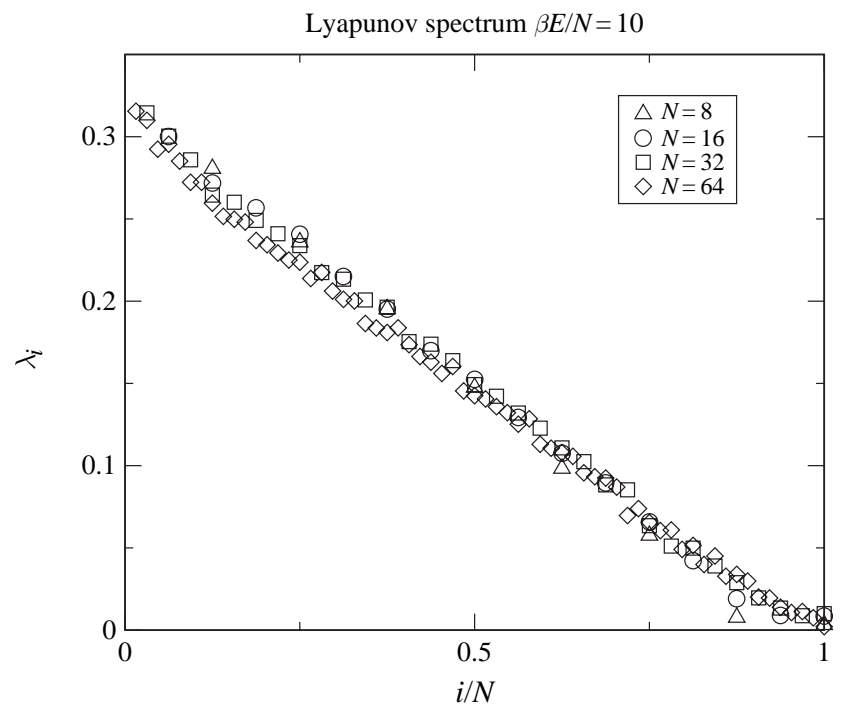

Fig. 2.2. The spectrum of positive Lyapunov exponents of the FPU- $\beta$ model for different chain lengths, from 8 up to 64 oscillators

Fermi, Pasta, and Ulam in their original numerical experiment, the shape of the spectrum also changes significantly. In this weakly chaotic regime, the maximum Lyapunov exponent is found to decrease and the positive component of the Lyapunov spectrum approaches the horizontal axis. Still the only null exponents are those corresponding to the conserved quantities, although the others take significantly smaller values and the value of $h$ is drastically reduced. According to this description, one is led to conclude that, in the thermodynamic limit, all possible chaotic degrees of freedom should remain chaotic for arbitrarily small values of $\varepsilon$, despite that beyond a certain value it will become practically impossible to distinguish them from zero. In this respect, $h$ cannot provide a characterization of the weakly chaotic regime in terms of an effective number of active degrees of freedom, as discussed in the following Section. Nonetheless, the Lyapunov analysis can provide a clear quantitative characterization of the strong and weak chaotic regimes observed in the FPU-chain. Actually, the maximum Lyapunov exponent of the FPU- $\beta$ model has been analytically estimated [131] on the basis of the geometrical approach, sketched in Sect. 2.4.7. It has been found that there is a transition value of the energy density, $\varepsilon_{\mathrm{c}}$, at which the scaling of $\lambda$ with $\varepsilon$ changes from a strong $\varepsilon$-scaling, $\lambda(\varepsilon) \propto \varepsilon^{2}$, to a weaker one $\lambda(\varepsilon) \propto \varepsilon^{1 / 4}$. The numerics is given in Sect. 2.5 and the calculation of the scaling is outlined in Sect. 2.8. This steep scaling of $\lambda(\varepsilon)$ below $\varepsilon_{\mathrm{c}}$ implies that the typical relaxation time, i.e. the inverse of $\lambda$, may become exceedingly large for very small values of $\varepsilon$. It is worth stressing that this result seems independent on the size $N$ of 
the system, thus indicating that the different relaxation regimes represent a statistically relevant effect.

\subsubsection{Equipartition Indicators: Information Entropy, Effective Number of Modes}

In the numerical experiment by Fermi, Pasta and Ulam, the initial energy was placed in a single low- $k$ mode and the authors aimed at studying how this energy would eventually flow to the other modes. The description of the dynamics in terms of Fourier modes was a natural approach at least for small specific energy, despite the fact that they are not the proper modes of the chain. They expected that the nonlinearity would yield a fast decay towards equipartition of the energy among the Fourier modes as a natural condition to be fullfilled at thermodynamic equilibrium. The existence of the two dynamical regimes in the FPU problem for low and high values of the energy density, $\varepsilon$, has been characterized in this context by introducing a suitable equipartition indicator among the Fourier modes [51, 52]. This indicator is inspired by information entropy, but, at variance with Kolmogorv's metric entropy, it relies upon a heuristic definition. In a chain made of $N$ oscillators with periodic boundary conditions there are $N / 2$ independent Fourier modes. A spectral entropy $S(t)$ can be defined as

$$
S(t)=-\sum_{n=1}^{N / 2} p_{n}(t) \ln p_{n}(t),
$$

where $p_{n}(t)=E_{n}(t) / \sum_{n} E_{n}(t), E_{n}(t)$ being the harmonic energy of the Fourier mode with wave vector $k_{n}=2 \pi n / N$ at time t. When only a single Fourier mode is excited $S(t)$ vanishes, and it takes its maximum value $S_{\max }=\ln (N / 2)$ when equipartition of the energy among the Fourier modes is obtained. Numerical studies showed that this quantity exhibits good statistical properties, while it can describe the approach to energy equipartition starting from either single-mode or multimode initial excitations. To compare chains of different lengths, a normalized quantity was defined:

$$
\eta(t)=\frac{S_{\max }-S(t)}{S_{\max }-S(0)} .
$$

Notice $\eta(t)$ tends towards zero when the system approaches equipartition and that it keeps a value close to 1 when the initial spectral entropy is maintained during time evolution. In the long time limit $\eta(t)$ was found numerically to approach an asymptotic average value $\bar{\eta}$, which was used for identifying the equipartition thresholds of the FPU- $\alpha$ and $-\beta$ models $[51,52]$. Moreover, it has been also observed that the very dynamics of $\eta(t)$ provides a qualitative characterization of the different dynamical regimes observed in these chain models [132]. The regular, quasi-recurrent dynamics of $\eta$ or of $n_{\text {eff }}=N_{\text {eff }} / N$ 
(see below) observed for small values of $\varepsilon$ turns to a fast decay towards small $\eta\left(n_{\mathrm{eff}} \sim 1\right)$ for large values of $\varepsilon$.

A more physically transparent measure is what we call the effective number of modes containing energy, which can be defined as

$$
N_{\text {eff }} \equiv \exp S
$$

which is conveniently normalized as

$$
n_{\mathrm{eff}}=N_{\mathrm{eff}} / N
$$

For oscillators, the same definitions (2.37)-(2.40) can be used, with the energy of each oscillator taken directly from the Hamiltonian, by assigning half of the difference potential to each neighbor, to obtain the normalized effective number of oscillators containing energy, $n_{\mathrm{osc}}$, which we will use in the numerics from short-wavelength mode initial conditions. The instantaneous values of $n_{\text {eff }}$ do not asymptote to one, at equipartition, due to fluctuations. A simplified calculation of the effect of fluctuations introduces a deviation $\delta e_{i}$ from equipartition $e_{i}=\bar{e}_{i}+\delta e_{i}$. Expanding the logarithmic function in $S$ in (2.37) as $\ln \left(1+\delta e_{i} / \bar{e}_{i}\right)=\delta e_{i} / \bar{e}_{i}-(1 / 2)\left(\delta e_{i} / \bar{e}_{i}\right)^{2}$ and performing the summation over $i$ yields

$$
n_{\mathrm{eff}}=n_{\mathrm{osc}}=\frac{1}{N} \exp \left\{-N \bar{e} \ln \bar{e}-N \delta_{e^{2}}^{-} /(2 \bar{e})\right\}=\exp \left\{-N \delta_{e^{2}}^{-} /(2 \bar{e})\right\} .
$$

Taking $\bar{e}=1 / N$ and making the assumption of normal statistics, that for each normal mode $\delta e^{2}=\bar{e}^{2}$ (this is confirmed by calculations), we see that $N$ cancels giving an asymptotic value $n_{\text {eff }}=n_{\text {osc }}=\exp (-0.5)=0.61$, at equipartition, for both modes and oscillators. More accurate calculations have been made separately for modes and oscillators, including the nonlinear terms in the oscillator calculation, yielding at equipartition, ([92] Appendix D),

$$
n_{\mathrm{eff}}=0.65 \quad n_{\mathrm{osc}}=0.74 \text {. }
$$

To obtain some smoothing of the numerical values of $n_{\mathrm{eff}}(t)$ and $n_{\mathrm{osc}}(t)$, various short-time averages of these quantities have been used, yielding somewhat different values from those predicted in (2.42).

\subsection{Formulations: Analytic, Low-Energy and Short-Time Results}

\subsubsection{Transformations and Low-Dimensional Calculations}

We have seen in Sect. 2.2 that a transformation to the coordinates of harmonic normal modes decouples the modes if only linear forces are present. For small values of $\beta E$, the smaller nonlinear terms couple all of the modes together. 
Taking the FPU- $\beta$ system with energy initially placed in a long-wavelength mode, which we consider here, the selection rule for the couplings results in a geometric progression of the energy fall-off to shorter-wavelength modes [55]. The strongest interactions are therefore among neighboring modes, with the initial energy in a long-wavelength mode interchanging energy most strongly with its nearest neighbors. The resulting beat oscillations, as observed numerically in the original and much subsequent work (see Fig. 2.1), involved primarily a few modes. The predominant localization among a few modes allows a useful investigation of a reduced problem, involving some minimum number of modes. To look at "resonance overlap," a four-mode subsystem is examined, which contains two three-mode resonances. This was done for the sine-Gordon chain by Goedde et al. [54] and then in more detail for the FPU- $\beta$ chain by DeLuca et al. [55]. Summarizing the analytic method, a transformation of the four-mode Hamiltonian to action-angle variables exhibits two slow angles of the major resonances $\theta_{s}=\theta_{1}+\theta_{3}-2 \theta_{2}$ and $\theta_{\mathrm{sp}}=\theta_{2}+\theta_{4}-2 \theta_{3}$. A second transformation is performed to the new variables $\theta_{s}$ and $\theta_{\text {sp }}$ followed by employing the method of averaging over the two remaining fast angles. The resultant averaged Hamiltonian has two additional approximate constants of the motion, which are the actions related to the averaged-over angles and thus is reduced to two freedoms. The resulting Hamiltonian has the approximate form

$$
\begin{aligned}
H_{4}= & \left(\frac{\pi}{N}\right)^{2} E_{\gamma}\left[-\left(J_{s}+J_{\mathrm{sp}}\right) / 4 J_{\mathrm{c}}\right. \\
& +\frac{R}{8 J_{\mathrm{c}}^{2}}\left(3 J_{s}^{2}+3 J_{\mathrm{sp}}^{2}-4 J_{s} J_{\mathrm{sp}}+J_{\mathrm{c}} J_{s}-2 J_{\mathrm{c}} J_{\mathrm{sp}}\right) \\
& +\frac{R}{16 J_{\mathrm{c}}^{2}} \sqrt{J_{1} J_{2} J_{3} J_{4}} \cos \left(\theta_{\mathrm{sp}}+\theta_{s}\right) \\
& \left.+\frac{R}{16 J_{\mathrm{c}}^{2}}\left(\sqrt{J_{1} J_{2}^{2} J_{3}} \cos \theta_{s}+\sqrt{J_{2} J_{3}^{2} J_{4}} \cos \theta_{\mathrm{sp}}\right)\right],
\end{aligned}
$$

where $J_{c}, J_{d}, J_{s}$, and $J_{\mathrm{sp}}$ are the transformed actions and $J_{1}, J_{2}, J_{3}$, and $J_{4}$ are the original actions, related to the transformed actions by the canonical transformation

$$
\begin{aligned}
& J_{1}=J_{s} \\
& J_{2}=J_{\mathrm{sp}} \\
& J_{3}=J_{s}-2 J_{\mathrm{sp}}+J_{\mathrm{c}} \\
& J_{4}=J_{\mathrm{sp}}+J_{d} .
\end{aligned}
$$

$J_{c}$ and $J_{d}$ are new constants of the motion, resulting from the averaging, and $J_{\mathrm{c}}$ was chosen such that $J_{\mathrm{c}}=E_{\gamma} / \Omega_{\gamma}$, i.e. the action corresponding to the initial energy, primarily in mode $\gamma$. The concept of "resonance overlap" is taken from low-dimensional chaos theory, which considers separately the phase space motion $H\left(J_{s}, \theta_{s}\right)$ with $J_{\mathrm{sp}}=$ const. and $H\left(J_{\mathrm{sp}}, \theta_{\mathrm{sp}}\right)$ with $J_{s}=$ const., 
with overlap being the condition that for some values of $\theta_{s}$ and $\theta_{\mathrm{sp}}$ we obtain $J_{s}=J_{\mathrm{sp}}$. The variable actions $J_{s}$ and $J_{\mathrm{sp}}$ are numerically studied by looking at the phase space of one degree of freedom in a surface of section of the other freedom, with area filling trajectories indicating resonance overlap. The overlap is governed by

$$
R=(N+1) \frac{6 \beta}{\pi^{2}} E_{\gamma} \geq 1
$$

where $R$ measures the ratio of nonlinear to linear energy in the resonant degrees of freedom, analogous to the energy ratios used to calculate the mode overlap condition in (2.7). As in that calculation, from our understanding of low-dimensional chaos, we expect significant stochasticity to appear for $R>1$. $R=1$ has recently been shown to be the transition to instability for periodic solutions of the full chain [133].

The results for four $R$-values are given in Fig. 2.3, showing the transition to stochasticity in the reduced system. From the same four-mode calculation, the frequency of a typical resonant trajectory is given by

$$
\Omega_{\mathrm{B}} \approx \mu \gamma \beta E_{\gamma}\left(\frac{\pi}{N}\right)^{2}
$$
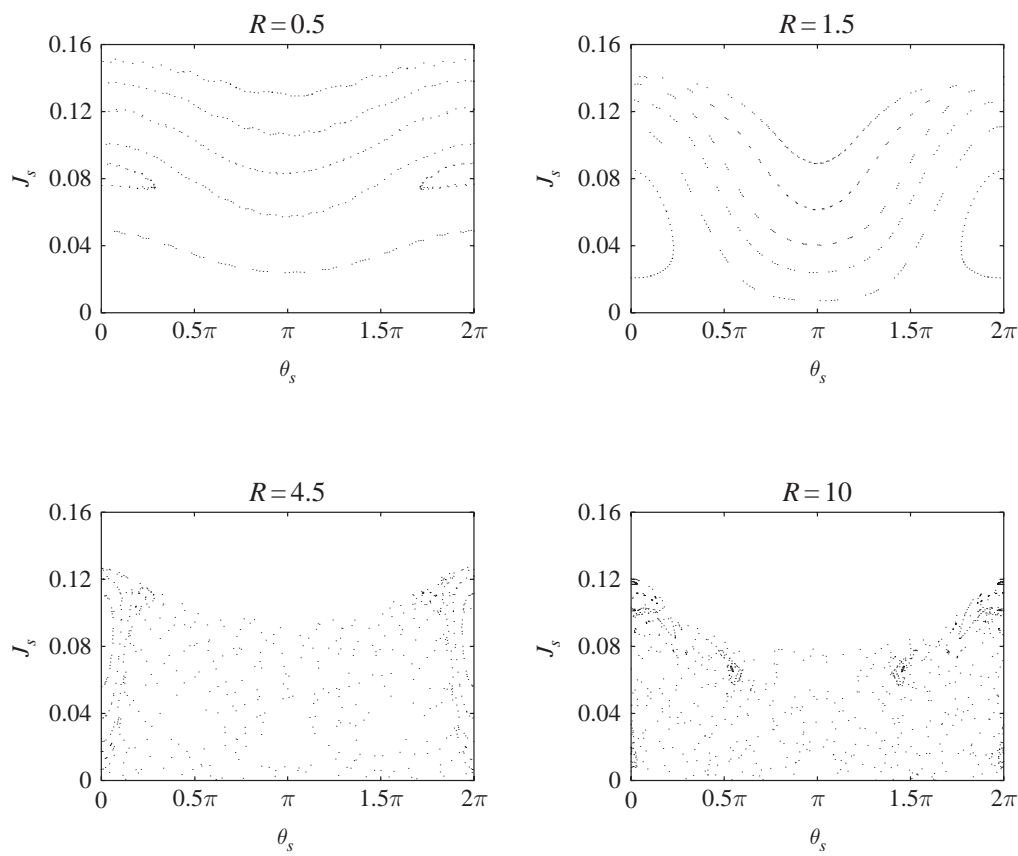

Fig. 2.3. Surfaces of section of the averaged $H_{4}$ system in formula (2.43) with two slow angles and six initial conditions per picture, we plot $J_{s}$ vs. $\theta_{s}$ 
with $\mu$ a constant of order unity, dependent on the particular initial conditions. (Here and afterward, we use the approximation $N+1 \approx N$.)

The transition to stochasticity in a reduced system is neither necessary to ultimately reach equipartition, nor sufficient to produce equipartition on numerically observable time-scales. We note from (2.48), as the number of freedoms increases overlap occurs at decreasing energy. However, from (2.49), we see that the stochasticity also exhibits itself on increasingly slow timescales. Furthermore, there is a competition between local resonance overlap, which spreads energy among neighboring modes, and the process of Arnold diffusion, which transports energy along guiding resonances to modes in other parts of the phase space. This latter process is exponentially slow at low energy. Although a rigorous upper bound on Arnold diffusion has the form given in (2.3), this does not determine the diffusion rate from the long wavelengths to the short wavelengths. The appropriate calculation is made from a three-resonance model ([13]; see also [15], Sects. 6.1 and 6.2). We have already considered the two resonances, which produce the local stochasticity. The third resonance, called the guiding resonance, links two short-wavelength modes to the low frequencies via the selection rule (2.25). Again, following [55], the calculation yields a rate of energy increase in the short-wavelength mode proportional to $\exp (-\pi / \varepsilon)$, with $\varepsilon \approx \Omega_{\mathrm{B}} / \delta \Omega_{h}$ where $\delta \Omega_{h}$ is the shortwavelength resonance frequency. Thus we expect the diffusion to be numerically observable if $\Omega_{\mathrm{B}}>\delta \Omega_{h}$, i.e. the low frequency beat becomes comparable to a high-frequency resonance that it can couple to, that is, one for which $B \neq 0$ in (2.25). The smallest $\delta \Omega_{h}$ (largest $\varepsilon$ ) is $\delta \Omega_{h}=\gamma(\pi / N)^{2}$. Substituting for $\Omega_{\mathrm{B}}$ from $(2.49)$, together with this $\delta \Omega_{h}$ yields the inequality

$$
\mu \beta E_{\gamma}>1
$$

for diffusion along resonances to compete with diffusion across resonances. Here, as in all other equations $\beta E$ appears as a product, which measures the nonlinearity. The implications of (2.50) can be seen in numerical calculations in Fig. 2.4 at small values of $R$ for some relatively small oscillator chains, for which the lower edge gives a long-time asymptotic value of $N_{\text {eff }}$. Considering that for $R>1$ there is strong local coupling among modes, then as $R$ increases and the energy interchange spreads to more modes, there is an increase of $N_{\text {eff }} \propto R$, given by this lower edge. However, at some value of $E_{\gamma}=E_{\mathrm{c}}$, satisfying (2.50) the values of $N_{\text {eff }}$ leave this asymptote, and, in fact, approach equipartition over longer times. This scaling, first found numerically in [56], is physically explained by the direct transfer of energy through the guiding resonances to high-frequency modes (see [55] for a more detailed calculation). We illustrate the spreading to higher modes in Fig. 2.5 at $R=2.9$ for $N=$ 32 , below the $E_{\mathrm{c}}$ transition as found in Fig. 2.4. The increase in energy in some high-frequency modes, specified from the selection rules, is above the background, but does not increase with time. We will contrast this result with the spectrum for $E>E_{\mathrm{c}}$ in Fig. 2.9, which approaches equipartition as time increases. 


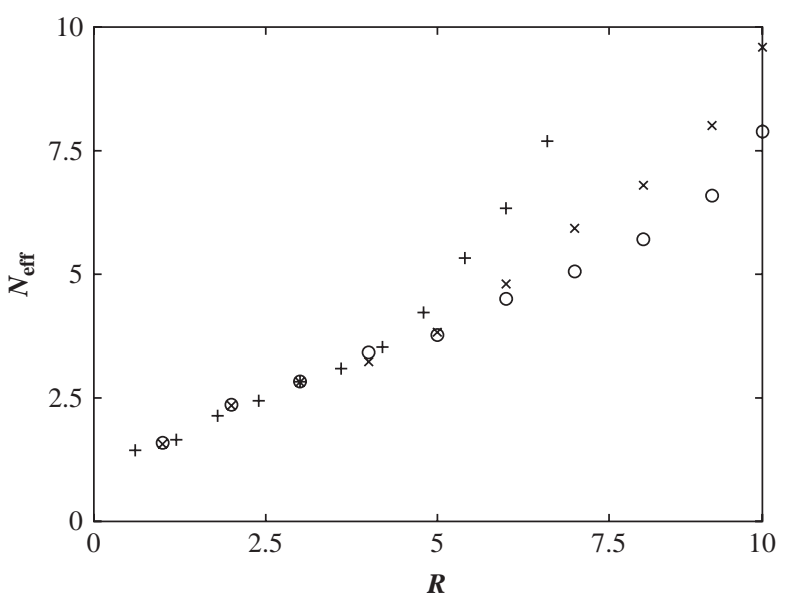

Fig. 2.4. Effective number of modes $N_{\text {eff }}$ vs. $R$ after $t=2000\left(3 /\left(\gamma(\pi / N)^{2}\right) ;\right.$ pluses $N=16 ;$ crosses $N=32$; circles $N=64$

As with the FPU- $\beta$ oscillator chain, the FPU- $\alpha$ can also be analyzed in terms of overlapping resonances to determine the onset of large stochastic layers among the long-wavelength modes. Because the nonlinear term is cubic, rather than quartic, the resonances are simpler, involving only three terms, and the scaling, with $\varepsilon$, for resonance overlap, is different. Shepelyansky [62] has used the same averaging procedure as described in the four-mode approximation of the FPU- $\beta$, to analyze the FPU- $\alpha$ chain, obtaining the Hamiltonian

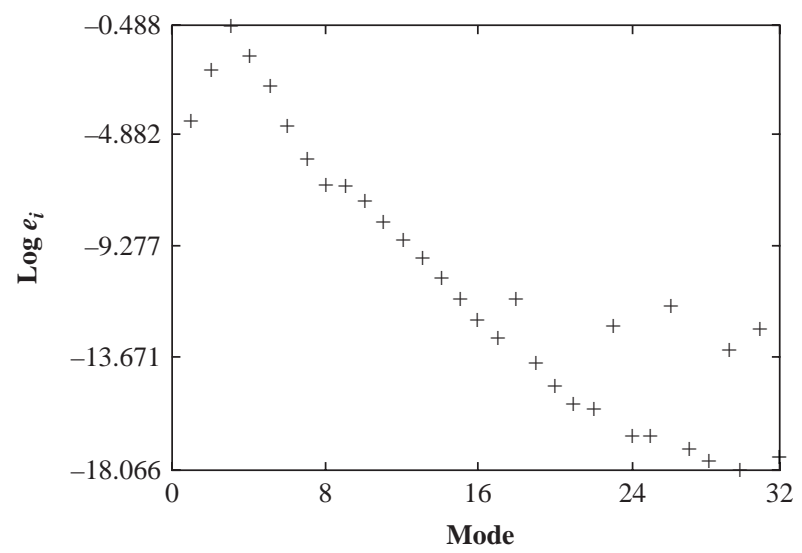

Fig. 2.5. Log of average energies at $R=2.9$ for $N=32(E=1.4)$ after $t=$ $2000(N / \pi)^{2}$ 


$$
\begin{aligned}
H= & \sum_{k=1}^{N} \omega_{k} I_{k}+\frac{\alpha}{2 \sqrt{N+1}} \cdot \sum_{k_{1}, k_{2}, k_{3}=1}^{N}\left(\omega_{k_{1}} \omega_{k_{2}} \omega_{k_{3}} I_{k_{1}} I_{k_{2}} I_{k_{3}}\right)^{1 / 2} \\
& \times \cos \left(\theta_{k_{3}}-\theta_{k_{2}}-\theta_{k_{1}}\right) \delta_{k_{3}, k_{1}+k_{2}}
\end{aligned}
$$

where all the angles have been averaged over, except the resonant ones for which $k_{3}=k_{1}+k_{2}$ in the long-wavelength spectrum. For these wavenumbers from $(2.23), \omega_{k} \approx \pi k /(N+1)$ such that $\omega_{3} \approx \omega_{1}+\omega_{2}$. The Is and $\theta$ s are the action-angle variables, as in (2.43), before the final transformation to the resonant coordinates. Because of the lower cubic products, Shepelyansky was able to examine the full Hamiltonian, and after making two simplifying further transformations he derived the approximate chaos border at long wavelengths, $\gamma \ll N$, where $\gamma$ is the $k$-value at the center of the resonance

$$
\alpha N^{3 / 2} E^{1 / 2} / \gamma^{2}>1
$$

Comparing (2.52) with (2.48), which has $R>1$ for resonance overlap, we see that the scaling with the perturbation strengths $\alpha$ or $\beta$ are the same, as is the scaling of $\varepsilon \propto N^{2}$ if we substitute for the energy density $\varepsilon=E / N$ in both cases. Thus for fixed energy density (fixed temperature), both formulas predict a resonant transition to local chaos in the thermodynamic limit, $N \rightarrow$ $\infty$. The energy-dependence with quartic or cubic nonlinearities is, of course, different. Shepelyansky investigated the transition of (2.52) numerically, using the largest Lyapunov exponent, finding reasonable agreement. He also fits the distribution of linear mode energies to the distribution

$$
E_{k} \propto \frac{1}{k_{\mathrm{c}} \exp \left(k / k_{\mathrm{c}}-c\right)+1}
$$

(with the best fit for $c=2.65$ ) such that $k_{\mathrm{c}}$ is a measure of the number of modes containing energy, similar to $N_{\text {eff }}$, but for early times for which the energy distribution still decreases exponentially with mode number, i.e. the energy has not significantly diffused to the high frequencies through the Arnold Web. The numerical estimate for the scaling is

$$
k_{\mathrm{c}} \sim\left(N^{3} \alpha^{2} E\right)^{1 / 4}
$$

which the author was able to predict analytically. This is contrasted with the result from Fig. 2.4, which indicates that

$$
N_{\text {eff }} \sim(N \beta E)^{m}
$$

i.e. is governed by the number of modes that can satisfy the local overlap condition $R>1$ with $R$ given in (2.48). Shepelyansky [62] has analytically estimated $m=1 / 2$.

The FPU- $\alpha$ model can be obtained as a third order truncation of the power series expansion of the Toda lattice potential, defined by the Hamiltonian: 


$$
H(p, q)=\sum_{k=1}^{N} \frac{p_{k}^{2}}{2}+\frac{a}{b} \sum_{k=1}^{N}\left[\exp \left(-b\left(q_{k+1}-q_{k}\right)\right)+b\left(q_{k+1}-q_{k}\right)-1\right]
$$

Since the Toda lattice is integrable, i.e. does not exhibit stochastic behavior, the FPU- $\alpha$ is more stable than the FPU- $\beta$. However, because the nonlinear potential is cubic the trajectories become unbounded at high energy. Therefore, it is restricted to examining low-energy phenomena, as was described above. Using (2.35) for calculating $\lambda$ for neighboring trajectories, and choosing the constants $a$ and $b$ in (2.56) to correspond to the FPU- $\alpha$ given in (2.1), Pettini and co-workers [61] compared the variation with time of the integrable and nonintegrable systems, with the result as shown in Fig. 2.6. The initial conditions, starting on separate orbits, separate linearly (see [15]) from which, calculating from (2.35) over short times, a large Lyapunov exponent is obtained. However this effect continually diminishes in the averaging process and, after a long time, only an average exponential divergence of the trajectories remains. In Fig. 2.6 we show the value of $\lambda$ stabilizing at the average exponent for the FPU- $\alpha$ system, while it vanishes for the Toda system. As an aside remark, we point out that the stabilized value of $\lambda$, shown in this figure, is not necessarily the asymptotic value, but may correspond to a value in a more localized region of the phase space. Without exploring this possibility in detail we note that the numerical values of $\lambda$ presented in Sect. 2.5, and compared to calculations in Sect. 2.8, have been obtained in a way that should be close to the infinite-time average.

Considerable effort has been directed toward the comparison of the FPU$\beta$ chain with oscillator chains constructed from discretization of the KleinGordon equation, particularly the $\phi^{4}$ chain, with the nonlinear term being

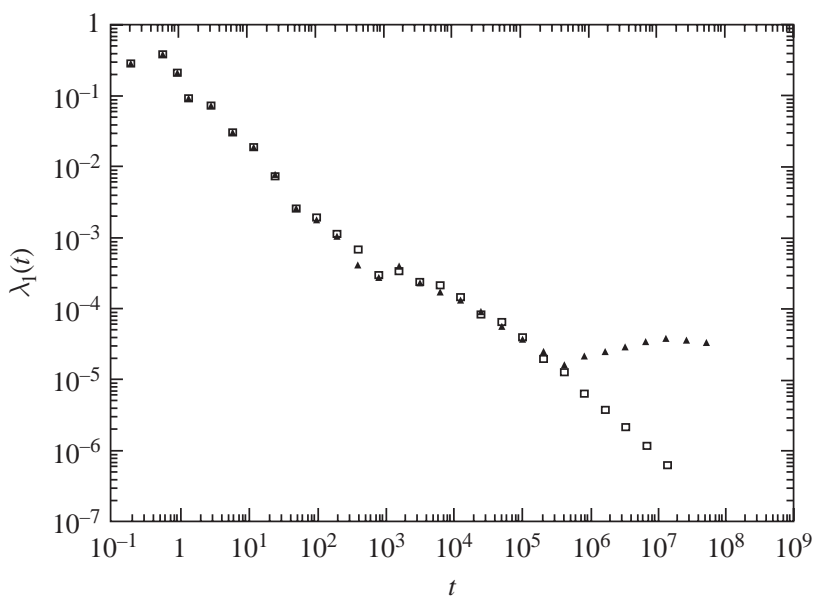

Fig. 2.6. Maximal Lyapunov exponent vs. time for the Toda lattice (open squares) and for the FPU- $\alpha$ model (solid triangles) for $N=32$ and $\varepsilon=0.0217$ 
an on-site potential. However, little attention was given to the comparison on shorter time-scales, from long-wavelength initial conditions. Comparing the coefficients in (2.25) and (2.26), for small $m$, we see that the nonlinearity is much weaker for long wavelengths (small $\omega$ for the FPU potential than for the $\phi^{4}$ potential). The opposite holds for short wavelengths, where the $\omega$ s are about 2 (for small $m$ ). Physically this is easily understood, as the forces between neighboring oscillators are quite small for the FPU at long wavelengths: neighboring oscillators are in phase, with nearly the same amplitudes, while at short wavelengths the nearly out of phase amplitudes amplifies the forces between them, as compared to the nonlinear self-force of the $\phi^{4}$. The consequences for the times to achieve equipartition, starting from either low- or high-frequency initial conditions, will be presented in Sect. 2.8. The strong nonlinearity, coupled with the weak dispersion at short wavelengths, which is evident from either (2.23) or (2.24), leads to narrow structures in the oscillator space, which exhibit the short-time characteristics of breathers. These structures called chaotic breathers (CB's) are introduced in Sect. 2.4.4, and investigated in some detail in Sects. 2.6 and 2.7.2.

\subsubsection{The Thermodynamic Limit}

The analysis described in Sect. 2.4.1 of considering a few modes which contain most of the energy, to understand the subsequent behavior, is appropriate for finite, relatively small, values of $N$. We have already seen that for fixed $\varepsilon$ and increasing $N$, (2.48), with $R>1$, for the FPU- $\beta$ and (2.52) for the FPU- $\alpha$ indicate local stochasticity and therefore diffusion throughout the phase space in the Arnold web, but without specifying the time-scale. For fixed $\varepsilon=E / N$, approaching the thermodynamic limit $(N \rightarrow \infty)$, other questions arise.

In Sect. 2.4.1 we saw, from various perspectives that at fixed $E$ the number of modes forming an energy-containing packet, in a reasonably short time, would increase with $N$, while the fraction of modes $n_{\text {eff }}=N_{\text {eff }} / N$ would remain constant. This implied that for fixed $E$ equipartition would not be reached, at least for computationally observable times, for large values of $N$. However, at fixed $E, \varepsilon$ decreases with increase in $N$, so the question of what happens for fixed $\varepsilon$ in the thermodynamic limit was not addressed.

In Sect. 2.3.1, we numerically indicate that the value of the largest Lyapunov exponent decreases with the power law $\lambda \propto \varepsilon^{2}$, but for low values of $N$ there are faster drop-offs, which may be exponentially varying, as suggested in the previous section. However, the drop-off value of $\varepsilon$ occurs at increasingly small $\varepsilon$ as $N$ is increased. Similarly, in Sect. 2.5.2, the time to equipartition $T_{\text {eq }}$ increases as a power law in $\varepsilon$ at smaller $N$, with any faster increases appearing at smaller $\varepsilon$ as $N$ is increased. The implication is that $T_{\text {eq }} \propto \varepsilon^{-3}$ at the thermodynamic limit.

From a different perspective, Galgani and coworkers [48, 49] used a convergent perturbation theory to rigorously show that two groups of oscillators, well separated in frequency space, would transfer energy exponentially slowly 
from the low to the high frequencies, i.e. energy would be "frozen out" of the high-frequency oscillators over times $\tau \propto \exp \left(a \omega_{h} / \omega_{l}\right)$ the ratio of the high to low frequencies. Using these ideas, they estimated transfer times from low to high frequencies for the FPU chain, but came to the conclusion that the energy could not be bounded away from the high frequencies for exponentially long times in the thermodynamic limit [118]. In the same paper they returned to the concept of two well-separated groups of oscillators by employing the alternating mass chain given in (2.20). As described in Sect. 2.2.2, with the lighter mass $m$ much smaller than the heavier unit mass, an optical branch becomes thin with $\delta \omega_{h} \approx(m / 2)^{1 / 2}$ and can be treated in perturbation theory as occurring at a single high frequency $\omega_{h} \approx(2 / m)^{1 / 2}$. The upper edge of the acoustic band is $\omega_{l(\max )} \sim 1$. The ratio appears in the rate of change of the high-frequency action proportional to $\exp \left(-B\left(\omega_{h} / \omega_{l(\max )}\right)\right)$ at fixed $N$ and $\varepsilon$, in qualitative agreement with their previous work. However, $B$ vanished inversely with a power of $N$, and thus the high frequency modes were not isolated in the thermodynamic limit. Their numerical studies with $0<N<200$ were not conclusive, but indicated a weak $N$-dependence.

A similar oscillator chain to that given in (2.20), but with the heavy and light masses distributed randomly, also resulted for finite $N$ in only a partial filling of the modes [134]. A calculation of $n_{\mathrm{eff}}$, starting from long-wavelength initial conditions, indicated that only the acoustic modes came to equipartion in the time-scales investigated, with very little energy in the optical branch. This is also consistent with exponentially slow transfer to the optical branch, but definitive answers in the thermodynamic limit cannot be obtained from numerically observable times. We present results in Sect. 2.5.

\subsubsection{Long-Wavelength Approximations: KdV, mKdV; Stability; Exact Periodic Solutions}

In the introduction, we described, briefly, that Taylor series expansions for long wavelengths of the FPU- $\alpha$ and FPU- $\beta$ chains result respectively in the $\mathrm{KdV}$ and $\mathrm{mKdV}$ partial differential equations. We illustrate the method for the most extensively studied case of the mKdV approximation to the FPU- $\beta$ chain. Starting from the differential form of the oscillator equations

$$
\frac{\partial^{2} y_{j}}{\partial t^{2}}=\left(y_{j+1}-y_{j}\right)-\left(y_{j}-y_{j-1}\right)+\frac{1}{3}\left[\left(y_{j+1}-y_{j}\right)^{3}-\left(y_{j}-y_{j-1}\right)^{3}\right] \text {, }
$$

where the displacements of the lattice sites have been rescaled to obtain the nonlinear coefficient 1/3, Zabusky and Kruskal [44] used a Taylor expansion to make $y$ into a continuous variable $y(x, t)$, using a integro-differential form

$$
u \equiv-\frac{y_{t}}{2 h}+\frac{1}{2} \int_{0}^{y_{x}} \sqrt{1+h^{2} \eta^{2}} \mathrm{~d} \eta
$$

to transform (2.57) in lowest order in $\Delta x \equiv h=L / N$ into the equation 


$$
u_{\tau}+12 u^{2} u_{\xi}+u_{\xi \xi \xi}=0,
$$

where $L$ is the chain length, $N$ the number of oscillators; the time and length variables have been rescaled by $\tau=h^{3} t / 24$ and $\xi=x-h t$, and the subscripts $t, x, \tau, \xi$ denote differentiation with respect to that variable.

Defining $u$ by a differential in $t$ and an integral in $x$ reduced the time derivatives by one and increased the space derivative by one to obtain the well-known mKdV equation, which is integrable as we show below. We note, however, that the choice of a unique direction in the variable $\xi$ implies a traveling solution and thus a solution only exists as a single nonlinear wave on an infinite or periodic chain.

Periodic solutions, stationary in the frame $\xi-C \tau$, can be obtained by integrating (2.59) twice, giving

$$
\frac{1}{2} u_{\xi}^{2}+u^{4}-\frac{1}{2} C u^{2}-B u+A=\frac{1}{2} u_{\xi}^{2}+P(u)=0,
$$

where $A$ and $B$ are constants of integration. For a periodic lattice the mean of $u$ must be zero which implies $B=0$. Equation (2.60) is in the form of a one-degree-of-freedom Hamiltonian, which is therefore integrable. Equilibrium solutions to (2.60) have been obtained in terms of the Jacobi elliptic functions (or cnoidial waves) $c n(\xi, q)$, with $q^{2}$ (the modulus) taken as a parameter with $0 \leq q \leq 1$. Driscoll and O'Neal [45,46] examined the solutions for stability, both analytically for long-wavelength perturbations, and numerically. They determined the unstable modes of any solution $u_{0}$ by numerically solving the linearized eigenvalue problem

$$
\mathrm{i} \nu v-C v_{\xi}+12\left(u_{0} v\right)_{\xi}+v_{\xi \xi \xi}=0,
$$

where $u(\xi, \tau)=u_{0}(\xi)+v(\xi) \exp (\mathrm{i} \nu \tau)$. The waves are found to be stable if all four roots of the associated polynomial $P(u)$ are real, and unstable if two roots are real and two are complex. Although the normalized time $\tau$ is scaled by $h \propto 1 / N$, the complete rescaling of the normalized equations back to unnormalized variables gives growth rates independent of $N$. Driscoll and O'Neil then compared the growth rates with those found from numerical integration of the equations of motion for the FPU chain with various values of $N$. For wavenumber $\pi k / L$ they obtain the growth rate as a function of the modulus $q^{2}$ of the elliptic function, as shown in Fig. 2.7. In this situation the growth of the unstable modes in the continuous limit is found to be an upper bound on the mode growth of the finite chain. A similar relation was also found to exist between the existence of instability in the sine-Gordon equation and equipartition in an oscillator chain corresponding to the discretized sine-Gordon equation [95]. For the FPU system we would not expect the mKdV instability to be directly related to equipartition among the high frequency modes, as the $\mathrm{mKdV}$ differential equation does not describe these modes. However, mixing of low-frequency modes in the continuous system corresponds to stochasticity among low-frequency modes in the discrete system. To explore this further, 


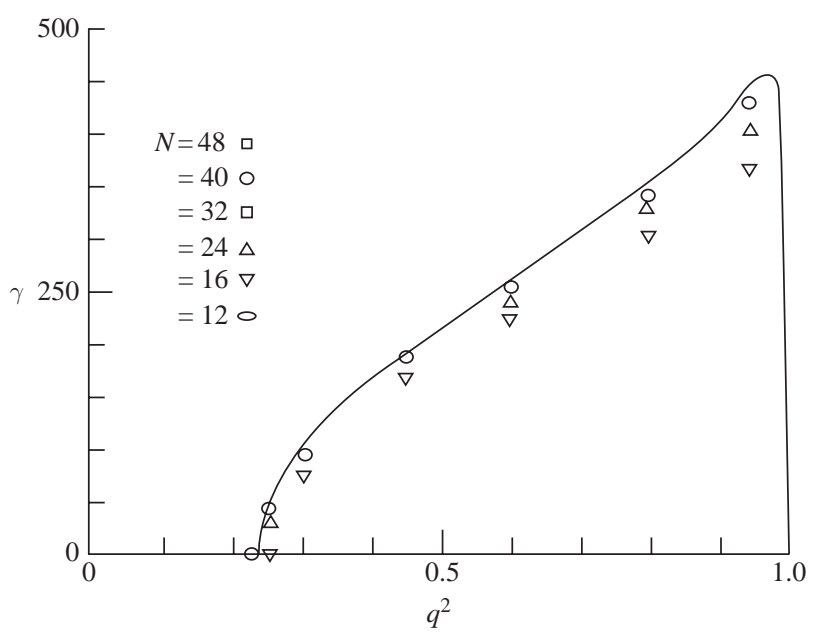

Fig. 2.7. Scaled lattice instability rate $\gamma$ (data points) and $\mathrm{mKdV}$ prediction (solid curves) for cnoidal waves vs. modulus $q^{2}$

we compare the onset of instability in the rescaled parameters of the mKdV equation, as found by Driscoll and O'Neal, to the parameters governing the interaction among the low-frequency modes in the four-mode analysis. The rescaling of $R$ from (2.48) gives the relationship $R=\left(8 / \pi^{2}\right)(\gamma / 2)^{2} q^{2} K^{2}\left(q^{2}\right)$, where $\gamma$ is the number of nodes of the cnoidal function and $K\left(q^{2}\right)$ is the complete elliptic integral. The instability appears for $q^{2} \approx 0.25(K=1.7)$ for $\gamma=2$, which corresponds to $R_{\mathrm{c}} \approx 0.58$. This is close to the value which produced a separatrix layer in the four-mode resonance interaction found in Sect. 2.4.1, so we conclude that the mechanisms are related. The single-mode initial conditions give rise to beat phenomenon, corresponding to stable solitons. It is only when the solitons become unstable that this manifests itself as chaos in the discrete system.

For the FPU- $\alpha$ oscillator chain a similar Taylor expansion and transformation to new variables, gives the $\mathrm{KdV}$ equation

$$
u_{\tau}+u u_{\xi}+\frac{1}{24} u_{\xi \xi \xi}=0
$$

which is also integrable. However, for the $\mathrm{KdV}$ equation, linearization of the solution does not exhibit unstable eigenvalues, and thus chaos appears when the Taylor expansion breaks down at larger values of energy when the discreteness leads to diffusion among the low-frequency modes. As with the mKdV equation, a single soliton, which travels in a given direction, cannot satisfy fixed boundary conditions so that more than one soliton is required to describe any prescribed initial condition.

For either the KdV or mKdV equation an initial condition consisting of a long-wavelength linear mode, say 


$$
u(\tau=0, \xi=x)=u_{0} \cos \left(\frac{2 \pi x}{L}\right),
$$

where $u \sim \varepsilon^{1 / 2}$ ( $\varepsilon$ is the energy per degree of freedom in the FPU) will break up into a set of solitons. The shortest wavelength of the resulting solitons can be estimated from dimensional analysis $[44,135]$. For long wavelengths, the dispersion is weak, therefore the dynamics is dominated by the nonlinearity. One can therefore neglect the dispersive term, which for the KdV equation (2.62) satisfies $(1 / 24) u_{\xi \xi \xi} /\left(u u_{\xi}\right)<1$ or dimensionally

$$
\frac{l^{-3}}{(\sqrt{\varepsilon} / l)} \leq 1 \text { or } l_{\min } \sim \varepsilon^{-1 / 4} .
$$

By reintroducing $\alpha$, using the scaling $\varepsilon \rightarrow \alpha^{2} \varepsilon$, and by considering that the fraction of degrees of freedom $n_{\text {eff }} \simeq 1 / l_{\text {min }}$, we obtain

$$
n_{\mathrm{eff}} \sim \alpha^{1 / 2} \varepsilon^{1 / 4} .
$$

This result agrees with the scaling estimated and found numerically by Shepelyansky [62], if $n_{\mathrm{eff}}=N_{\mathrm{eff}} / N$ is substituted in (2.54) and also coincides with later studies of Biello et al. [136] and Berchialla et al. [137]. In a manner similar to that described above, the scaling of $n_{\text {eff }}$ with $\beta \varepsilon$, for the FPU- $\beta$, can be determined from the ratio of dispersive to nonlinear terms in (2.59), giving

$$
\frac{l^{-3}}{\varepsilon / l} \geq 1 \text { or } l_{\min } \sim \varepsilon^{-1 / 2} .
$$

Changing $\varepsilon$ to $\varepsilon \rightarrow \beta \varepsilon$ and $n_{\text {eff }} \simeq 1 / l_{\text {min }}$, we obtain

$$
n_{\mathrm{eff}} \sim(\beta \varepsilon)^{1 / 2} .
$$

This result is in agreement with the estimate by Shepelyansky [62], but different from the scaling found numerically in Fig. 2.4, which, however, has not been examined for large $N$, where (2.67) applies.

It has also been recently shown [133] that it is possible to construct exact periodic solutions of the FPU chains, for fixed $\beta \varepsilon$ at finite $N$, by a Newton method. The authors called the solutions $q$-breathers (QBs) in analogy to short-wavelength solutions of a few oscillators, since they designated the linear mode number by $q$. These solutions are complementary to those obtained from continuous approximations. For the FPU- $\alpha$ and FPU- $\beta$, asymptotic expansions in the small parameters $\rho=\alpha / \sqrt{2(N+1)}$ and $\sigma=\beta /(2(N+1))$, respectively, produce exponentially decaying linear-mode spectra, similar to those described in Sect. 2.4.1, and similar to expansions obtained from soliton solutions with periodic boundary conditions. Unlike the solitons, the periodic solutions cannot be summed to produce the initial conditions of a single linear mode. Nevertheless, for small perturbation parameters, they are sufficiently close to single-mode initial conditions that interesting results can be obtained 
from them to compare to the usual numerics. For the FPU- $\beta$, the periodic solutions become unstable, similar to the instability we have seen in the $\mathrm{mKdV}$ equation. A very interesting result is that the bifurcation to unstable solutions of the FPU- $\beta$ chain occurs at a value of $R=1+\mathrm{O}\left(1 / N^{2}\right)$; from Sect. 2.4.1 we recall that $R=6 \beta E(N+1) / \pi^{2}$ relates nonlinear to linear terms in the Hamiltonian. The condition $R \simeq 1$ was a semi-quantitative transition for resonance overlap, leading to local chaos. Here we find that the condition becomes precise for the onset of an instability that also leads to local chaos. We note again, as in Sect. 2.4.1, that with increasing $N$ the value of $\beta E$ at which the instability occurs continually shrinks to reach zero in the thermodynamic limit.

\subsubsection{Short-Wavelength (high-frequency) Initial Conditions}

Let us consider the equations of motion in Fourier space for the FPU- $\beta$ model with periodic boundary conditions $q_{i}=q_{i+N}$

$$
\ddot{Q}_{r}=F_{r}\left(Q_{1}, \ldots, Q_{N-1}\right), \quad r=1, \ldots, N-1,
$$

where

$$
F_{r}\left(Q_{1}, \ldots, Q_{N-1}\right)=-\omega_{r}^{2} Q_{r}-\frac{\beta \omega_{r}}{2 N} \sum_{j, k, l=1}^{N-1} \omega_{j} \omega_{k} \omega_{l} C_{r j k l} Q_{j} Q_{k} Q_{l}
$$

and the frequencies are in this case

$$
\omega_{k}=2 \sin \left(\frac{\pi k}{N}\right) \quad k=0, \ldots, N-1 .
$$

The coupling coefficients, analogous to those in (2.25) for fixed boundaries, are given by

$$
C_{i j k l}=-\Delta_{i+j+k+l}+\Delta_{i+j-k-l}+\Delta_{i-j+k-l}+\Delta_{i-j-k+l},
$$

where

$$
\Delta_{r}= \begin{cases}(-1)^{m} & \text { for } r=m N \text { with } m \in Z \\ 0 & \text { otherwise } .\end{cases}
$$

The center of mass motion is decoupled; this is why the sum in (2.68) extends up to $N-1$. A natural question that arises is whether a set of modes exists which is decoupled from the others. If we put the energy only in this set, this is not shared by the others. Such a set is an invariant manifold in Fourier space. The question of existence has been positively solved [70, 71]. For instance, modes

$$
k=\frac{N}{4} ; \frac{N}{3} ; \frac{N}{2} ; \frac{2 N}{3} ; \frac{3 N}{4}
$$

are decoupled and are the only one-mode solutions with this property. The time-dependence of such periodic solutions is given in [70]. Moreover periodic 
and quasi-periodic solutions evolving on two-mode manifolds have been derived, and a full classification of higher dimensional invariant manifolds has been obtained. The existence of these invariant manifolds is related to spatial symmetries $[71,72]$.

The question of linear stability is more difficult and it has been solved analytically only for the zone boundary mode $k=N / 2$. In this case, one finds the critical energy $E_{\mathrm{c}}(\beta=1)$

$$
E_{\mathrm{c}}=\frac{2 N}{9} \sin ^{2}\left(\frac{\pi}{N}\right) \frac{7 \cos ^{2}(\pi / N)-1}{\left[3 \cos ^{2}(\pi / N)-1\right]^{2}} .
$$

Above this energy, the zone-boundary mode solution looses stability by developing a spatial modulation. The initial zig-zag spatial pattern deforms in such a way to create a smooth long-wavelength envelope, with many bumps. Since the critical energy vanishes as $N$ increases, in the thermodynamic limit the zone-boundary mode is always unstable. However, the rate at which the instability develops diverges with system size $N$ [70]. The development of the instability leads to the creation of a "chaotic breather," as discussed in Sects. 2.4.5 and 2.4.6

Such instabilities exist also for other invariant modes and set of invariant modes, but have not yet been studied carefully neither analytically nor numerically. For instance, it is well known that if the energy is initially put in even (odd) modes in a FPU chain with an even number of oscillators and periodic boundary condition, energy remains in the set of even (odd) modes forever, until a critical energy is reached above which energy is exchanged among the two sets. In fact the set of even (odd) modes is an invariant set, according to our definition.

Let us sketch the derivation of formula (2.74) following Dauxois et al. [138]. Due to periodic boundary conditions, the normal modes are plane waves of the form

$$
q_{n}(t)=\frac{a}{2}\left(\mathrm{e}^{\mathrm{i} \theta_{n}(t)}+\mathrm{e}^{-\mathrm{i} \theta_{n}(t)}\right)
$$

where $\theta_{n}(t)=q n-\omega t$ and $q=2 \pi k / N(k=-N / 2, \ldots, N / 2)$. The dispersion relation of nonlinear phonons in the RWA given by $(2.86)$ is $\omega^{2}(q)=4(1+$ $\Delta) \sin ^{2}(q / 2)$, where $\Delta=3 a^{2} \sin ^{2}(q / 2)$ takes into account the nonlinearity. Modulational instability is investigated by studying the linearized equation associated with the envelope of the carrier wave (2.75). Therefore, one introduces infinitesimal perturbations in the amplitude and phase and looks for solutions of the form

$$
\begin{aligned}
q_{n}(t) & =\frac{a}{2}\left[1+b_{n}(t)\right] \exp \left(\mathrm{i}\left[\theta_{n}(t)+\psi_{n}(t)\right]\right)+\frac{a}{2}\left[1+b_{n}(t)\right] \exp \left(\mathrm{i}\left[\theta_{n}(t)+\psi_{n}(t)\right]\right) \\
& =a\left[1+b_{n}(t)\right] \cos \left[q n-\omega t+\psi_{n}(t)\right],
\end{aligned}
$$

where $b_{n}$ and $\psi_{n}$ are reals and assumed to be small in comparison with the parameters of the carrier wave. Substituting (2.76) into the equations of motion, one obtains for the real and imaginary part of the secular term $\exp (\mathrm{i}(q n-\omega t))$ 


$$
\begin{aligned}
& -\omega^{2} b_{n}+2 \omega \dot{\psi}_{n}+\ddot{b}_{n}=(1+2 \Delta)\left[\cos q\left(b_{n+1}+b_{n-1}\right)-2 b_{n}\right] \\
& -\Delta\left(b_{n+1}+b_{n-1}-2 b_{n} \cos q\right)-(1+2 \Delta) \sin q\left(\psi_{n+1}-\psi_{n-1}\right) \\
& -\omega^{2} \psi_{n}-2 \omega \dot{b}_{n}+\ddot{\psi}_{n}=(1+2 \Delta)\left[\cos q\left(\psi_{n+1}+\psi_{n-1}\right)-2 \psi_{n}\right] \\
& +(1+2 \Delta) \sin q\left(b_{n+1}-b_{n-1}\right)+\Delta\left(\psi_{n+1}+\psi_{n-1}-2 \psi_{n} \cos q\right) .
\end{aligned}
$$

Further assuming $b_{n}=b_{0} \mathrm{e}^{\mathrm{i}(Q n-\Omega t)}+$ c.c. and $\psi_{n}=\psi_{0} \mathrm{e}^{\mathrm{i}(Q n-\Omega t)}+$ c.c. one gets the two following equations for the secular term $\mathrm{e}^{\mathrm{i}(Q n-\Omega t)}$

$$
\begin{aligned}
& b_{0}\left[\Omega^{2}+\omega^{2}+2(1+2 \Delta)(\cos q \cos Q-1)-2 \Delta(\cos Q-\cos q)\right] \\
& -2 i \psi_{0}[\omega \Omega+(1+2 \Delta) \sin q \sin Q]=0 \\
& \psi_{0}\left[\Omega^{2}+\omega^{2}+2(1+2 \Delta)(\cos q \cos Q-1)+2 \Delta(\cos Q-\cos q)\right] \\
& +2 i b_{0}[\omega \Omega+(1+2 \Delta) \sin q \sin Q]=0 .
\end{aligned}
$$

Nontrivial solutions for the linear system of (2.79)-(2.80) can be found only if the equations determinant vanishes, i.e. if the following equation is fulfilled:

$$
\begin{aligned}
& \left\{(\Omega+\omega)^{2}-4(1+2 \Delta) \sin ^{2}\left(\frac{q+Q}{2}\right)\right\} \times \\
& \times\left\{(\Omega-\omega)^{2}-4(1+2 \Delta) \sin ^{2}\left(\frac{q-Q}{2}\right)\right\}=4 \Delta^{2}(\cos Q-\cos q)^{2} .
\end{aligned}
$$

This equation admits four different solutions when the wavevectors $q$ of the unperturbed wave and $Q$ of the perturbation are fixed. If one of the solutions is complex, an instability of one of the modes $(q \pm Q)$ is present, with a growth rate equal to the imaginary part of the solution. Using this method, one can derive the instability threshold amplitude for any wavenumber. A first interesting case is $q=\pi$, the zone-boundary mode. One can easily see that (2.81) admits two real and two complex conjugate imaginary solutions if and only if

$$
\cos ^{2} \frac{Q}{2}>\frac{1+\Delta}{1+3 \Delta}
$$

The first mode to become unstable when increasing the amplitude $a$ corresponds to the wavenumber $Q=2 \pi / N$. Therefore, the critical amplitude $a_{\mathrm{c}}$ above which the $q=\pi$-mode looses stability is

$$
a_{\mathrm{c}}=\left(\frac{\sin ^{2}(\pi / N)}{3\left[3 \cos ^{2}(\pi / N)-1\right]}\right)^{1 / 2} .
$$

Since for the $\pi$-mode the energy is given by $E=N\left(2 a^{2}+4 a^{4}\right)$, one obtains the critical energy given in (2.74). The asymptotic behavior for large $N$ of this formula gives the same threshold as (2.99) in Sect. 2.4.6, see also [81, 139]. This critical energy is also very close to the Chirikov threshold for short wavelength (2.7). 


\subsubsection{Expansions for Generic Discrete Systems-Envelopes}

If we excite a high-frequency mode, $\gamma$, with $n \equiv N+1-\gamma \ll N+1$, then the instantaneous oscillator amplitude alternates from one oscillator to the next. As in previous studies [90, 91, 92], to remove this fast variations an envelope function $\psi_{i}(t)=(-1)^{i} q_{i}(t)$ is introduced, giving a smoothed spatial profile. The smoothed profile allows the oscillator to be described by a continuous variable from a Taylor expansion, giving

$$
\psi_{t t}+4 \psi+16 \beta \psi^{3}+\left\{\psi_{x x}+12 \beta\left(\psi \psi_{x}^{2}+\psi^{2} \psi_{x} x\right)\right\}+\cdots=0,
$$

where subscripts $t$ and $x$ stand for temporal and spatial derivatives of $\psi(x, t)$. Linear terms with spatial derivatives describe the dispersion, the dependence of the frequency $\omega$ on effective wave number $\pi k /(N+1)$ in (2.84), while nonlinear terms produce a frequency shift, that steepens the envelope function tending to form localized states (CBs). This process qualitatively explains why relaxation is accompanied by the formation of sharply localized states if energy is initially deposited in the high-frequency part of the spectrum, where the effect of dispersion is small, while only broad nonlinear structures are formed if the energy is initially in the low frequency modes where the dispersion is large [92]. Keeping the leading terms proportional to powers of degree zero and two and assuming a monochromatic dependence $\psi(x, t)=\psi(x) \cos (\omega t)$, leads to an equation for $\psi(x)$

$$
\left(-\omega^{2}+4\right) \psi+\psi_{x x}+\beta\left(12 \psi^{3}+9 \psi \psi_{x}^{2}+9 \psi^{2} \psi_{x x}\right)=0,
$$

where we have used the RWA, i.e. the expansion

$$
\cos ^{3}(\omega t)=(3 / 4) \cos (\omega t)+(1 / 4) \cos (3 \omega t),
$$

and dropped terms proportional to $\cos (3 \omega t)[90,91]$. Neglecting terms proportional to $\beta$ yields a linear equation for the eigenmodes:

$$
\left(-\omega^{2}+4\right) \psi+\psi_{x x}=0 .
$$

Solving (2.87) with zero boundary conditions at $x=0$ and at $x=N+1$ gives eigenmodes for $n=N+1-k \ll N+1$ which correspond to the high-frequency linear normal modes of the discrete FPU chain

$$
\psi_{n}^{(0)}(x)=\psi_{\max , n} \sin \left(q_{n} x\right), \quad \omega^{2}=4-q_{n}^{2}, \quad q_{n}=\frac{\pi n}{N+1} .
$$

The nonlinear equation (2.85) has exact analytical solutions, $\psi(x)$, which are periodic functions of $x$. There are three types of solutions:

(i) the infinite chain having a single localized breather with $\psi(x) \rightarrow 0$ as $x \rightarrow \pm \infty$, with frequency $\omega=\omega_{\mathrm{B}}$

$$
\omega_{\mathrm{B}}^{2}=4+6 \beta \psi_{m}^{2},
$$

where $\psi_{m}$ is the breather maximum amplitude given in (2.91); 
(ii) the chain with periodic boundary conditions including the $\pi$-mode for which each oscillator has opposite phase and equal amplitude as its neighbors, and, correspondingly, the envelope function $\psi=\psi_{\max }=\psi_{\min } \equiv \psi_{m}$, a constant, for which the nonlinear frequency shift reaches a maximum value

$$
\omega_{\mathrm{B}}^{2}=4+12 \beta \psi_{m}^{2},
$$

(iii) and the case of fixed zero displacement at $x=0$ and $x=N+1$, with intermediate values of $\omega_{\mathrm{B}}$.

For a single breather, $n=1$, the breather structure is similar to a breather on an infinite line which has analytic approximations for small and large amplitude. For $9 \beta \psi_{m}^{2} \ll 1$

$$
\psi_{\mathrm{B}}(x)=\frac{\psi_{m}}{\cosh \left(\sqrt{6 \beta} \psi_{m} x\right)},
$$

while in the large amplitude case $9 \beta \psi_{m}^{2} \gg 1$, the breather has a finite width of $4-5$ oscillators

$$
\psi_{\mathrm{B}}(x)=\psi_{m} \cos \sqrt{\frac{2}{3}} x \quad|x|<\pi \sqrt{\frac{3}{8}} .
$$

For most numerical studies of oscillator chains the initial state imposed on the system is that of a single linear mode. This state is generally not close to an equilibrium. The initial state rapidly relaxes, governed by the nonlinear equations. The evolution may be influenced by the underlying stability of nearby equilibria, but cannot be analyzed directly as perturbations around those equilibria. It is also possible to prepare the initial condition to be close to an equilibrium and consequently to directly analyze linear stability. The envelope solutions are fast oscillating functions of time which are subject to parametric (modulation) instability, i.e. an instability which is driven by the periodic variation of the frequency that appears in the linear equation for a perturbation. The frequency shift is caused by the nonlinearity in the unperturbed envelope solution. For the usually applied modal initial conditions, unstable breakup of modes is observed [87, 88]. However, numerical calculations show that the nonlinear stage of this instability leads to the formation of long-living self-organized localized structures, the chaotic breathers, which appear to be marginally stable with respect to a fast modulational instability.

Another question is how many breathers appear after the relatively short time of evolution from an initial state. In this context, fixed zero boundary conditions are significantly different from the $\pi$-mode initial values for periodic boundary conditions. In the periodic case, the $\pi$-mode is simultaneously a normal mode of the linear problem and an exact solution to the nonlinear envelope equation. Evolution from this equilibrium state is initiated by a modulation instability, and the wavelength of the fastest growing mode of the linearized equations gives an estimate of the number of breathers generated 
during the nonlinear phase of instability. In the case of zero boundary conditions, the high-frequency normal modes do not satisfy the nonlinear envelope equation. When used as initial conditions at low energy, they relax toward or around a few nearest stable equilibrium solutions. We expect that the linear analysis could, at best, only qualitatively describe their evolution.

\subsubsection{Instability from Short-Wavelength Initial Conditions}

For analysis of nonstationary envelopes, which describe relaxation, instability, or breather translational motion, it is convenient to rewrite the basic equation (2.84) in the form of two coupled equations for amplitude $q(x, t)$ and phase $\phi(x, t)$

$$
\psi(x, t)=q(x, t) \cos (\omega t+\phi(x, t)) .
$$

Substituting (2.93) in (2.84) and collecting terms proportional to $\sin (\omega t+$ $\phi(x, t))$ and $\cos (\omega t+\phi(x, t))$ leads to coupled equations

$$
\begin{gathered}
q \phi_{t t}+2 q_{t}\left(\omega+\phi_{t}\right)+2 q_{x} \phi_{x}+q \phi_{x x}+12 \beta q^{2} q_{x} \phi_{x}+3 \beta q^{3} \phi_{x x}=0, \\
q_{t t}-\left(\omega+\phi_{t}\right)^{2} q+4 q+q_{x x}-q \phi_{x}^{2}+12 \beta q^{3}+9 \beta q\left(q_{x}\right)_{x}-6 \beta q^{3} \phi_{x}^{2}=0 .
\end{gathered}
$$

The frequency $\omega$ is a constant given approximately by (2.89) and determined by the amplitude of the unperturbed solution. When the amplitude is slightly varied, $q(x, t)=\psi(x)+\delta q(x, t)$, the frequency of the fast nonlinear oscillation is also varied. As $\omega$ is taken to be contant this effect is represented by the time-varying phase, $\phi(x, t)=\delta \phi(x, t)$. Since (2.94) depends on derivatives of $\delta \phi(x, t)$, but not the phase itself, it can be linearized by considering the derivatives of $\delta \phi(x, t)$ as first order corrections. This yields two coupled linear equations

$$
\begin{gathered}
2 \omega \boldsymbol{\delta} q_{t}+\psi \boldsymbol{\delta} \phi_{t t}+2 \psi_{x}\left(1+6 \beta \psi^{2}\right) \boldsymbol{\delta} \phi_{x}+\psi\left(1+3 \beta \psi^{2}\right) \boldsymbol{\delta} \phi_{x x}=0, \\
\delta q_{t t}+\left(\left(1+9 \beta \psi^{2}\right) \boldsymbol{\delta} q_{x}\right)_{x}+\left(4-\omega^{2}+36 \beta \psi^{2}+18 \beta \psi \psi_{x x}+9 \beta \psi_{x}^{2}\right) \boldsymbol{\delta} q-2 \omega \psi \boldsymbol{\delta} \phi_{t}=0 .
\end{gathered}
$$

These equations have been solved numerically under various assumptions, with the result being that breather equilibria in chains with fixed ends are probably marginally stable to parametric instabilities [93]. This probably accounts for the long-time stability of the breathers that are formed from the parametric instabilities of mode initial conditions. To explore the latter situation a useful approximation is to consider the case of constant spatial profile of the envelope $\psi(x, t)=\psi_{m} \cos \omega t$. This corresponds to the $\pi$-mode with periodic boundary conditions, which has the highest nonlinear frequency shift (2.90) [91]. This mode is a solution to (2.85) but does not belong to the envelope solutions with zero boundary conditions. Setting the spatial derivative of $\psi(x)$ equal to zero, (2.96) and (2.97) reduce to coupled equations for $\delta \phi(x, t)$ and $\delta q(x, t)$, with constant coefficients. They can be solved by letting 
$\delta q(x, t) \propto \delta \phi(x, t) \propto \exp (s t+\mathrm{i} k x)$ which gives a biquadratic equation for $s$. Substituting for $\omega$ from (2.90), the result is

$$
\begin{aligned}
& s^{4}+2\left[36 y+8-k^{2}(1+6 y)\right] s^{2}=k^{2}(1+3 y)\left[24 y-k^{2}(1+9 y)\right], \\
& y=\beta \psi_{m}^{2} .
\end{aligned}
$$

This gives a threshold for the modulation instability of the $\pi$-mode

$$
6 \beta \psi_{m}^{2} \frac{(N+1)^{2}}{\pi^{2}}>1
$$

There is a most unstable wavenumber $k_{m}$ which corresponds to the maximum of the growth rate, $s_{m}$. In the limit of small $\psi_{m}, 9 y \ll 1$, the value of $k_{m}$ and $s_{m}$ is found by dropping $s^{4}$, then setting $\mathrm{d}\left(s^{2}\right) / \mathrm{d}\left(k^{2}\right)=0$, to obtain [91]

$$
k_{m}=\sqrt{12 \beta} \psi_{m}, \quad s_{m}=3 \beta \psi_{m}^{2} .
$$

For intermediate amplitude envelopes all terms are included in (2.98). In the limit of large amplitudes $9 y \gg 1$, the fastest growing mode has wavenumber and maximum growth rate [92]

$$
k_{m}=1.23, \quad s_{m}=0.93 \sqrt{\beta} \psi_{m} .
$$

Comparing (2.100) and (2.101), the transition from small to large amplitude takes place at $\beta \psi_{m}^{2} \simeq 1 / 9$ which corresponds, for $\beta=0.1$, to $\psi_{m} \simeq 1$.

These results have been checked by numerical calculations, starting from various mode initial conditions, obtaining reasonable agreement [93]. For example, in one way of forming initial conditions one can take a set of Fourier modes to approximate a square wave, using $a_{n}=4 / \pi n$, $n$ odd, with $n=N+1-\gamma(\gamma$ is an initial $k$-value $)$. Considering the nine highest frequency modes, for $E=16$, one obtains the evolution as shown, at three times, in Fig. 2.8. The initial nine ripples, with large end-values, in Fig. 2.8a, are characteristic of the Fourier sum. The evolution through various transitions, e.g., Fig. $2.8 \mathrm{~b}$ at $t=100$, leads to a large amplitude fastest growing mode at $t=220$ with a wavelength of $\lambda=16$, Fig. 2.8c, which is predicted from (2.100). The growth time from the first emergence of the fastest growing mode (not shown) is also consistent with the observations. The fastest growing $k$-value is established from either a smaller or a larger number of initial modes than the corresponding value $n_{m}=128\left(2 \pi / k_{m}\right)$. In either case, the subsequent time evolution, on a slower time-scale, is to form nonlinear chaotic breathers which coalesce and then decay to equipartition, on slower time-scales.

For the $\phi^{4}$ oscillator chain, starting from (2.9), one can make the same Taylor expansion used for the FPU chain, followed by the rotating wave approximation, to obtain [94]

$$
\left(\omega^{2}+m^{2}+4\right) \psi+\psi_{x x}+\frac{3}{4} \beta \psi^{3}=0,
$$



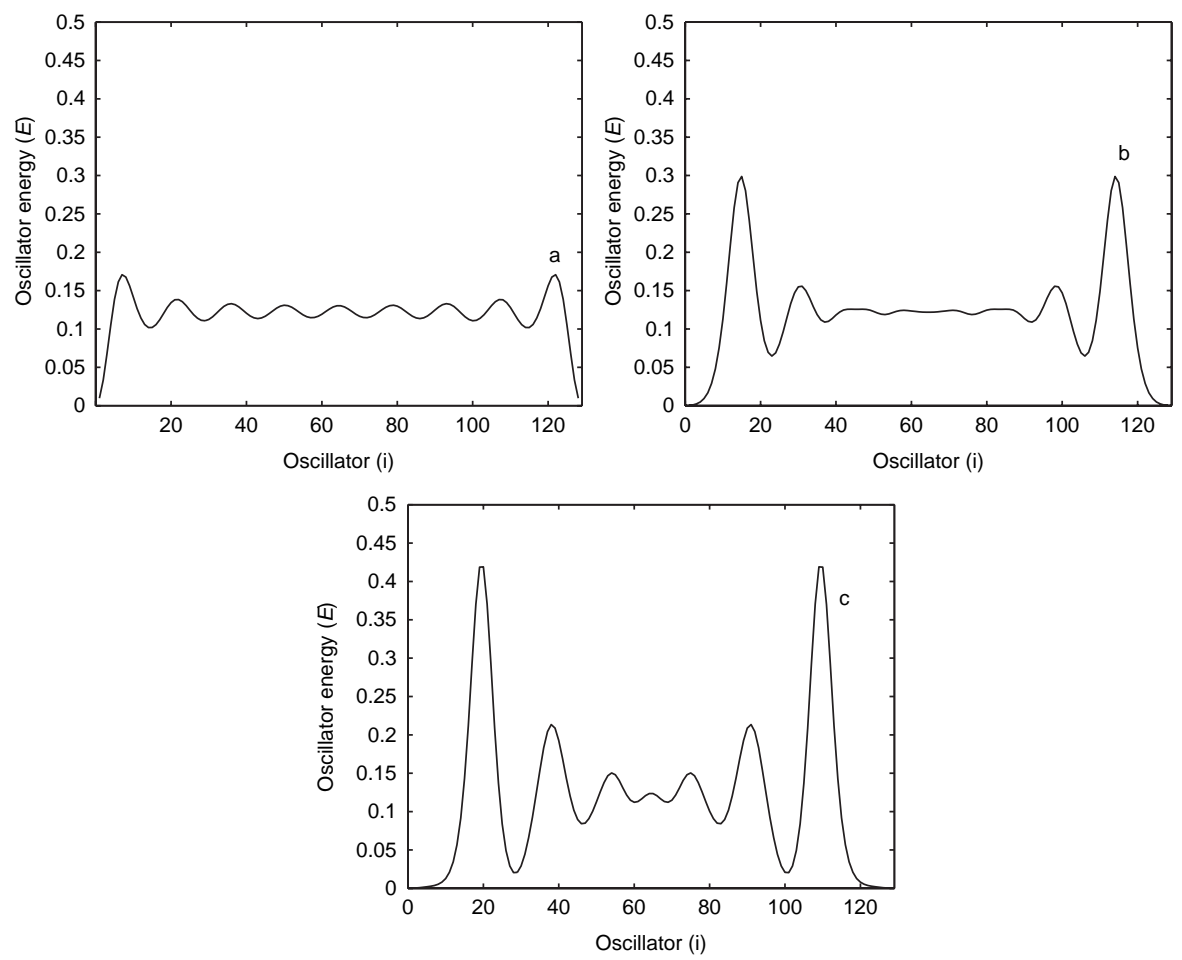

Fig. 2.8. Illustrating a set of initial conditions fixed at the ends but with a 9 -mode Fourier spectrum approximating a $\pi$-mode distribution in the central region, at times: (a) $t=10$, (b) $t=100$, (c) $t=220$. The predicted fastest growing mode has $\lambda_{m}=16$, in agreement with the results seen in (c).

which is to be compared with (2.85). One observes three differences: the extra linear coefficient $m^{2}$, the smaller coefficient of the $\psi^{3}$ term $(3 / 4$ rather than 12), and the absence of the mixed cubic terms. For most comparisons, we choose $m$ small ( $m=0.1$ ), which is negligible compared to 4 , and the mixed cubic term in the FPU plays only a minor role in the dynamics. The remaining clear difference is the factor of 16 that the $\psi^{3}$ term is smaller in the $\phi^{4}$ envelope than the FPU envelope. This factor is clearly understood by a comparison of the original FPU and $\phi^{4}$ Hamiltonians (2.1) and (2.9). Since the nonlinear $\phi^{4}$ potential results from a self force, the potential arises only from the extension of the mass point $q_{i}$ and is therefore quartic in that extension. In contrast, the FPU quartic potential arises from the difference of neighboring oscillators $\left(q_{i+1}-q_{i}\right)$. Since these oscillators are approximately $\pi$ out of phase, there is an increase of 4 factors of 2 in the quartic potential, i.e. 16. The effect of the factor of 16 is reflected in all subsequent calculations, appearing linearly in the instability threshold (2.99), as a square-root in the most unstable wavenumber $k_{m}(2.100)$ and linearly in the maximum growth rate $s_{m}(2.100)$, which were 
found numerically in comparisons between the two chains [94]. Care must be taken in the comparisons if the energy $E$ is used as the parameter, because there is a range of scalings of $\psi_{m}$ with $E$ from low to high energy (see $[93,94]$, for a detailed treatment). We will return to these comparisons of scalings in later sections describing the stochastic behavior.

In Sect. 2.4.5 we saw that the envelope approximation with periodic boundary conditions has an equilibrium solution that is exactly constant, the boundary or $\pi$-mode. This equilibrium becomes unstable at an envelope amplitude given by (2.99), and, using the low amplitude approximation for the energy,

$$
E \approx 2 N \psi_{m}^{2}
$$

then (2.99) yields the energy border of instability

$$
\beta E>\frac{\pi^{2}}{3 N} .
$$

This result can also be found directly from the discrete $\pi$-mode [70], so does not depend on the envelope expansion. For the FPU- $\beta$ chain with fixed ends, the instability still exists, but is only approximately given by (2.104), as the envelope equilibrium no longer has an expansion that yields an exact border of instability. Although the envelope equilibrium of the $\pi$-mode results from breather-like equations, it is an extended mode, rather than the usually studied intrinsically localized modes (ILMs), which are much more stable. This general class of short-wavelength modes, near the $\pi$-boundary mode, had previously been studied by Berman and Kolovskii [81] using a different technique. Starting from the mode representation for the FPU- $\beta$ chain, and assuming that only a few modes neighboring the $\pi$-mode were present, they expanded about that wave number $k_{\pi}$ as $n=k-k_{\pi}\left(n \ll k_{\pi}\right)$, and removing the fast oscillations by factoring out $\exp \left[\left(\mathrm{i} \omega_{\pi}+\lambda n\right) t\right], \lambda=2(\pi / N) \cos \left(\pi k_{\pi} / N\right)$, from the modes, they arrive at the nonlinear Schrödinger (NLS) equation

$$
i \frac{\partial \Phi}{\partial t}=\Omega \frac{\partial^{2} \Phi}{\partial \theta^{2}}+V_{0}|\Phi|^{2} \Phi
$$

where $V_{0}=(3 \beta / N) \sin ^{2}\left(\pi k_{\pi} / N\right), \Omega=(\pi / N)^{2} \sin \left(\pi k_{\pi} / N\right)$, and $\Phi(\theta, t)=$ $\Phi(\theta+2 \pi, t)$. The NLS equation, like the KdV equation, is completely integrable with soliton-like solutions. However, like the mKdV equation, the solitons are subject to an instability which can be calculated by linearization of the equilibrium, as outlined for the envelope approximation in Sect. 2.4.5. The result, for instability, is the same as in (2.104), but now involves a narrow mode packet, rather than the single $\pi$-mode. It is also close to the Chirikov mode overlap criterion for local chaos, which from (2.7) can be written as $\beta E \approx 2 \pi^{2} / 3 N$. As already discussed, all of these criteria signal the onset of local chaos in the discrete chain, but do not inform us about equipartition on nonexponential time-scales. However, the Berman-Kolovskii paper considers a second transition at which the narrow packet approximation breaks down and 
therefore the NLS approximation is no longer valid. For this higher energy, the packet size is not contained, which may lead to power-law time-scales to equipartition. The value they estimate is

$$
\beta E>\frac{2 \pi^{2}}{3},
$$

which is independent of $N$. This high-frequency estimate is quite close to the low-frequency estimate (2.50), both predicting nonexponentially slow diffusion, which survives in the thermodynamic limit. Both (2.106) and (2.50) give results for extended modes, which is the relevant result for low frequencies; but for high frequencies (2.106) does not take into account the later developments, predicting the formation of long-lived but not fully stable ILMs (the CBs). The original work included qualitative numerical support for both (2.104) and (2.106). A more detailed numerical investigation, including the formation and slow decay of the CBs, is given in Sect. 2.6. Some recent comments on, and reference to extensions of, the narrow packet approximation can be found in $[140]$.

\subsubsection{Geometric Formalism and the Method of Estimating the Largest Lyapunov Exponent}

As already mentioned in the Introduction, classical perturbation theory (CPT) is inadequate to describe the properties of Hamiltonian dynamics when the phase space is formed by chaotic orbits, even if a perturbative description is justified when the time-scales are short with respect to the instability time-scales. For the transition from weak stochasticity to strong stochasticity in high-dimensional systems, the perturbative treatment is completely inadequate, due to the energies involved which are much larger than the values for which it is meaningful to consider the systems as quasi-integrable. Moreover, the canonical transformation from natural coordinates to angle-action variables, which is a prerequisite to tackle chaos from the point of view of homoclinic intersections, is very complicated and necessarily approximated, not to speak of the lack of the generalization of the Poincaré-Birkhoff theorem at arbitrary $N$ concerning the fate of resonant tori, another necessary prerequisite for the standard description of chaos through homoclinic intersections.

A problem that naturally arises is how to explain the origin of a Strong Stochasticity Threshold (SST) and how to compute, the crossover energy. According to the above arguments, one has to look for some nonperturbative method. The only rigorous theoretical framework dealing with the opposite situation of CPT, i.e. with completely chaotic trajectories, is ergodic theory. We have already mentioned in Sect. 2.1.3 that it was Krylov who first realized the relevance of mixing for statistical mechanics, and the relevance of the stability properties of geodesics on Riemannian manifolds of negative curvature for mixing. More recently, the geometric approach has been reconsidered with the aid of numerical simulations, finding out that the dominant mechanism 
for dynamical instability in physically relevant geodesics flows is parametric instability due to curvature variations along the geodesics, instead of the negative curvature $[141,142,143,144]$.

For a dynamical system described by the Lagrangian function

$$
L(q, \dot{q})=\frac{1}{2} a_{i k}(q) \dot{q}^{i} \dot{q}^{k}-V(q)
$$

according to Maupertuis' principle of stationary action, among all the possible isoenergetic paths $\gamma(t)$ with fixed end points, the paths which make the first variation of the action functional vanish, which is such that

$$
\delta \mathcal{A}=\delta \int_{\gamma(t)} p_{i} \mathrm{~d} q_{i}=\delta \int_{\gamma(t)} \frac{\partial L}{\partial \dot{q}_{i}} \dot{q}_{i} \mathrm{~d} t=0
$$

are natural motions.

Since $2 W=\dot{q}_{i} \partial L / \partial \dot{q}_{i}$, is the kinetic energy, Maupertuis' principle reads

$$
\delta \int_{\gamma(t)} 2 W \mathrm{~d} t=\delta \int_{\gamma(t)}\left(g_{i k} \dot{q}^{i} \dot{q}^{k}\right)^{1 / 2} \mathrm{~d} t=\delta \int_{\gamma(s)} \mathrm{d} s=0 .
$$

The last integral indicates that if the configuration space $M$ of a system with $N$ degrees of freedom is given a proper Riemannian structure by introducing the metric $[42,145]$

$$
g_{i k}=2[E-V(q)] a_{i k}
$$

so that $\mathrm{d} s^{2}=4[E-V(q)] a_{i j} \mathrm{~d} q^{i} \mathrm{~d} q^{j}$ is its arclength, then the trajectories of the Newtonian motions coincide with the geodesics of the manifold $M$ endowed with the metric tensor (2.110). This metric is known as the Jacobi metric and is defined in the region of the configuration space where $E>V(q)$. In local coordinates, the geodesic equations on a Riemannian manifold are given by

$$
\frac{\mathrm{d}^{2} q^{i}}{\mathrm{~d} s^{2}}+\Gamma_{j k}^{i} \frac{\mathrm{d} q^{j}}{\mathrm{~d} s} \frac{\mathrm{d} q^{k}}{\mathrm{~d} s}=0
$$

where $s$ is the proper time and $\Gamma_{j k}^{i}$ are the Christoffel coefficients of the LeviCivita connection associated with $g_{i k}$ [146]. By direct computation, it can be easily verified that the geodesic equations, together with the relation between $s$ and $t$, i.e. $\mathrm{d} s^{2}=4[E-V(q)] \mathrm{d} t^{2}$, yield

$$
\frac{\mathrm{d}^{2} q^{i}}{\mathrm{~d} t^{2}}=-\frac{\partial V}{\partial q^{i}}
$$

i.e. Newton's equations associated with the Lagrangian (2.107).

The stability of an orbit is related to the curvature of the Riemannian manifold. If we define the curvature $K$ at a point $x$ relative to a tangent plane $\pi_{a}$ which is spanned by the vectors $u, v$ at $x$, then

$$
K(u, v)=K(x, \pi)=\frac{\langle R(v, u) u, v\rangle}{|u \wedge v|^{2}},
$$


where $R$ is the Riemann-Christoffel curvature tensor [146]. $K$ turns out to be independent of the choice of the two vectors $u, v$ in $\pi$. The knowledge of $K$ for the $N(N-1)$ planes $\pi$ spanned by a maximal set of linearly independent vectors completely determines $R$ at $x$. If $\operatorname{dim}(M)=2$ then $K$ coincides with the Gaussian curvature. A manifold is isotropic if $K(x, \pi)$ does not depend on the choice of the plane $\pi$. The remarkable result (Schur's theorem [146]) is that in this case $K$ is also constant, i.e. it also does not depend on the point $x$. For a constant-curvature, i.e. isotropic, manifold (2.113) reduces to a constant

$$
K=\frac{1}{N(N-1)} \mathcal{R},
$$

where $N$ is the number of degrees of freedom and $\mathcal{R}$ is the scalar curvature.

For a congruence of geodesics $\left\{\gamma_{\tau}(s)=\gamma(s, \tau) \mid \tau \in R\right\}$ issuing from a neighborhood $\mathcal{I}$ of a point of a manifold [for more details see [142]], dependent on the parameter $\tau$, fixing a reference geodesic $\bar{\gamma}\left(s, \tau_{0}\right)$, if $\dot{\gamma}(s)$ is the vector field tangent to $\bar{\gamma}$ in $s$, and $J(s)$ the vector field tangent in $\tau_{0}$ to the curves $\gamma_{s}(\tau)$ for a fixed $s$, then the evolution of $J$ contains the information on the stability (or instability) of the reference geodesic $\bar{\gamma}$; if $|J|$ grows exponentially, then the geodesic will be unstable in the Lyapunov sense, otherwise it will be stable. It is remarkable that such an evolution is completely determined by the curvature tensor $R$, which is a consequence of the fact that $J$ is a Jacobi field, i.e. it obeys the equation

$$
\nabla_{\dot{\gamma}}^{2} J(s)+R(J(s), \dot{\gamma}(s)) \dot{\gamma}(s)=0 \text {. }
$$

Among several Riemannian geometrizations of Newtonian dynamics, a very interesting one is defined in an enlarged configuration spacetime $M \times R^{2}$, with local coordinates $\left(q^{0}, q^{1}, \ldots, q^{i}, \ldots, q^{N}, q^{N+1}\right)$, endowed with a nondegenerate pseudo-Riemannian metric whose arc-length [147]

$$
\mathrm{d} s^{2}=g_{\mu \nu} \mathrm{d} q^{\mu} \mathrm{d} q^{\nu}=a_{i j} \mathrm{~d} q^{i} \mathrm{~d} q^{j}-2 V(q)\left(\mathrm{d} q^{0}\right)^{2}+2 \mathrm{~d} q^{0} \mathrm{~d} q^{N+1}
$$

is called the Eisenhart metric. The natural motions are obtained as the canonical projection on the configuration space-time of those geodesics for which the arclength is positive-definite and given by $\mathrm{d} s^{2}=(\text { const. })^{2} \mathrm{~d} t^{2}$. The geometric formulations of Newtonian dynamics, based on Jacobi and Eisenhart metrics, respectively, are equivalent. The interest in the Eisenhart metric is that the instability equation for the geodesic spread (2.115) written in this metric yields the standard tangent dynamics equation which is commonly used in numerical computations of Lyapunov exponents.

We note, parenthetically, that the two basic topological conditions for the onset of chaos in any deterministic dynamics are stretching and folding of volumes in phase space [148]. In the case of Hamiltonian chaos, these two conditions are fulfilled by the existence of homoclinic intersections [15, 148]. In the Riemannian description of Hamiltonian chaos, stretching of nearby trajectories is provided by instability, and folding by not allowing the distance 
to grow indefinitely, i.e. by compactness. In this way, the phase trajectories forget the initial conditions; their evolution becomes unpredictable in the long run. In the majority of systems of physical interest, the configuration space is a bounded domain so that the instability of nearby trajectories, studied by means of (2.115), implies chaos.

In the particular case of isotropic (or constant curvature) manifolds, (2.115) becomes very simple: choosing a geodesic frame, i.e. a reference frame transported along a reference geodesic, the Jacobi equation is written as

$$
\frac{\mathrm{d}^{2} J}{\mathrm{~d} s^{2}}+K J=0
$$

and has either bounded oscillating solutions $\|J\| \propto \cos (\sqrt{K} s)$ or exponentially unstable solutions $\|J\| \propto \exp (\sqrt{-K} s)$ according to the sign of the constant sectional curvature $K$. If the curvatures are negative, the geodesic flow is unstable even if the manifold is no longer isotropic. Equation (2.117) is valid only if $K$ is constant. Nevertheless, for $\operatorname{dim} M=2$ (surfaces), the Jacobi equation, again written in a geodesic reference frame for the sake of simplicity, takes a form very close to that of isotropic manifolds,

$$
\frac{\mathrm{d}^{2} J}{\mathrm{~d} s^{2}}+\frac{1}{2} \mathcal{R}(s) J=0,
$$

where $\mathcal{R}(s)$ denotes the scalar curvature of the manifold at the point $P=\gamma(s)$. This equation helps in understanding the origin of geodesic instability besides hyperbolicity. In fact, the solutions of (2.118) may exhibit an exponentially growing envelope even if the curvature $\mathcal{R}(s)$ is everywhere positive but non constant. This is the case, for example, of two harmonic oscillators coupled through cubic or quartic terms [143, 149].

In many physically relevant systems (typically a set of coupled anharmonic oscillators on a lattice in $d$ space-dimensions) the curvatures are neither constant nor everywhere negative, and the straightforward approach based on (2.117) does not apply. This is the main difficulty in extending the methods of abstract ergodic theory to physically relevant models. The key point is to realize that negative curvatures are not strictly necessary for chaos, with the bumpiness of the manifold, being responsible for curvature fluctuations along the geodesics, that can trigger parametric instability and hence exponentially growing solutions of the stability equation (2.118).

In the large $N$ case, under a set of suitable hypotheses [for details see [60]], it is possible to derive a scalar effective stability equation resembling (2.118), where the role of $R(s)$ is played by a random process, from which an analytic estimate of the largest Lyapunov exponent can be obtained. The theory leads to the stochastic equation

$$
\frac{\mathrm{d}^{2} \psi}{\mathrm{d} s^{2}}+\left\langle k_{R}\right\rangle_{\Sigma_{E}} \psi+\left\langle\delta^{2} k_{R}\right\rangle_{\Sigma_{E}}^{1 / 2} \eta(s) \psi=0
$$


where $\psi$ denotes any of the components of $J$ in $(2.115)$ because all of them are assumed to obey the same effective equation of motion. Here $\left\langle k_{R}\right\rangle_{\Sigma_{E}}$ is the microcanonical average of the $1 / N$ fraction of the Ricci curvature $K_{\mathrm{R}}$ of the mechanical manifold ${ }^{2}$, and $\left\langle\delta^{2} k_{R}\right\rangle_{\Sigma_{E}} \equiv \frac{1}{N-1}\left\langle\delta^{2} K_{\mathrm{R}}\right\rangle_{\Sigma_{E}}$ is the variance of the Ricci curvature of the mechanical manifold averaged on the constant energy manifold $\Sigma_{E}=H^{-1}(E)$; finally, $\eta(s)$ is a Gaussian $\delta$-correlated random process of zero mean and unit variance.

Equation (2.119) is a scalar equation which, independently of the knowledge of dynamics, provides a measure of the average degree of instability of the dynamics through the growth-rate of $\psi(s)$. The peculiar properties of a given Hamiltonian system enter (2.119) through the global geometric properties $\left\langle k_{R}\right\rangle_{\Sigma_{E}}$ and $\left\langle\delta^{2} k_{R}\right\rangle_{\Sigma_{E}}$ of the ambient Riemannian manifold. Moreover these averages are functions of the energy $E$ of the system (and of the energy density $\varepsilon=E / N$, which is the relevant quantity for $N \rightarrow \infty)$, so that from (2.119) one can obtain the energy dependence of the geometric instability exponent.

Equation (2.119) is of the form

$$
\frac{\mathrm{d}^{2} \psi}{\mathrm{d} s^{2}}+\Omega(s) \psi=0
$$

representing a stochastic oscillator where the squared frequency $\Omega(s)$ is the above described stochastic Gaussian process. The process $\Omega(t)$, with proper time $s$ replaced by physical time $t$, is assumed to be stationary and $\delta$-correlated, that is its time correlation function $\Gamma_{\Omega}\left(t_{1}, t_{2}\right)$ is such that $\Gamma_{\Omega}\left(t_{1}, t_{2}\right)=\Gamma_{\Omega}\left(\left|t_{2}-t_{1}\right|\right)$ and $\Gamma_{\Omega}(t)=\tau \sigma_{\Omega}^{2} \delta(t)$, where $\tau$ is a characteristic time-scale of the process. The evaluation of this time-scale is still a rather delicate point, where some arbitrariness enters the theory. In studying various models an estimate has been successfully introduced, which combines the evaluation of the time needed to join two successive conjugate points along a geodesic (conjugate points are those points where the Jacobi field vanishes) with another time-scale which can be inferred by means of dimensional arguments. In [60] arguments are given which lead to the following two time-scales

$$
\tau_{1}=\left\langle\frac{\mathrm{d} t}{\mathrm{~d} s}\right\rangle \frac{\pi}{2 \sqrt{\Omega_{0}+\sigma_{\Omega}}}
$$

and

$$
\tau_{2}=\left\langle\frac{\mathrm{d} t}{\mathrm{~d} s}\right\rangle \frac{l^{2} \Omega_{0}}{6} \frac{2 \pi}{\sqrt{\Omega_{0}}} \simeq \frac{\Omega_{0}^{1 / 2}}{\sigma_{\Omega}},
$$

respectively, where $l$ is defined as $l=1 / \sqrt{\sigma_{\Omega}}$, and $\Omega_{0}=\left\langle\delta^{2} k_{R}\right\rangle_{\Sigma_{E}}, \sigma_{\Omega}=$ $\left\langle k_{R}\right\rangle_{\Sigma_{E}}$, whence $\tau$ in $\Gamma_{\Omega}(t)=\tau \sigma_{\Omega}^{2} \delta(t)$ is obtained by combining $\tau_{1}$ with $\tau_{2}$ as follows

${ }^{2}$ The Ricci curvature is the sum of the $N-1$ curvatures $K$, given in (2.113), relative to the $N-1$ planes spanned by a given vector $v$ and $N-1$ other (unit) vectors orthogonal to $v$. In components, $K_{\mathrm{R}}=R_{i j k}^{j} v^{i} v^{k}$, where $R_{i j k}^{j}$ are the components of the Riemann curvature tensor. 


$$
\tau^{-1}=2\left(\tau_{1}^{-1}+\tau_{2}^{-1}\right) .
$$

Whenever $\Omega(s)$ in $(2.120)$ has a nonvanishing stochastic component, the solution $\psi(s)$ is exponentially growing on the average [150]. By considering the proper time parametrization as a function of the physical time $t$, the estimate of the largest Lyapunov exponent from the physical time growthrate of $\left[\psi^{2}(t)+\dot{\psi}^{2}(t)\right]$ is then given by

$$
\lambda=\lim _{t \rightarrow \infty} \frac{1}{2 t} \log \frac{\psi^{2}(t)+\dot{\psi}^{2}(t)}{\psi^{2}(0)+\dot{\psi}^{2}(0)} .
$$

The ratio $\left(\psi^{2}(t)+\dot{\psi}^{2}(t)\right) /\left(\psi^{2}(0)+\dot{\psi}^{2}(0)\right)$ is computed by means of a technique developed by Van Kampen [150] and summarized in [60]. The main point is to compute the time evolution of the second moments of $\psi$ and $\dot{\psi}$, averaged over the realizations of the stochastic process. In general, the solutions of a stochastic oscillator equation as the one we are dealing with, are unstable. The envelope of an unstable solution exponentially grows in time with a growth rate $\lambda$ which — with the aid of Van Kampen's method - is found to be

$$
\begin{aligned}
\lambda\left(\Omega_{0}, \sigma_{\Omega}, \tau\right) & =\frac{1}{2}\left(\Lambda-\frac{4 \Omega_{0}}{3 \Lambda}\right) \\
\Lambda & =\left(2 \sigma_{\Omega}^{2} \tau+\sqrt{\left(\frac{4 \Omega_{0}}{3}\right)^{3}+\left(2 \sigma_{\Omega}^{2} \tau\right)^{2}}\right)^{1 / 3}
\end{aligned}
$$

The quantities $\Omega_{0}=\left\langle k_{R}\right\rangle_{\Sigma_{E}}, \sigma_{\Omega}=\left\langle\delta^{2} k_{R}\right\rangle_{\Sigma_{E}}$ and $\tau$ can be computed as static, i.e. microcanonical averages. Therefore (2.125) gives an analytic, though approximate, formula for the largest Lyapunov exponent independent of the numerical integration of the dynamics and of the tangent dynamics.

An important remark is that this analytic formula for $\lambda$ is derived under the geometric assumption of quasi-isotropy of the mechanical manifolds (for details see [60]), but this assumption is inadequate to tackle those systems whose mechanical manifolds are topologically nontrivial, in this case the theory has to take into account the role of an additional instability mechanism originating in the neighborhoods of the critical points of the potential function.

\subsection{Numerical Results: Relaxation to Equilibrium from Low-Frequency Modes}

\subsubsection{Observations of Diffusion: Numerical Determination of $\lambda$}

We have already discussed the early observations of periodicities among a few low-frequency modes, at low energies, and their explanation in terms of beat oscillations. We have also considered that solitons could be constructed from 
low-frequency mode initial conditions, which also produce the observed beats, i.e. another way of looking at the same phenomenon. Instability of single solitons in the mKdV equations was found to occur, for a given FPU- $\beta$ chain, at about the same energy at which the interaction of the beat oscillations generates stochastic layers (the concept of resonance overlap), and at the instability of an exact periodic solution. For large systems, at not too low energy, generic initial conditions would be expected to lie in the stochastic portion of the phase space and be able to diffuse to all portions of it by the Arnold diffusion mechanism. A stochasticity threshold (ST), to observable positive Lyapunov exponent, $\lambda$, and (possibly) an observable time to equipartition, $T_{\text {eq }}$, would be expected. In Sect. 2.3, we have discussed the numerical determination of

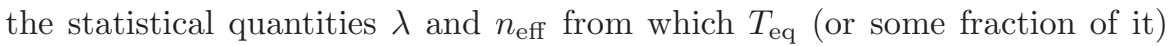
is found. In the higher energy regime, these and other statistical measures of the diffusion have been explored in the late 1970s and 1980s, particularly from the group in Firenze (see [50, 51, 52, 123, 128, 151]) and in Milano (see $[25,48,49,152,153])$. Here, and in Sect. 2.5.2, we present some of the numerical results, which are then used to guide further analytic studies. We restrict our numerical observations, in these sections, mainly to initial conditions of energy in low-frequency modes. The numerics starting from high-frequency modes have a somewhat different character, and will be treated separately in Sect. 2.6. Before presenting the results using the statistical measure, we first present in Fig. 2.9, a series of mode spectra, for $R=8, N=32$, above the $E_{\mathrm{c}}$
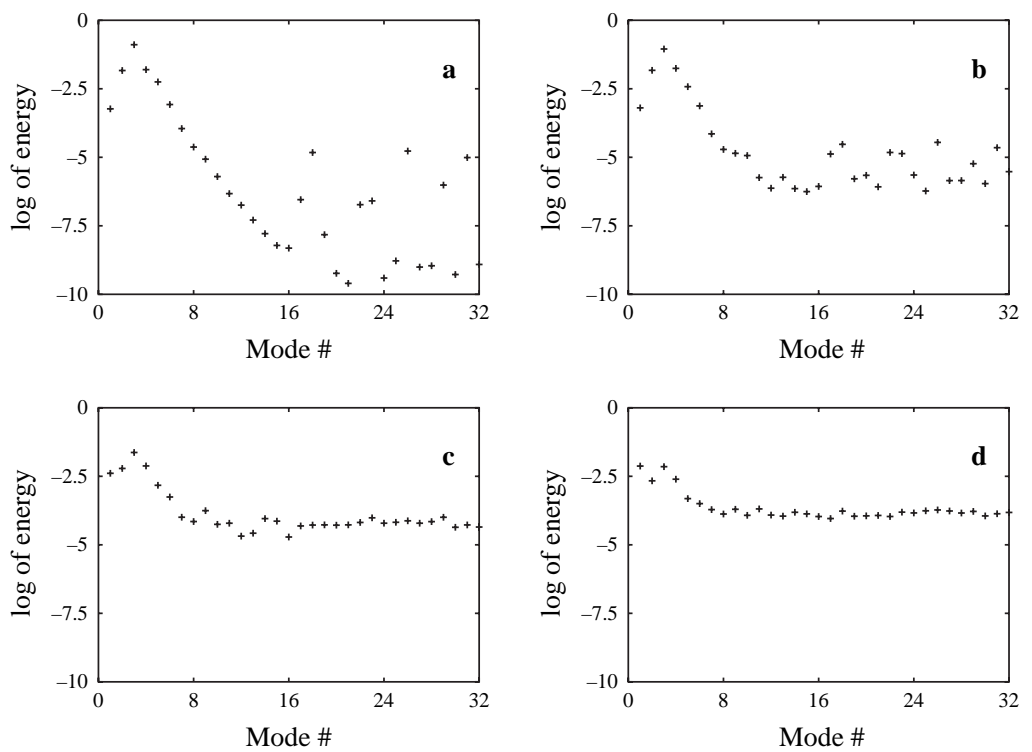

Fig. 2.9. $\log$ of average energies at $R=8.0, N=32(E=4)$ and four consecutive times $\tau=\gamma(\pi / N)^{2} t / 3, \gamma=3 ; \mathbf{a} \tau=2000, n_{\mathrm{eff}}=6.24 ; \mathbf{b} \tau=10,000, n_{\mathrm{eff}}=8.67$; c $\tau=40,000, n_{\text {eff }}=18.99$ and $\mathbf{d} \tau=78,000, n_{\text {eff }}=25.48$ 
transition as found in Fig. 2.4. The increase in energy in some high-frequency modes, specified from the selection rules, is well above the background, and continues to increase with time, finally resulting in equipartition. Note that the first frame in Fig. 2.9, with $\tau=2000$, corresponds to $N=32$ at $R=8$ in Fig. 2.4. The time has been normalized to scale away the number of oscillators in the chain. The values of $n_{\text {eff }}$, at each time, are listed in the figure caption.

The early statistical results, for the FPU- $\beta$ chain with $\beta$ chosen to be 0.1 , can be summed up with a graph from Pettini and Landolfi [50]. Choosing a range of $N$ from 64 to 512, and the initial cluster of low-frequency modes $\Delta k$, with $\Delta k / N$ fixed at $1 / 16$, they numerically calculated $\lambda(t)$ and $\eta(t)$ (see Sect. 2.3.2). To calculate for times that were not as long as $T_{\text {eq }}$ they fitted $\eta(t)$ to the stretched exponential

$$
\eta(t)= \begin{cases}\exp \left(-(t / \tau)^{\nu}\right), & t<\tau_{\mathrm{R}}, \\ \exp \left(-\left(\tau_{\mathrm{R}} / \tau\right)^{\nu}\right), & t>\tau_{\mathrm{R}}\end{cases}
$$

with $\tau_{\mathrm{R}}$ taken as a measure of $T_{\text {eq }}$. Their results for an asymptotic $\lambda$ and for $\tau_{\mathrm{R}}$ are shown together in Fig. 2.10, for a particular $N$ and $\Delta k$, but confirmed for other values of $N$ and $\Delta k$ with $\Delta k / N=$ const.. As shown in the figure, the slopes are power laws in $\varepsilon=E / N$, with a transition in the slope of $\lambda$ from $\lambda \propto \varepsilon^{2}$ at low $\varepsilon$ to $\lambda \propto \varepsilon^{2 / 3}$ at higher $\varepsilon$. They interpreted this transition as that from weak to strong stochasticity (the SST). The weaker scaling at higher $\varepsilon$ was predicted from an assumption of a fully random process, which might be expected to follow from strong mode overlap (see Sect. 2.8.1). We note from (2.7) that the transition roughly coincides with the prediction of mode overlap. Although not discussed in the original work, we also see the scaling $\tau_{\mathrm{R}} \propto \varepsilon^{-3}$, a scaling later confirmed for $T_{\text {eq }}$ from extensive calculations

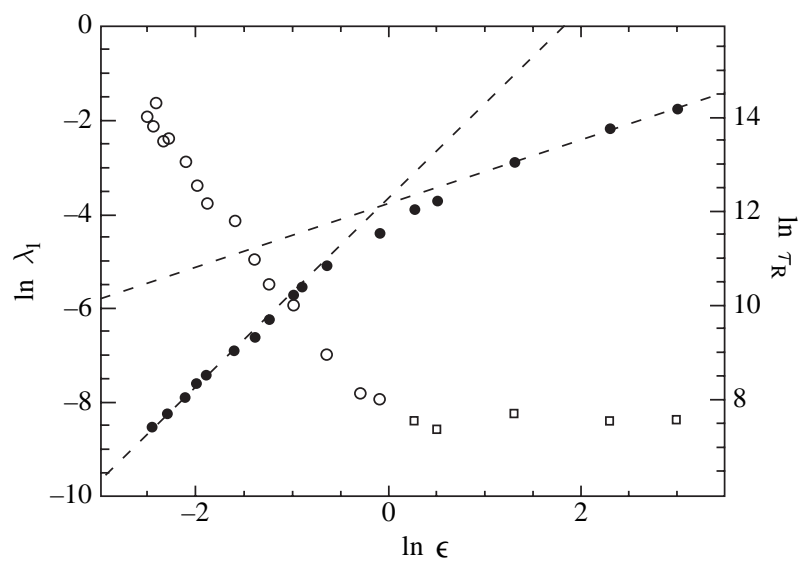

Fig. 2.10. The relaxation time $\tau_{\mathrm{R}}$ and the maximum Lyapunov exponent $\lambda$ vs. the energy per mode $\varepsilon=E / N$, for $N=128$ and $\Delta k=4$. Open circles and squares are relaxation times, and closed circles are the Lyapunov exponents 
as presented in the following subsection. The $\lambda$-scaling has been placed on a firm theoretical basis, as described in Sect. 2.4.7, with the theory compared to numerics in Sect. 2.8.2. Pettini and coworkers [61] have made extensive calculations of $\lambda(\varepsilon)$, using various initial conditions, for FPU- $\alpha$, FPU- $\beta$, and for the combination of the two, as given in (2.1). The FPU- $\alpha$ chain was particularly useful to explore a transition from power-law dependence of $\lambda$ on $\varepsilon$ to a condition in which $\lambda$ was not obtainable on computer accessible time-scales, which they called the stochasticity transition (ST). Their interpretation was that the ST gave a transition from essentially regular motion to chaotic motion. However, numerical determinations of $T_{\text {eq }}$ for the FPU- $\beta$ chain, as reviewed in the next section, interprets the more rapid increase of $T_{\text {eq }}$ with decreasing $\varepsilon$ as a transition from power-law $T_{\text {eq }} \propto \varepsilon^{-q}$ to exponential $T_{\text {eq }} \propto \exp \left(-a \varepsilon^{b}\right)$ variation of $T_{\text {eq }}$ with $\varepsilon$, as predicted by the Arnold diffusion mechanism (see $[55,57,58,65,121,134]$ ). We return to this unresolved question of whether or not there is a transition at small $\varepsilon$ to regular motion (no diffusion) after presenting the numerics here and in the next subsection.

The type of initial conditions typically used for the FPU- $\alpha$ system are one or a few modes, for which both short-time and long-time dynamics are observed. In Fig. 2.11 from Casetti et al. [61], we present values of $\lambda(\varepsilon)$ on $\log -\log$ scales for three values of $N=32$ (squares), 64 (triangles) and 128 (circles), starting from energy initially in the longest wavelength mode. The dominant scaling of $\lambda \propto \varepsilon^{5 / 3}$ is significantly weaker than the FPU- $\beta$ of $\lambda \propto \varepsilon^{2}$. At low values of $\varepsilon$ there is a clear drop-off to smaller values of $\lambda$ with a

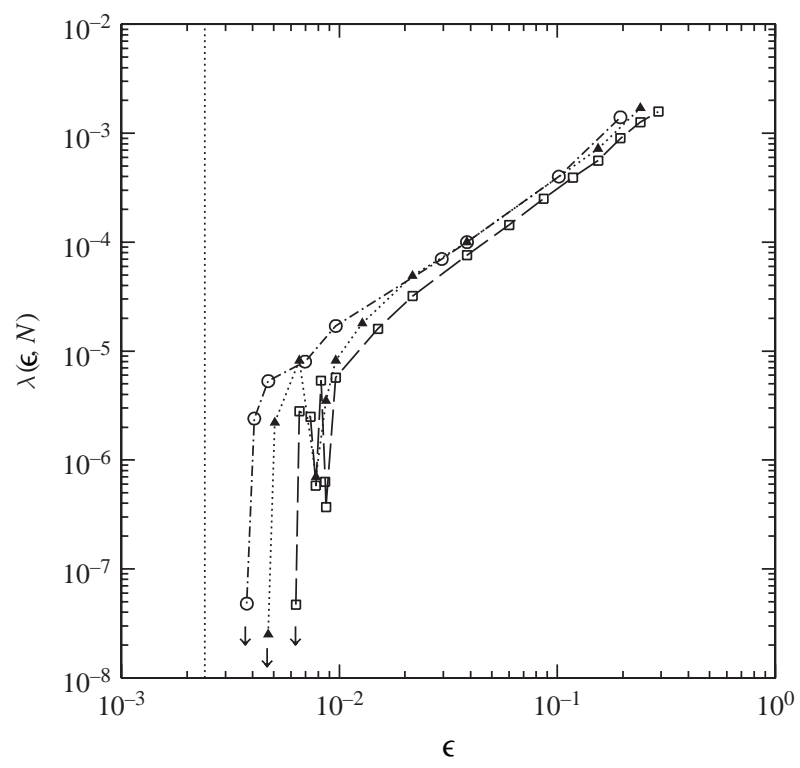

Fig. 2.11. Lyapunov vs. $\varepsilon$. Long-wavelength initial condition 
weak $N$-dependence, such that the drop-off occurs at larger $\varepsilon$ for smaller $N$. The results are slightly confused by an apparent resonant dip in $\lambda$ occurring near $\varepsilon=10^{-2}$. Another way of choosing initial conditions for measuring $\lambda$ is random over oscillators, which is also random over modes. This places the initial coordinates near equipartition to begin with, and therefore, should approximate the long-time average more rapidly. The results, shown in Fig. 2.12, include two small $N$-values, $N=8$ and 16, which cannot be compared directly with the previous figure, and may also include some "small- $N$ " effects. For the cases of $N=32$ (stars), and 64 (square stars), in the power-law regime, the same scaling $\lambda \propto \varepsilon^{5 / 3}$ is found, agreeing with the previous figure. Again, just considering the highest two $N$-values, the drop-off to significantly smaller $\lambda$ s have a scaling of $\varepsilon_{\mathrm{c}} N^{2} \approx$ const. (or $E_{\mathrm{c}} N \approx$ const.), where $\varepsilon_{\mathrm{c}}$ is the approximate break from the main scaling. This separation by $N$-value is much larger than in the previous figure. We note parenthetically that the scaling of this transition is the same as found for overlap of resonances in the FPU- $\beta$ system described in Sect. 2.4.1. We discuss these results further after obtaining numerical values of $T_{\text {eq }}$ in the following Subsection.

\subsubsection{Numerical Determination of $T_{\text {eq }}$ Scaling with $\varepsilon$}

Considerable numerical effort has gone into the determination of the scaling of $T_{\text {eq }}$ for the FPU- $\beta$ system and comparisons with systems with other force laws. For the FPU- $\beta$, Deluca et al. $[55,57,58]$, showed for a fixed $\varepsilon=E / N=0.5$,

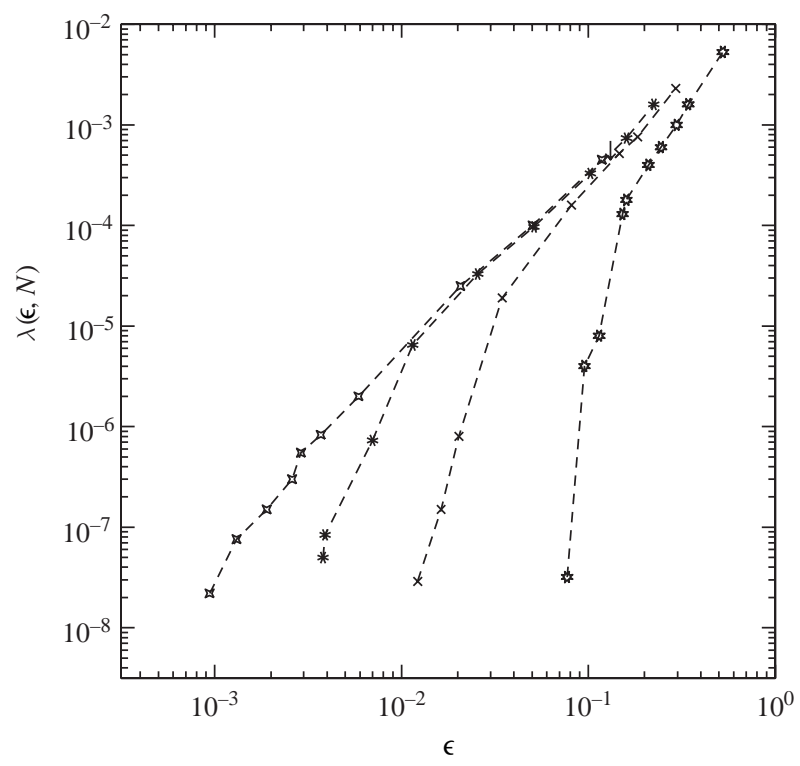

Fig. 2.12. Lyapunov vs. $\varepsilon$. Random initial condition 


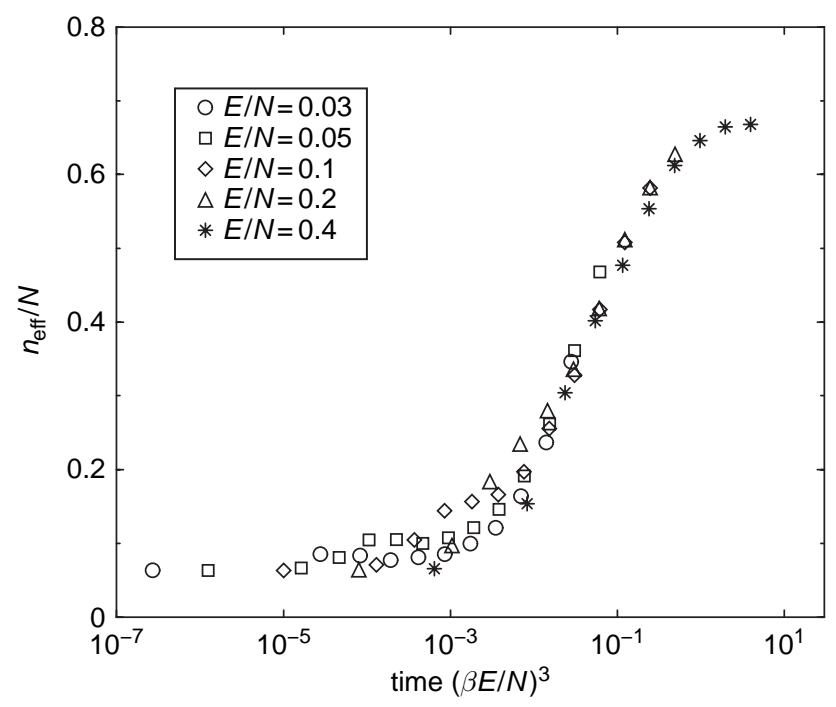

Fig. 2.13. Universal dependence of $n_{\mathrm{eff}} / N$ on $\varepsilon^{-3}$

over a range of relatively large $N$-values, $N=256-4096$, with a fixed percentage of initial modes, $\delta k / N=1 / 16$, that $n_{\mathrm{eff}}(t)$ fell on a universal curve, i.e. was independent of $N$, and therefore of $E$. For these relatively large $N$ values a previous weak dependence on $N$ with $\delta k$ held constant, was partly resolved by showing that $n_{\text {eff }}(t)$ has an initial transient, which mostly disappears for larger values of $n_{\text {eff }}$. This is a reasonable consequence of phase space ergodic mixing. With smaller values of $N$ investigated in other studies, there are significant weak $N$-dependences in the time to obtain equipartition. The universal dependence of $n_{\mathrm{eff}}$ on $\varepsilon^{-3}$ is illustrated in Fig. 2.13, where the time is normalized to $\tau=(\beta \varepsilon)^{3} t$. Since the times to equipartition become very long as $\varepsilon$ becomes small, only the largest values of $\varepsilon$ were integrated to $T_{\text {eq }}$ at $n_{\text {eff }}=0.65$. Because of the universal nature of the result shown in Fig. 2.13, the scaling of $T_{\text {eq }}$ with $\varepsilon$ should also be obtainable from a smaller value of $n_{\mathrm{eff}}$, say $n_{\mathrm{eff}}=0.4$. This was done, finding $t\left(n_{\mathrm{eff}}=0.4\right) \propto \varepsilon^{-3}$, but with some suggestion of steeper scaling at the smallest $\varepsilon$, interpreted in subsequent longer integrations $n_{\text {eff }}=0.65$ as a breakdown of power-law scaling if the initial driving frequency becomes too small [65]. A heuristic calculation of the observed scaling was given in [58], which we will summarize in Sect. 2.8.3. The results of DeLuca et al. [58] were reexamined by Berchialla et al. [59] for $N=511$, again for $t\left(n_{\text {eff }}=0.4\right)$, obtaining $\varepsilon^{-3}$ scaling with $\varepsilon$ in the same $\varepsilon$ range as previously, but extended to smaller $\varepsilon$-values where steeper scaling was observed. In addition to the $\log -\log$ plot, giving the $t \propto \varepsilon^{-3}$ scaling over part of the $\varepsilon$ range, a best-fit stretched-exponential dependence, $t \propto \exp \left(\varepsilon^{-1 / 4}\right)$, was also plotted showing a good fit to the smaller values of $\varepsilon$, as shown in Fig. 2.14, with the vertical dashed lines indicating the range of the data from 


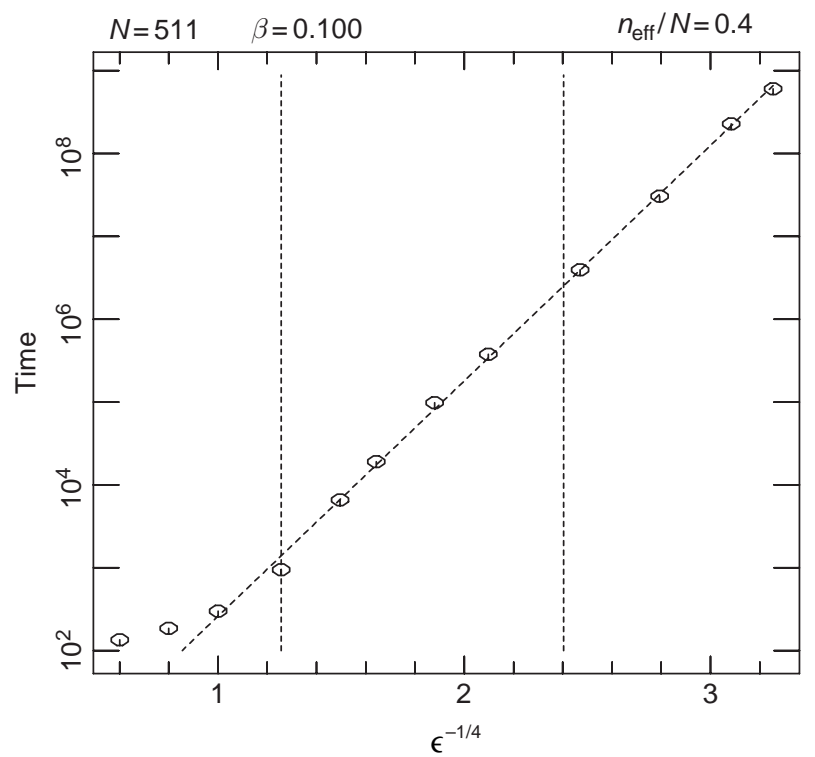

Fig. 2.14. Stretched exponential fit to the relaxation time

the DeLuca et al. [58] paper. (The values for $\varepsilon>1$ are considered to be above the SST and not of interest in the comparison). These results will be discussed further, after presenting additional data from other oscillator chains. An early comparison of the FPU- $\beta$ chain with the $\phi^{4}$ chain was made by Pettini and Cerruti-Sola [64]. Both oscillator chains have quartic nonlinear potentials, but the $\phi^{4}$ quartic term is on-site, while the FPU- $\beta$ quartic term is between oscillators. As already discussed in Sect. 2.2, the FPU- $\beta$ chain is simpler and easier to analyze. The early numerical comparison of the chains covered long-wavelength (low-frequency), short-wavelength (high-frequency) and intermediate-wavelength initial conditions. The recent theory, elucidating the more complex behavior, starting from high frequencies, had not been developed, but some general observations could be made. For long-wavelength initial conditions, at a given $\varepsilon$, the time to equipartition was shorter for the $\phi^{4}$ system than for the FPU- $\beta$ system. The opposite held at short wavelengths with $T_{\text {eq }}$ shorter for the FPU- $\beta$ than the $\phi^{4}$ system. It generally took longer to reach equipartition from short-wavelengths at a given $\varepsilon$. We have already discussed reasons for the different behavior and some short-time results in Sect. 2.4.1. Numerical results for short wavelengths will be given in Sect. 2.5. Here we present the detailed long-wavelength comparison as given in [65]. In Fig. 2.15 we compare the scaling of $T_{\text {eq }}(\varepsilon)$ for the FPU- $\beta$ and $\phi^{4}$ chains, each for two values of $N$. The nonlinear coefficient is $\beta=0.1$ for both chains, and $m=0.1$ for the $\phi^{4}$ chain. Except for the FPU- $\beta$ chain with $N=500$ (or 512), the other cases used $\Delta k / N=1 / 16$, as with the numerical results presented 


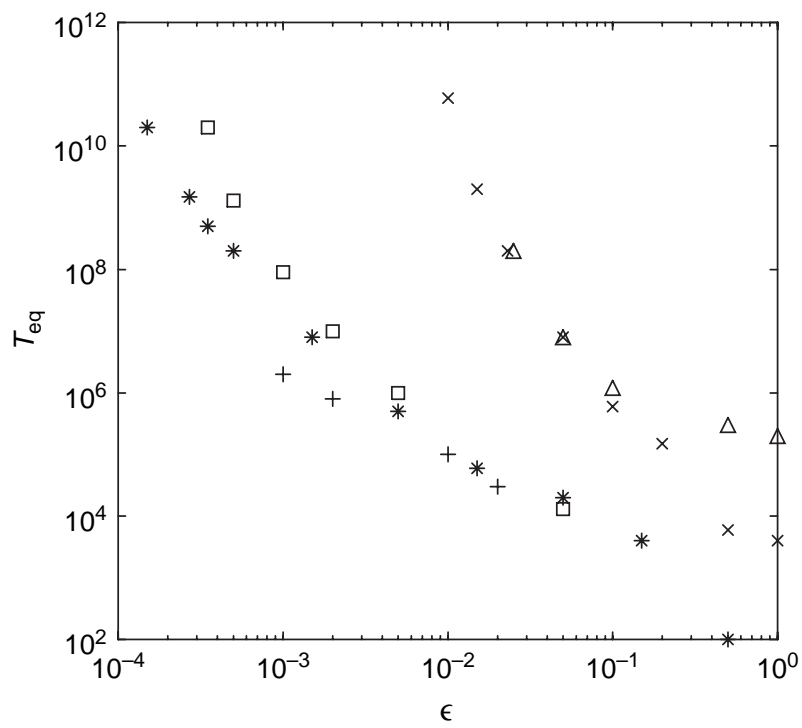

Fig. 2.15. Comparison of equipartition times for the FPU- $\beta[N=128$ (crosses), $N=512$ (triangles) $)$ and the $\phi^{4}$ model $(m=0.1, N=64$ (squares), $N=128$ (stars)]. The $\phi^{4}$ data with plusses are taken from [64]

in previous figures. For the $N=500,512$ data, some $\Delta k / N=1 / 16$ initial conditions and some $\Delta k=8(\Delta k / N=1 / 64)$ initial conditions were used. In all cases, the initial conditions used the lowest modes, equally excited. [For the two pairs of FPU- $\beta$ results, with large $N$, at $\varepsilon=0.5$ and $\varepsilon=1$, the lower points used $\Delta k / N=1 / 16(N=512)$ while the upper points used $\Delta k=8$ $(N=500)$.] However, although there is some initial $\Delta k / N$ dependence, the main reason for the separation was due to a statistical (and perhaps physical) anomaly that sometimes occurs at higher $N$-values. The criterion for determining $T_{\text {eq }}$ was the first time $n_{\text {eff }}(t)=0.65$. As discussed in Sect. 2.3.2 there are fluctuations on short time-scales which lead to $n_{\text {eff }}=0.65$ at equipartition, but these fluctuations also appear on the $n_{\text {eff }}(t)$ curves. Usually, $n_{\text {eff }}(t)$ crosses 0.65 and then fluctuates around that value. In the two upper values of $T_{\text {eq }}$, discussed above, the first fluctuation occurred just below $n_{\text {eff }}=0.65$, such that the first actual crossing was significantly later in time. Returning to discussion of the main results, the central portions of all curves display power-law scaling, with FPU- $\beta$ scaling giving $T_{\text {eq }}(\mathrm{FPU}-\beta) \propto \varepsilon^{-3}$. This is also seen when $t\left(n_{\mathrm{eff}}=0.4\right)$ is used to determine the scaling. The somewhat flatter scaling at large $\varepsilon$ is again interpreted as crossing the strong stochasticity threshold (SST). For the $\phi^{4}$ chain the central portion of the $\varepsilon$-values gives $T_{\text {eq }}\left(\phi^{4}\right) \propto \varepsilon^{-2.5}$, slightly flatter than the FPU- $\beta$ scaling. We will discuss this difference, together with a heuristic calculation of the scaling in Sect. 2.8.3. The $T_{\text {eq }}(\varepsilon)$ slopes become steeper at the lowest values of $\varepsilon$. For the $\phi^{4}$ chain, 
comparing the values of $T_{\text {eq }}$ for $N=64$ (squares) with the values of $N=128$ (stars), we see that for intermediate $\varepsilon$-values the two curves lie close together, agreeing with the expectations that $T_{\text {eq }}$ is a function of $\varepsilon$, only, [58, 121], as found in Sect. 2.8.3. However, at lower values of $\varepsilon$ we see that the $N=64$ points break away from the $N=128$ values, producing larger values of $T_{\text {eq }}$ with decreasing $\varepsilon$. This behavior was also found for the FPU system, and understood in terms of a critical value of energy, $E_{\mathrm{c}}$ for which the transition occurs. If the value of $E_{\mathrm{c}}$ is the same for all $N$, as calculated in Sect. 2.4.1 for the FPU system, then the value of $\varepsilon_{\mathrm{c}}$ at which the transition takes place would vary inversely with $N$ and therefore occur at a factor of 2 higher $\varepsilon$ for $N=64$ than for $N=128$, as observed in the figure. The values of $T_{\text {eq }}$ for the FPU chain for $N=128$ (crosses) and $N=512$ and 500 (triangles), would have the equivalent break at higher values of $T_{\text {eq }}$, which were not numerically reached. In Fig. 2.16, we plot $T_{\text {eq }}$, on a $\log$ scale, vs. $1 / \varepsilon$, for $N=128$ (stars) and for $N=64$ (squares). In the range in which the diffusion is exponentially slow (Arnold diffusion), then from the exponential scaling with $\delta \omega_{h} / \Omega_{\mathrm{B}} \propto 1 / E$, if $(2.54)$ is not satisfied, we expect to obtain a straight line for $\log \left(T_{\text {eq }}\right)$ vs. $1 / \varepsilon$ if $N$ is held constant. This is, indeed, found for 4 of the values, with a transition between $\varepsilon=10^{-3}$ and $5 \times 10^{-4}$, for $N=128$, and between $2 \times 10^{-3}$ and $10^{-3}$ for $N=64$, indicating that the change in dependence occurs at a fixed value of $E=E_{\mathrm{c}}$.

Because of the additional on-site parameter $m$, the numerical investigation of the $\phi^{4}$ chain involves a larger parameter space than the FPU chain. We can see from the linear mode frequencies, given in Sect. 2.2.2, that for $m \gg 1 / N$,

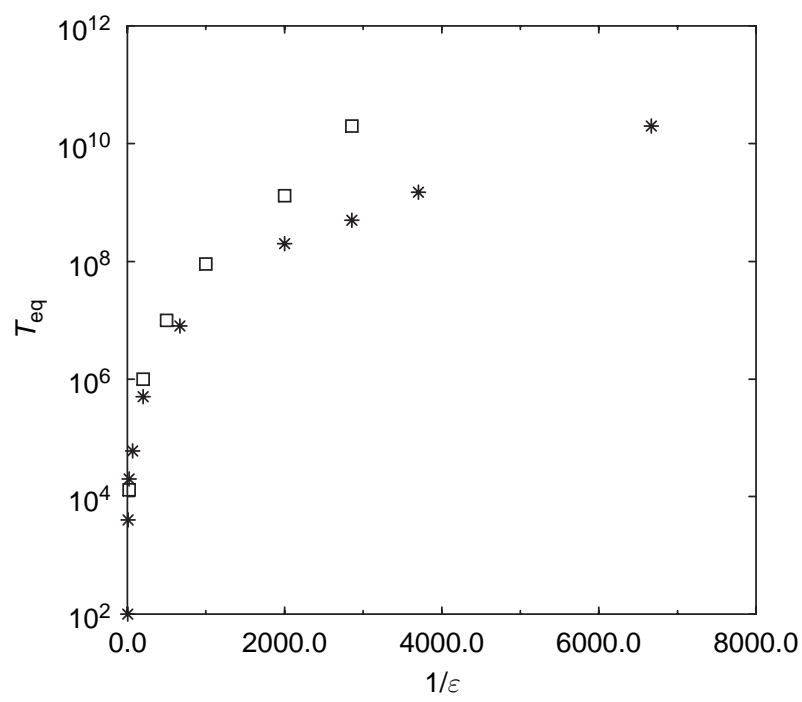

Fig. 2.16. Equipartition time for the FPU- $\beta$ model in the exponentially slow diffusion regime 


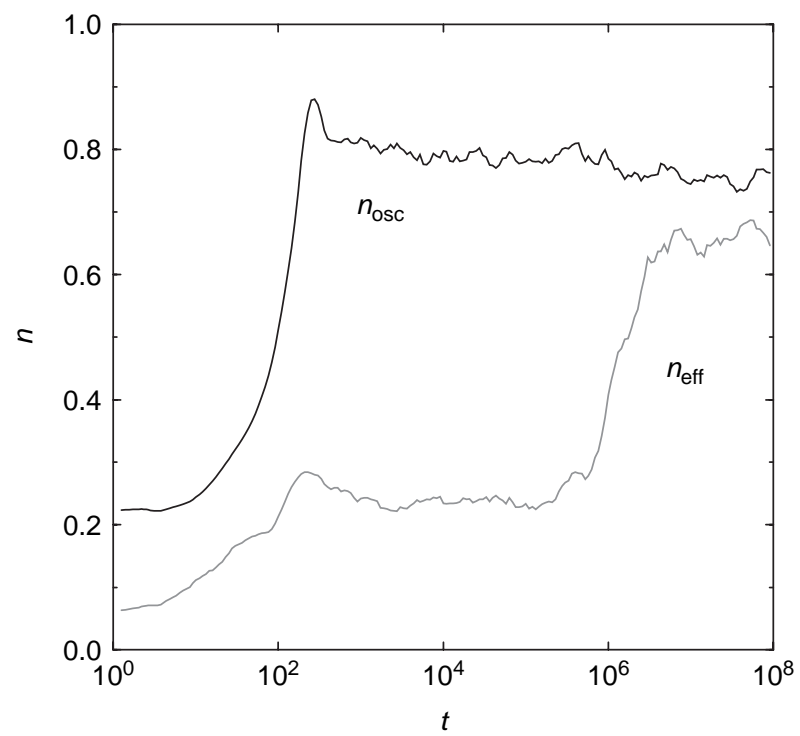

Fig. 2.17. Trapping in an intermediate state before the relaxation to equipartition begins, as illustrated by the time evolution of $n_{\text {eff }}$ and $n_{\text {osc }}$

the mode frequencies bunch together for long-wavelength modes, similar to high-frequency bunching. The result, starting from long-wavelength initial conditions, similar to what we shall see for both FPU- $\beta$ and $\phi^{4}$ at short wavelengths, is that there is trapping in a group of closely coupled modes that leads to a plateau in $n_{\text {eff }}$, before the continuation toward equipartion. We illustrate the plateau in Fig. 2.17, calculating both $n_{\text {eff }}$ and $n_{\text {osc }}$, for typical parameters with $m=1$ and $\Delta k / N=1 / 16$. The energy is distributed among many oscillators, and a plateau at $n_{\mathrm{eff}} \approx 0.2$ exists for a long time before the rise to the equilibrium value of $n_{\mathrm{eff}}=0.65$. The $n_{\mathrm{eff}}$ plateau is similar to an effect seen for initial conditions in short wavelengths as in Fig. 2.23. However, the physics is quite different at short wavelengths, where the energy concentrates into a few oscillators, which can be seen by comparing the $n_{\mathrm{osc}}(t)$ plots in Figs. 2.17 and 2.23. In Fig. 2.18 we scale $T_{\text {eq }}$ to the best fit of $\varepsilon / m^{2.75}$ which we will discuss in Sect. 2.8.3.

\subsection{Numerical Results: Relaxation to Equilibrium from High-Frequency Modes}

\subsubsection{Dynamical Studies of Self-Organization into Chaotic Breathers and Their Interaction}

In a systematic study of chaotic breathers (CBs) in a FPU oscillator chain with periodic boundaries, starting from the boundary mode, that has 180 


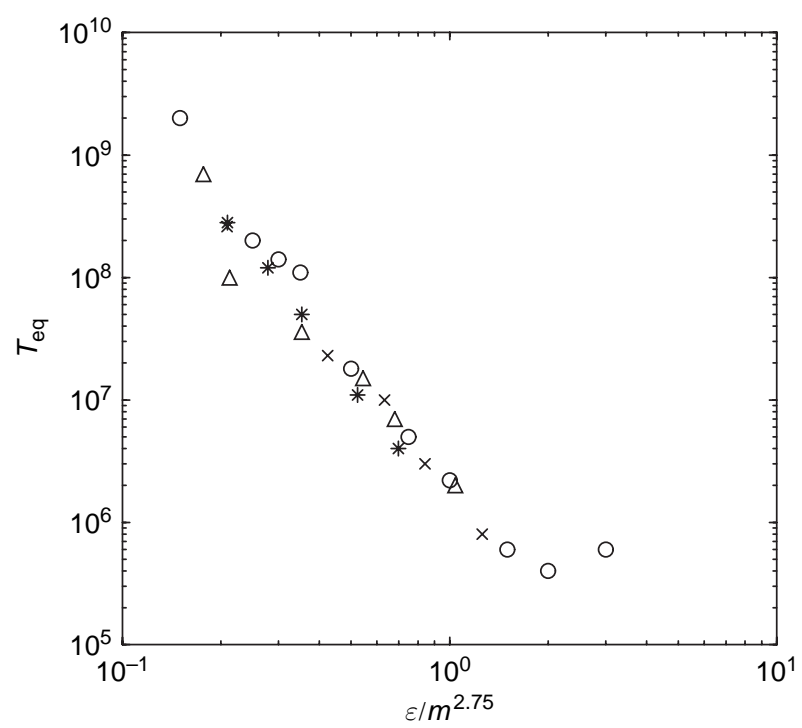

Fig. 2.18. Rescaling of the equipartition time for the $\phi^{4}$ model; each symbol corresponds to a different $\mathrm{m}$-value

phase shift between neighboring oscillators, Cretegny et al. [87] illustrated the dynamics with a three-dimensional presentation first used by Burlakov and Kiselev [83]. The result shown in Fig. 2.19a, with accompanying explanatory figures, shows on a gray intensity scale, the CBs emerging from the fastest growing mode, moving spatially and interacting with one another, with the larger CBs absorbing the smaller ones, until only a single moving $\mathrm{CB}$ remains. Figure 2.19b, c illustrate this with time snapshots. Finally there is a longer time period in which the $\mathrm{CB}$ can continue to grow by taking energy from background modes with similar symmetry, but ultimately decaying to an equipartition state, as seen in Fig. 2.19d. For this study an intermediate value of $\varepsilon \simeq 0.35$ was used. This result is quantified with statistical measures, which we save for the following section.

As already described in Sect. 2.4.6, it is not necessary to start from the $\pi$-mode to obtain results of this nature. In fact, for fixed boundaries there is no exact $\pi$-mode. More generic initial conditions with fixed boundaries were used by Ullmann et al. (2000), obtaining results very similar to Fig. 2.19. For example for $N=128$, choosing $\gamma=120, n=N+1-\gamma=9$ initial peaks, a result close to Fig. 2.19a was obtained, except that there is a competition between the initially fixed number of peaks and the wavenumber of the fastest growing mode. This roughly led to the result that, even for energies for which $n_{m} \equiv N k_{m} / 2 \pi<9$ the number of initial CBs $n_{\mathrm{B}} \geq 9$. They also explored a wide range of initial conditions, finding with $N=128$, that proto-breathers began to form for $\gamma \gtrsim 80$, and became fully formed CBs for $\gamma \gtrsim 100$. They 

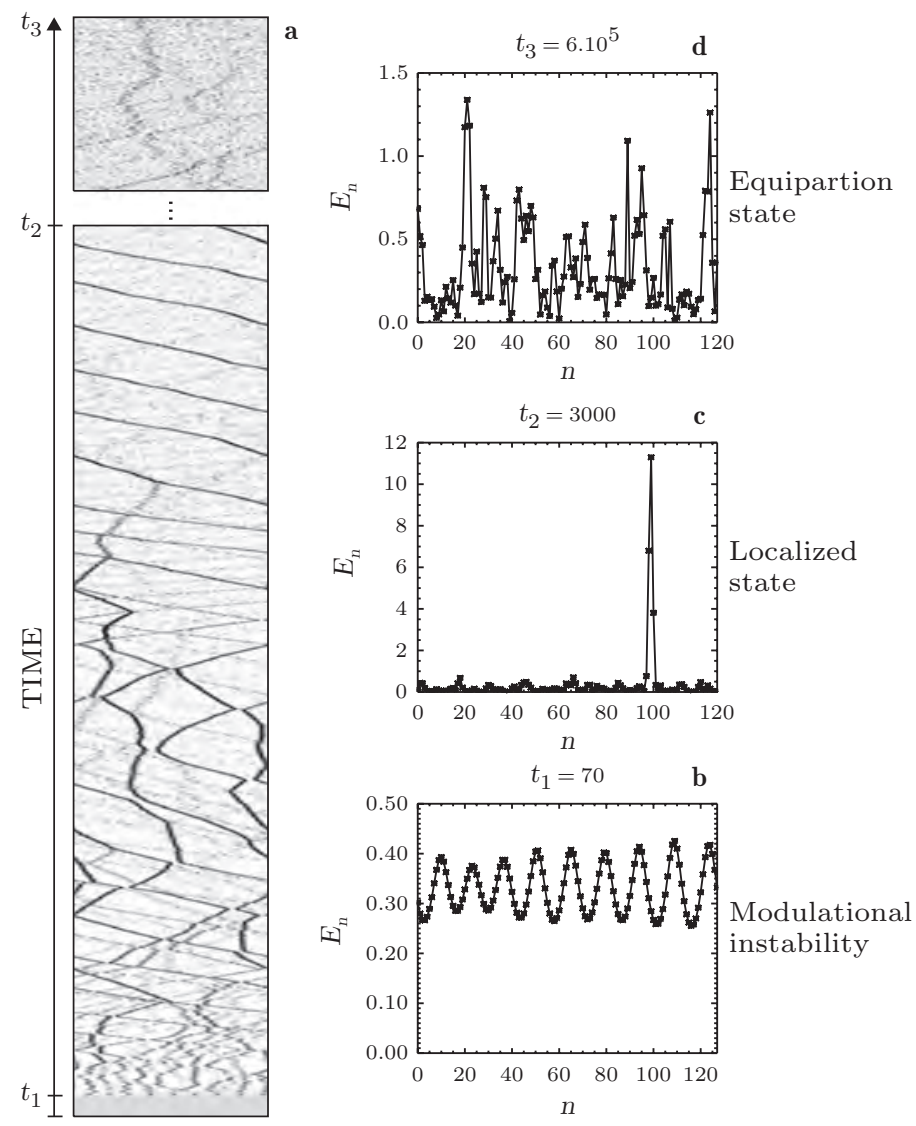

Fig. 2.19. Evolution of the local energy $E_{n}$ along the chain. In panel a, the horizontal axis indicates the position along the chain and the vertical axis corresponds to time (time is going upward). The grey scale goes from $E_{n}=0$ (white) to the maximum $E_{n}$-value (black). The lower rectangle corresponds to $0<t<3000$ and the upper one to $5.994 \times 10^{5}<t<6 \times 10^{5}$. Panels $\mathbf{b}$, c , and $\mathbf{d}$ show the instantaneous $E_{n}$ along the $N=128$ chain at three different times. Note the difference in vertical amplitude

used a particular value of $E=50(\varepsilon=0.39)$. This latter result was obtained by direct observation of the spectrum, and also by the use of macroscopic quantities, as described in the next subsection.

Various numerics of $\mathrm{CB}$ formation and decay were repeated in papers employing the analytic methods using envelope functions, in order to compare analytic formulae with numerical results. For example, for the FPU chain the logarithmic rate of decay of the number of $\mathrm{CBs} 1 / \tau \equiv n_{\mathrm{B}}^{-1}\left(\mathrm{~d} n_{\mathrm{B}} / \mathrm{d} t\right)$ was constant at fixed energy, indicating that the time-scale for coalescence

$$
\tau_{\mathrm{B}} \sim\left(n_{\mathrm{B}} \sigma_{\mathrm{B}} v_{\mathrm{B}}\right)^{-1}
$$


with $\tau_{\mathrm{B}} \propto \tau$, preserved the constancy of the product $\mathrm{CB}$ density $\times \mathrm{CB}$ interaction cross-section $\times \mathrm{CB}$ velocity during the coalescence [92]. At lower initial energies, for the FPU chain, $\tau_{\mathrm{B}} \propto E^{-2}$ [91], while at higher energies $\tau_{\mathrm{B}} \propto E^{-1}$ [88]. These scalings are understood, and the underlying theory will be outlined in Sect. 2.9.

The dynamics of the $\phi^{4}$ chain has some significant differences from the FPU dynamics. Nevertheless, the overall features have much in common. For example, for the lowest mode of the envelope, $n=1(\gamma=128)$, the development of the parametric instability is quite similar for the $\phi^{4}$ and FPU, provided the $\phi^{4}$ energy is a factor of 16 higher to account for the factor of 16 in the nonlinear term, with $E \propto \psi_{m}$ in the energy range considered. The initial nonuniform growth of the instability is shown in the time snapshots in Fig. 2.20, and the coalescence shown in four time snapshots in Fig. 2.21.

By scaling either the energy or the time, the early development of the instability can be obtained reasonably close to that in Fig. 2.20. Similarly, the nearly complete coalescence into a single CB in Fig. 2.21d at $t=5000$, can be roughly compared with the localized state at $t=2000$ from the observation of a residual second breather seen in Fig. 2.19a, and at $t=3000$ in Fig. 2.19c. Note that the faster evolution times in the FPU example reflect reasonably well the effective higher energy.

\subsubsection{Transitions and Time-Scales to Equipartition}

To systematically study the transitions and time-scales for the creation of breathers, their coalescence, and their destruction leading to equipartition,

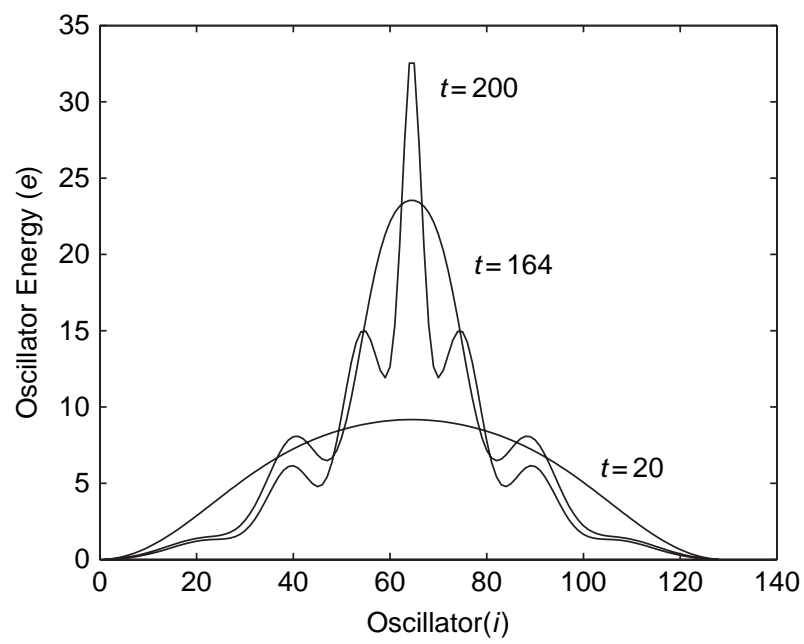

Fig. 2.20. Development of the fastest growing mode from the initial mode $\gamma=$ $128(n=1)$ for the $\phi^{4}$ model 

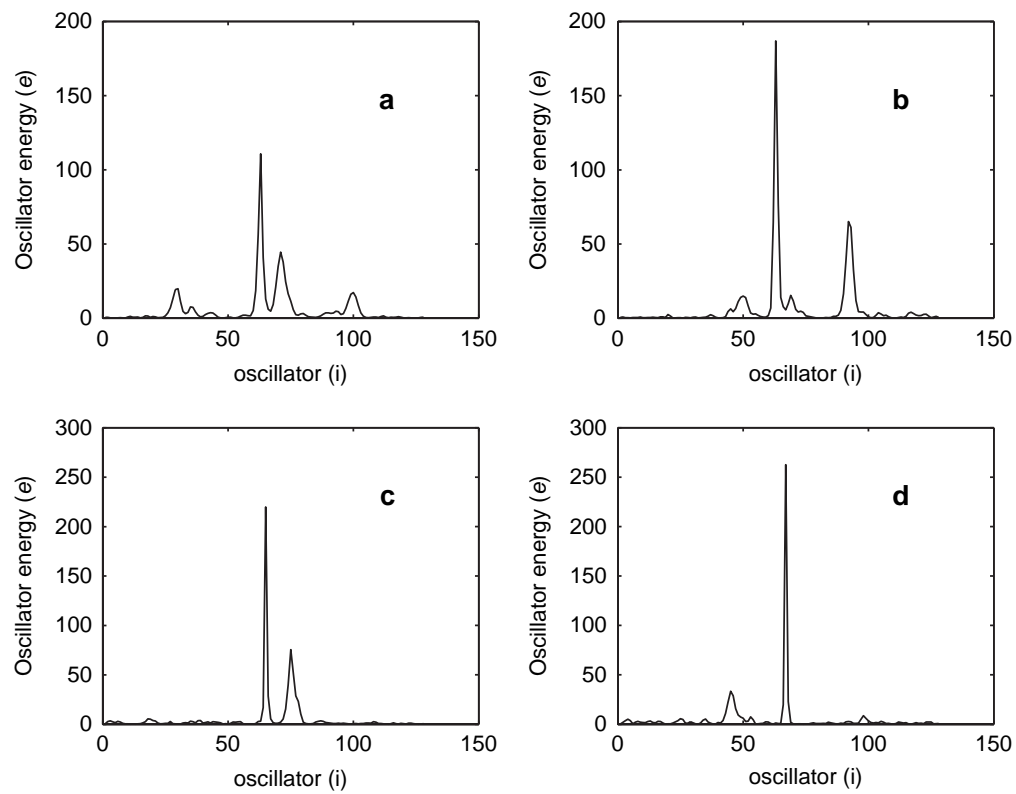

Fig. 2.21. Coalescence of a few chaotic breathers into primarily a single breather fot the $\phi^{4}$ chain: $\mathbf{a} t=300, \mathbf{b} t=1200, \mathbf{c} t=4000$, and $\mathbf{d} t=5000$

macroscopic quantities are most useful. We have introduced these macroscopic quantities in Sect. 2.3.2 and used them to study the evolution from longwavelength modes to equipartition in Sect. 2.5.2. For long-wavelength initial conditions the quantity $n_{\text {eff }}$ is most useful, while $n_{\text {osc }}$ gives little additional information. In contrast, for short-wavelength initial conditions, in which the intermediate $\mathrm{CB}$ state occurs, $n_{\mathrm{osc}}$ is the most useful macroscopic indicator but $n_{\text {eff }}$ also serves a useful function; other macroscopic indicators can also be used. In Fig. 2.22a we plot the evolution of $n_{\mathrm{osc}}(t)$ during the evolution, using a linear time-scale, beginning after the multiple CBs have formed through their coalescence time of about $t \simeq 2 \times 10^{4}$, and then through a longer time interval to approximately $t \simeq 2.5 \times 10^{5}$. Finally, an approximate equipartition is reached at $T_{\text {eq }} \sim 5 \times 10^{5}$, as the breather disintegrates, between $t=2.5 \times 10^{5}$ and $t=5 \times 10^{5}$. It is quite difficult to extract a definite $T_{\text {eq }}$ from $n_{\text {osc }}$, which is most useful for describing the evolution of the CB states. We also present the results for $n_{\text {eff }}$ on a linear time-scale, but over twice the time period, in Fig. $2.22 \mathrm{~b}$, observing that $T_{\text {eq }} \simeq 10^{6}$. The approximate numerical asymptotes are slightly lower than the theoretical values, given in Sect. 2.3.2, which are $n_{\text {osc }}=0.74$ and $n_{\text {eff }}=0.655$.

The overall time dependence is better seen on a scale which is logarithmic in time. We illustrate the full time dependence for the $\phi^{4}$ oscillator chain, in Fig. 2.23, starting from the same initial conditions as in Figs. 2.20 and 2.21. We note here that the asymptotes correspond more closely to the theoretical 

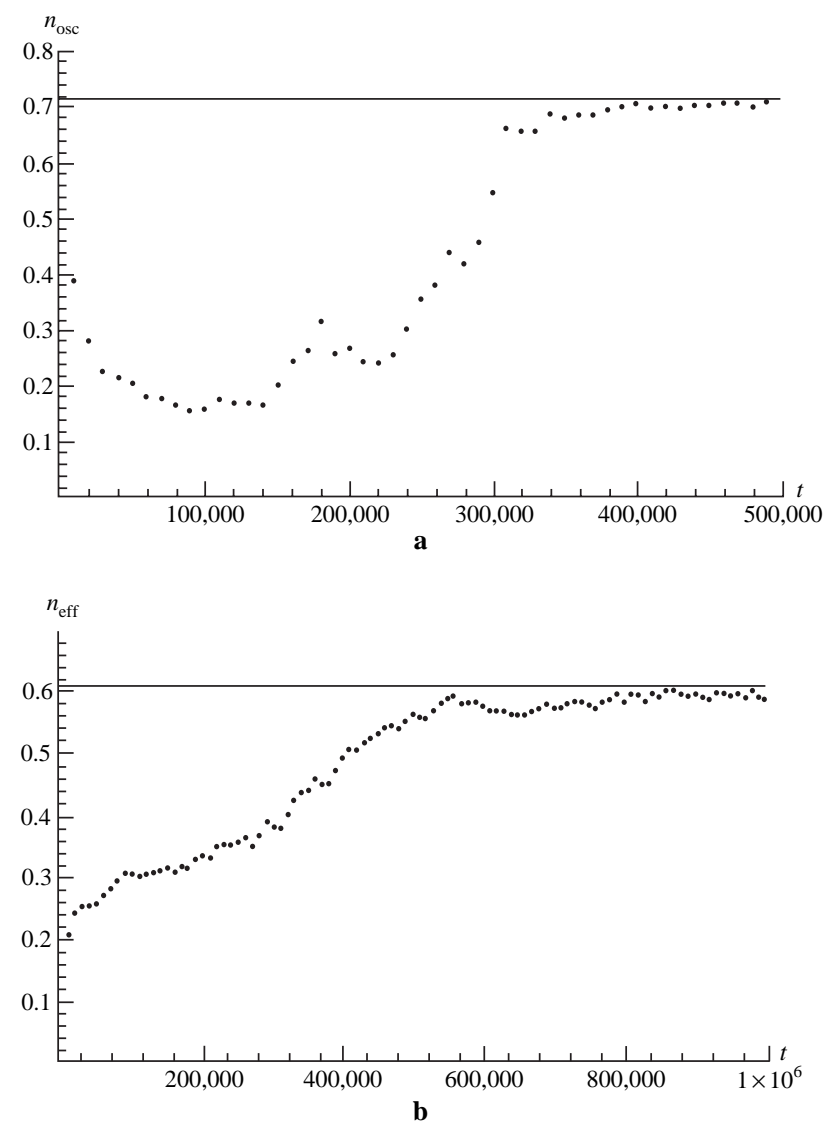

Fig. 2.22. Time dependence of $\mathbf{a} n_{\mathrm{osc}}$ and $\mathbf{b} n_{\mathrm{eff}}$. The horizontal lines are asymptotes

values. Again, most of the relevant information is contained in $n_{\text {osc }}(t)$. The logarithmic dependence of the time-dynamics is seen by estimating that the instability growth is in the range $30 \lesssim t \lesssim 300$, the breather formation and coalescence between $300 \lesssim t \lesssim 3 \times 10^{3}$, and the single breather exists between $3 \times 10^{3} \lesssim t \lesssim 3 \times 10^{4}$. In the final decade the breather is destroyed, leading to equipartition.

The scalings for short-wavelength initial conditions in the regions of lower and higher energy density $\varepsilon$ can be obtained similarly to that in Sect. 2.5.2 for long-wavelength initial conditions. As for long wavelengths there is a transition between two distinct regions of power-law scaling with energy density $\varepsilon$, the lower energy region which has steeper $\varepsilon$-scaling, and the higher energy region with flatter $\varepsilon$-scaling. Returning to the numerics of the FPU, in Fig. 2.24 we plot $n_{\text {eff }}$ vs. normalized time for a series of values of $\varepsilon$, showing that in the lower energy regime $0.33 \leq \varepsilon \leq 1.44$ in Fig. 2.24a, the data 

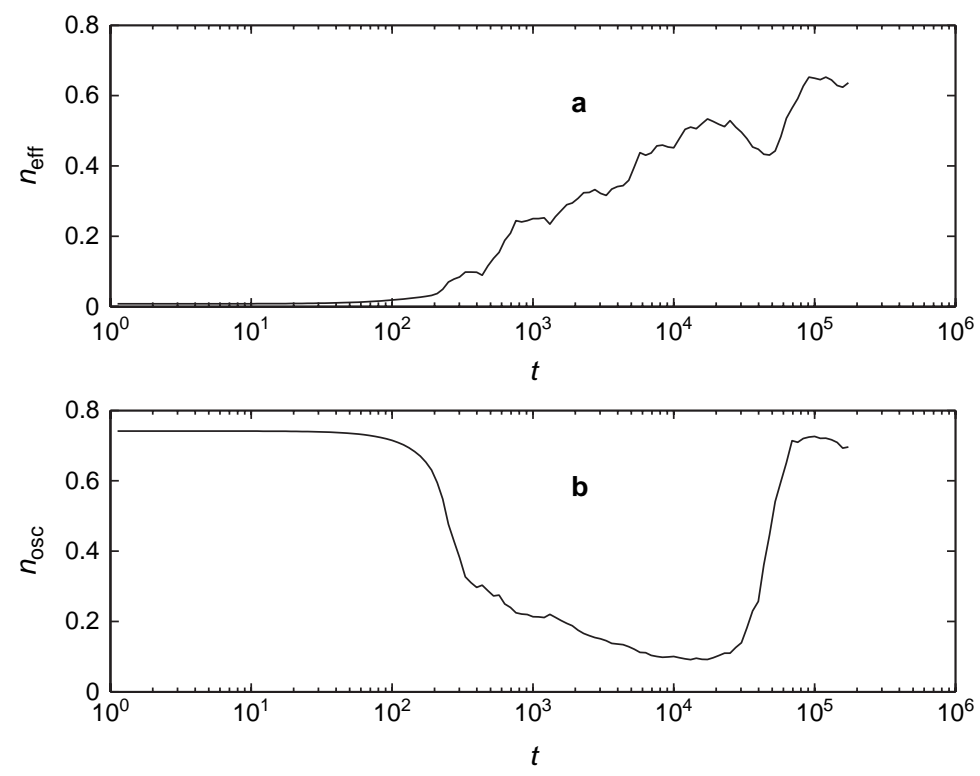

Fig. 2.23. Time dependence of (a) $n_{\text {eff }}$ and (b) $n_{\text {osc }}$ for the $\phi^{4}$ chain. The initial energy density is $\varepsilon=2$ and $\gamma=128(n=1)$

collapses onto a single curve using $\varepsilon^{-2}$ as a normalizing factor. At very high values of $\varepsilon, 10^{3} \leq \varepsilon \leq 10^{6}$ the dynamics has become purely random over the mode space, and the weak normalizing factor $\varepsilon^{-1 / 4}$ is observed (Fig. 2.24b).
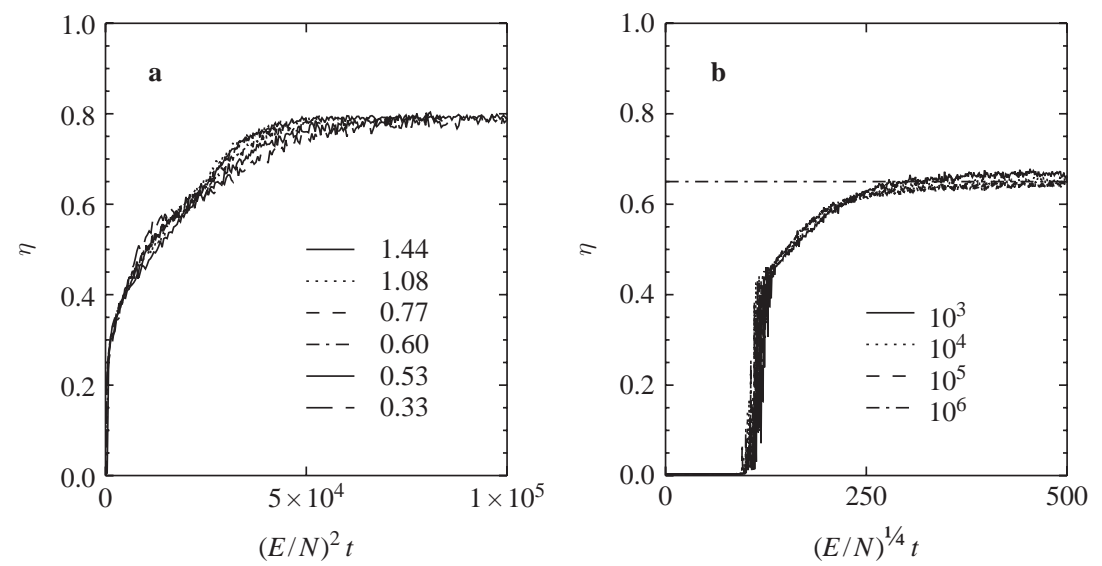

Fig. 2.24. In panel a [resp. b] the evolution of $n_{\text {eff }}(t)$ vs. the rescaled time $t(E / N)^{2}$ (resp. $\left.t(E / N)^{1 / 4}\right)$ is reported for different energy densities. Each curve in (a) [resp. b] corresponds to the average over 20 (resp. 50) different initial conditions for a chain with $N=512$ sites. The dot-dashed line reported in (b) indicates the asymptotic value $n_{\text {eff }} \simeq 0.655$ 


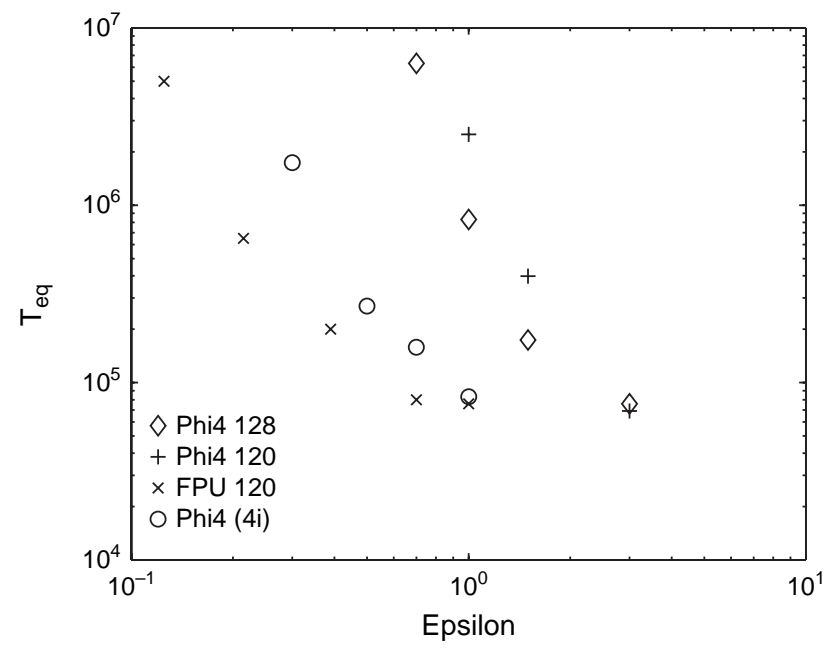

Fig. 2.25. Comparison of the equipartition time for the FPU and the $\phi^{4}$ lattice

The lower $\varepsilon$ scaling, starting from short wave-lengths, is different from the $\varepsilon^{-3}$ scaling obtained in Sect. 2.5.2 from long wave-lengths. Estimates for the scalings, as well as values of $T_{\text {eq }}$ in the various regimes will be presented in Sect. 2.9.2.

Finally, we compare in Fig. 2.25 the times to equipartition, for a few distinct initial conditions of the FPU and $\phi^{4}$ chains at relatively low values of $\varepsilon$, with $N=128$. All values of $T_{\text {eq }}$ were taken at the first crossing (or touching) of $n_{\mathrm{eff}}=0.65$. The slopes become steeper, for both chains and all initial conditions, at the lowest values of $\varepsilon$. This may be associated with the transition to exponential scaling, as previously discussed for low-frequency initial conditions. We also see the basic separation of a factor of 4 in $\varepsilon$ at a given $T_{\text {eq }}$, between the FPU and $\phi^{4}$ results, due to the difference in the nonlinear forces. There are also more subtle differences, discussed in the original paper [94].

\subsection{Numerical Results: Stationary Nonequilibrium Properties}

\subsubsection{Numerical Studies of the Divergence of Heat Conductivity}

As a result of a number of studies of the heat conductivity in FPU and diatomic Toda models the conductivity of long but finite chains diverges as

$$
\kappa(N) \propto N^{\alpha} .
$$

In Table 2.1, we compare the available estimates of the exponent $\alpha$ determined by different authors in various models and conditions. The acronym NEMD refers to nonequilibrium molecular dynamics simulations, where the chain is 
in contact at its boundaries with thermal baths at different temperatures. The heat conductivity is estimated by (2.10). The acronym GK indicates equilibrium simulations, where the chain is at equilibrium and the heat conductivity is computed by the Green-Kubo formula: for an isotropic homogeneous solid made of atoms placed on a regular cubic lattice of volume $V$ in $d$ dimensions, the thermal conductivity $\kappa$ is expressed as a scalar quantity (see [154])

$$
\kappa=\frac{1}{k_{\mathrm{B}} T^{2} d} \lim _{t \rightarrow \infty} \int_{0}^{t} \mathrm{~d} \tau \lim _{V \rightarrow \infty} V^{-1}\langle J(\tau) \cdot J(0)\rangle,
$$

where $k_{\mathrm{B}}$ is the Boltzmann constant; $T$, the temperature of the solid; \langle\rangle , the equilibrium average; and $J$ is the heat current vector.

This formula applies when the integral on the r.h.s. is finite. In a chain of atoms, $J$ is a scalar quantity and a divergent heat conductivity can be signalled by the slow decay in time of the heat current autocorrelation function $\langle J(t) J(0)\rangle \sim t^{-\beta}$ with $\beta<1$. In fact, the integral in (2.129) diverges. In finite chains one expects that an exponential decay eventually sets in, so that simulations should be performed for different chain lengths in order to be sure to pick up the truly asymptotic scaling behavior.

A quantitative comparison with the nonequilibrium measurements, based on the Fourier law (2.10), can be performed by noticing that energy propagates with the constant sound velocity $v_{\mathrm{s}}$. This can be understood by, for example looking at the spatio-temporal correlation function $C(i, t)=\left\langle j_{i}(t) j_{0}(0)\right\rangle$ of the local heat flux plotted in Fig. 2.26 (see [114]). Accordingly, one can turn the time divergence of $\kappa$ as determined from the Green-Kubo formula into a divergence with $N$ by restricting the integral in formula (2.129) to times smaller than the "transit time" $\mathrm{Na} / v_{s}$. This amounts to ignoring all the contributions from sites at a distance larger than $N$. With the above estimate of $C$, one obtains that $\kappa \propto N^{1-\beta}$, i.e. $\alpha=1-\beta$. The latter exponent is the

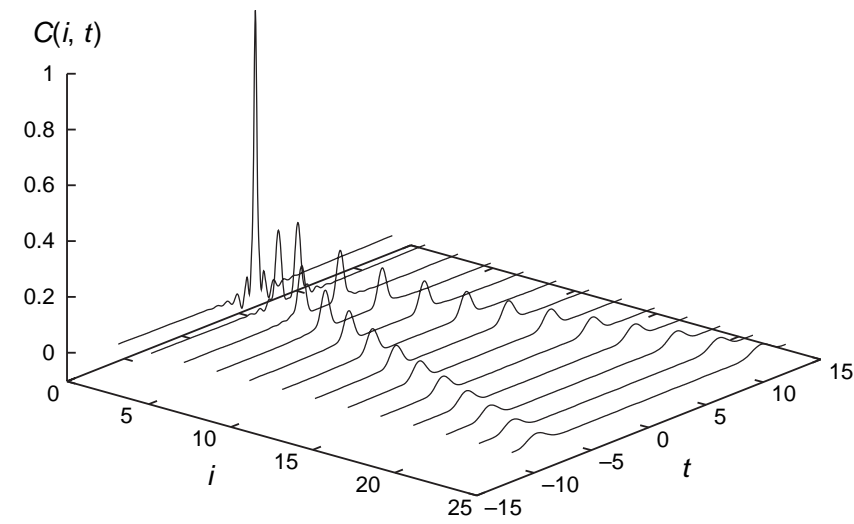

Fig. 2.26. The spatio-temporal correlation function $C(i, t)=\left\langle j_{i}(t) j_{0}(0)\right\rangle$ of the local flux for the FPU- $\beta$ model. Microcanonical simulations, energy density 8.8 
Table 2.1. The estimated exponent $\alpha$ of divergence of the conductivity with size $N$, as obtained from both nonequilibrium molecular dynamics (NEMD) simulations and through Green-Kubo (GK) equilibrium studies. Only the significative digits are reported as given in the quoted References

\begin{tabular}{lccc}
\hline Model & Reference $\alpha$ (NEMD) & $\alpha(\mathrm{GK})$ \\
& & & \\
\hline & & & \\
FPU- $\beta$ & {$[113,114]$} & 0.37 & 0.37 \\
FPU- $\alpha$ & {$[115]$} & $\sim 0.4$ & - \\
Diatomic FPU $r=2$ & {$[117]$} & 0.43 & compatible \\
Diatomic Toda $r=2$ & {$[116]$} & $0.35-0.37$ & 0.35 \\
& {$[117]$} & 0.39 & compatible \\
Diatomic Toda $r=8$ & {$[117]$} & 0.44 & compatible \\
Diatomic hard points & {$[116]$} & 0.35 & - \\
& & & \\
\hline
\end{tabular}

one reported in the last column of Table 2.1. All numerical values there range between 0.35 and 0.44 , suggesting a nontrivial universal behavior. It is also remarkable to notice the overall consistency among the results obtained with different thermostat schemes (ranging from deterministic to stochastic ones).

In order to better appreciate the quality of the divergence rate that can be numerically obtained, in Fig. 2.27 we have plotted the finite-length conductivity $\kappa(N)=J N /\left(T_{+}-T_{-}\right)$vs. the number of particles in the FPU- $\beta$

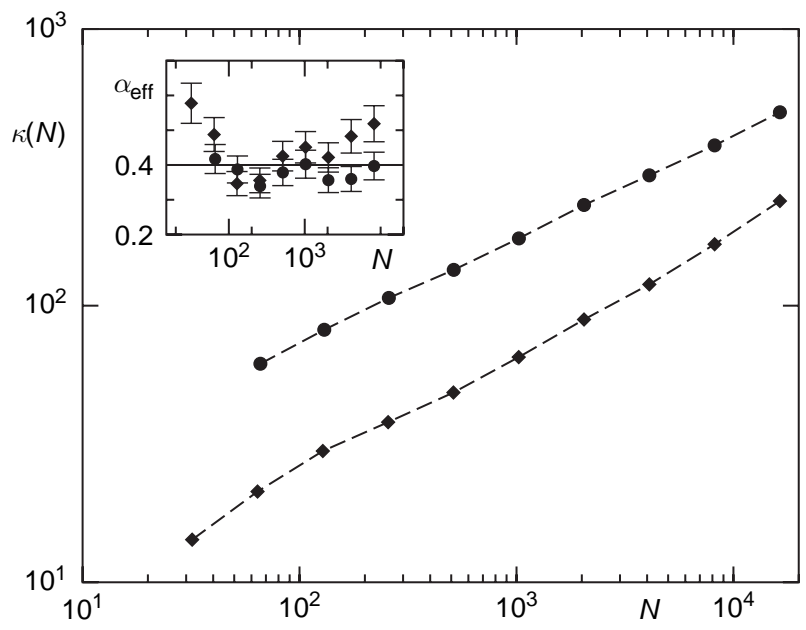

Fig. 2.27. Thermal conductivity of the FPU- $\beta$ model vs. lattice length $N$ for $T_{+}=$ $0.11, T_{-}=0.09$. The inset shows the effective growth rate $\alpha_{\text {eff }}$ versus $N$. Circles and diamonds correspond to free and fixed b.c., respectively 
model for fixed and free boundary conditions. In the inset, one can see that the effective growth rate $\alpha_{\text {eff }}$, which corresponds to the logarithmic derivative of $\kappa(N)$, is basically the same in both cases, despite the clear differences in the actual values of the flux itself. The value of $\alpha_{\text {eff }}$ seems to be quite close to 0.4 , a value which has been predicted by different theoretical approaches (e.g., see [113]).

Once the divergence is clearly established, the next question concerns the universality of the divergence rate. The discussion of this point involves considering a possible dependence on the temperature as well as on the leading nonlinearities [115]. Both questions are addressed in Fig. 2.28, where $\kappa(N)$ is computed in the FPU- $\alpha$ model at a relatively low temperature.

One can see that changes in the temperature gradient, without modifying the average $T=\left(T_{+}+T_{-}\right) / 2$, modify the effective conductivity only at relatively small sizes. Moreover, the two sets of measures corresponding to $\Delta T=0.1$ and 0.02 (triangles and circles, respectively) approach each other for $N$ larger than $10^{3}$. In both cases $\kappa(N)$ increases linearly with $N$ for $N<10^{3}$ and no sizeable temperature gradient forms along the chain. Both facts hint at a weakness of anharmonic effects up to this time/length scales. This is confirmed by the comparison with the results for a pure harmonic chain (with the same setup and same parameters) that exhibit a clean linear growth of $\kappa$ with $N$ (see the solid line in Fig. 2.28) and a few-percent differences in the initial size range. The fact that $\kappa$ is smaller for larger $\Delta T$ can be thus attributed to a stronger boundary scattering that reduces the conductivity. From the inset of

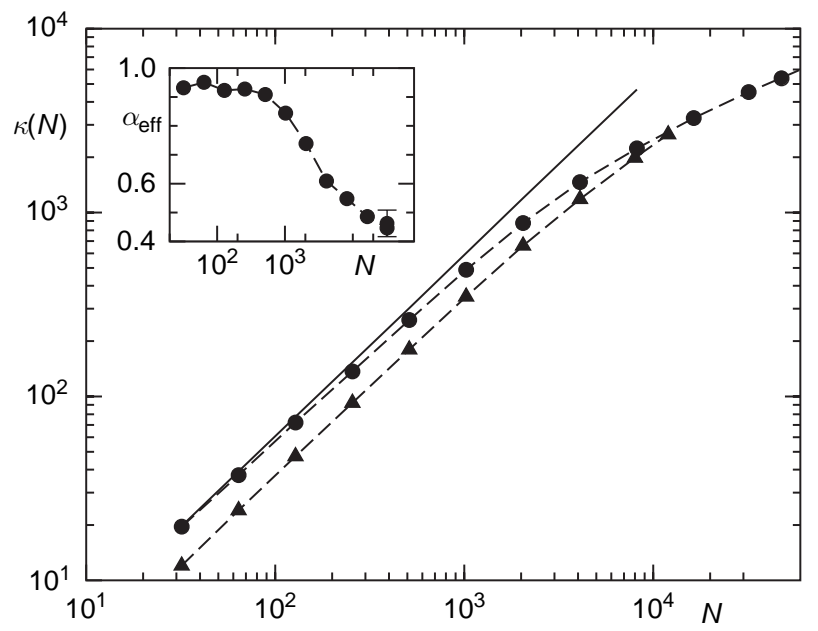

Fig. 2.28. Thermal conductivity of the FPU- $\alpha$ model vs. lattice length $N$. Triangles and circles refer to $\Delta T=0.1$ and $\Delta T=0.02$, respectively. The solid line corresponds to the linear divergence observed in a harmonic chain with the same temperatures. The inset shows the effective divergence rate $\alpha_{\text {eff }}$ vs. $N$ for the data corresponding to full circles 
Fig. 2.28, one can only conclude that for chain sizes up to $\mathrm{O}\left(10^{4}\right)$ the exponent $\alpha$ seems to approach the value 0.4 , although nothing prevents the possibility that it converges to a larger value. In particular, there is evidence that the scaling properties of $\kappa(N)$ in the FPU- $\alpha$ model exhibit much different features with respect to the FPU- $\beta$ model, at least in the explored range of temperatures and sizes. Recent theoretical estimates based on the mode-coupling approach, indicate that, as a matter of symmetry, heat conductivity in the FPU- $\alpha$ and $-\beta$ models should diverge with different values of the exponent in (2.128). Moreover, these values are supposed to represent the two universality classes characterizing the power-law divergence of the heat conductivity for any FPU-like model (including more phenomenological nearest-neighbor potentials, like the Lennard-Jones ones). It must be pointed out that such a theoretical prediction is very difficult to be checked numerically. Typically, finite size/time effects, as those observed in the FPU- $\alpha$ model, are found to last over extremely long integration times and for very large system sizes which do not allow one to confirm or disprove the theoretical expectations. Anyway, a power-law divergence of the heat transport coefficient is always present in such a class of models, although in finite systems the value of the exponent $\alpha$ varies significantly with temperature and size. It is worth mentioning that this could be a relevant point for what concerns possible comparison with experiments, performed on almost-one-dimensional systems, like carbon nanotubes or polymers.

Finally, we want to discuss the role of the boundary resistance in connection with the temperature dependence of conductivity. In fact, an interesting application of (2.128) has been proposed with reference to the FPU- $\beta$ model [155]. There, it has been empirically found that the bulk conductivity scales with $N$ and $T$ as

$$
\kappa \simeq\left\{\begin{array}{ll}
1.2 N^{\alpha} T^{-1} & (T \sim 0.1) \\
2 N^{\alpha} T^{1 / 4} & (T>50)
\end{array} .\right.
$$

According to kinetic theory, the conductivity can also be expressed as $\kappa=$ $\ell v_{\mathrm{S}} C_{v}$, where $\ell$ is the mean free path of phonons. Since $C_{v}$ and $v_{\mathrm{s}}$ are almost constant and of order 1 in a wide temperature range, $\ell \sim \kappa$. Hence, at low temperatures the boundary jumps dominate the thermal profile up to the size $N_{*}$ that can be estimated according to (2.130). At low temperatures this effect is very strong with $N_{*} \sim(2 \varepsilon / T)^{(1 / 1-\alpha)}$, while smaller boundary resistances are found at large temperatures, with $N_{*} \sim\left(2 \varepsilon T^{1 / 4}\right)^{(1 / 1-\alpha)}$, where $\varepsilon$ is the energy density.

\subsubsection{Force Laws That Predict Classical Heat Conductivity:Coupled-Rotors, Ding-a-Ling and Klein-Gordon Chains}

A simple example of a classical-spin one-dimensional model with nearest neighbor interactions has a potential 


$$
V(x)=1-\cos x,
$$

i.e. is a chain of $N$ coupled pendula. It has been extensively studied $[151,153$, 156] as an example of a chaotic dynamical system that becomes integrable both in the low- and high-energy limits, when it reduces to a harmonic chain and to free rotors, respectively. In the two integrable limits, the relaxation to equilibrium slows down very rapidly for most of the observables of thermodynamic interest (e.g., the specific heat: see $[151,156]$ ). As a consequence, the equivalence between ensemble and time averages is established over accessible time-scales only inside a limited interval of the energy density $\varepsilon$. Here, we focus our attention mainly on heat conduction in the strongly chaotic regime.

It has been shown that, contrary to the expectations, this model exhibits a finite conductivity in spite of the existence of an acoustic branch in its spectrum in the harmonic limit $[157,159]$. Simulations have been performed for $T_{+}=0.55, T_{-}=0.35$, and chain lengths ranging from $N=32$ to 1024 with fixed boundary conditions and Nosé-Hoover thermostats [157]. The equations of motion have been integrated with a fourth order Runge-Kutta algorithm and a time step $\Delta t=0.01$. The results, reported in Fig. 2.29 clearly reveal a convergence to a value of $\kappa$ approximately equal to 7 (see the circles). The dotted line is the best fit with the function $a+b / N$ and indicates a constant $\kappa$ for $N \rightarrow \infty$. This is the first system where normal heat conduction was convincingly ascertained in the absence of a local potential and was confirmed by calculating an exponential decay of heat flux correlation appearing in the Green-Kubo formula (2.129).

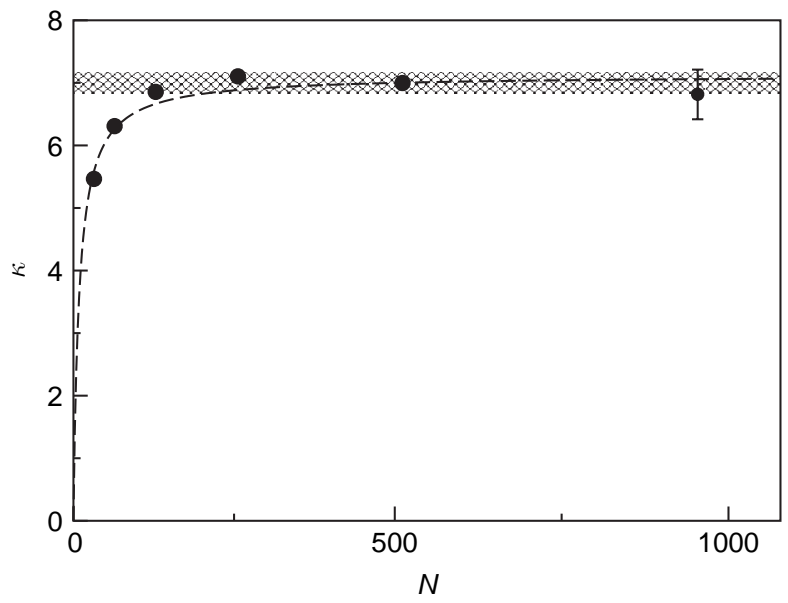

Fig. 2.29. Conductivity $\kappa$ vs. chain length $N$ as obtained from nonequilibrium molecular dynamics. Circles correspond to the rotator model with temperatures $T_{+}=0.55$ and $T_{-}=0.35$. The dashed line represents the best fit with the function $a+b / N$. The shaded region represents the uncertainty about the conductivity on the basis of the Green-Kubo formula 
One explanation of the striking difference in the transport behavior exhibited by the model compared to, for instance, the FPU models is to notice that the pair potential $V\left(q_{i+1}-q_{i}\right)$ possesses infinitely many equivalent valleys. As long as $\left(q_{i+1}-q_{i}\right)$ remains confined to the same valley, there is no reason to expect any qualitative difference with, for example, the FPU- $\beta$ model. Phase slips (jumps of the energy barrier), however, may very well act as localized random kicks, that contribute to scattering of the low-frequency modes, thus leading to a finite conductivity. In order to test the validity of this conjecture, one can study the temperature dependence of $\kappa$ for low temperatures when jumps across barriers become increasingly rare. The data plotted in Fig. 2.30 indicate that the thermal conductivity behaves as $\kappa \approx \exp (\eta / T)$ with $\eta \approx 1.2$. The same scaling behavior is exhibited by the average escape time $\tau$ (see triangles in Fig. 2.30) though with a different $\eta \approx 2$. The latter behavior can be explained by assuming that the phase slips are the results of activation processes. Accordingly, the probability of their occurrence is proportional to $\exp (-\Delta V / T)$, where $\Delta V$ is the barrier height to be overcome. The behavior of $\tau$ is thus understood, once we notice that $\Delta V=2$. In the absence of phase slips, the dependence of the conductivity on the length should be the same as in FPU- $\beta$ model, i.e. $\kappa \approx N^{\alpha}$, with $\alpha \approx 0.4$. In the presence of phase slips, it is natural to expect that the conductivity is limited by the average distance $\bar{N}$ between consecutive phase slips. Under the further assumption of a uniform distribution of the slips, their spatial and temporal separation has to be of the same order, thus implying that $\kappa(T)$ exhibits the same divergence as $\tau$ for $T \rightarrow 0$, though with a different rate $\kappa \approx \exp [\alpha \Delta V / T]$. Therefore, at least

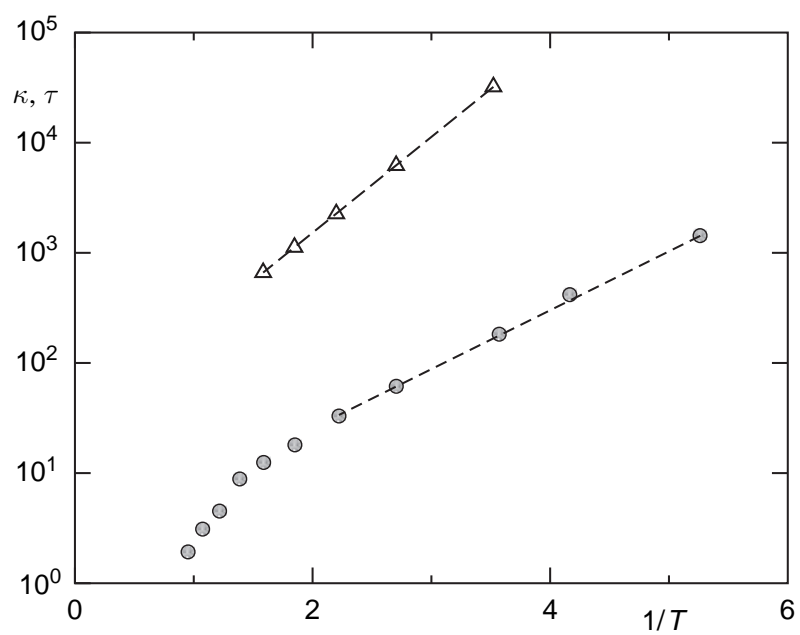

Fig. 2.30. Thermal conductivity $\kappa$ vs. the inverse temperature $1 / T$ in the rotor model (open circles). Triangles correspond to the average time separation between consecutive phase slips in the same system 
on a qualitative level, one can indirectly confirm that phase slips are responsible for the normal heat transport. However, there is a discrepancy between the observed and the expected value of the exponent $\eta$ (1.2 vs. 0.8). We now consider the class of models whose Hamiltonian contains a local "substrate" potential $U\left(q_{i}\right)$. This means that translational invariance breaks down and the total momentum is no longer a constant of the motion. Accordingly, the dispersion relation is such that $\omega(k) \neq 0$ for $k=0$.

The so-called "ding-a-ling" model was first introduced as a toy model for a one-dimensional plasma [158]. It can refer in different contexts to : (i) a set of identical charge-sheets embedded in a fixed neutralizing background; (ii) a system of harmonic oscillators with the same frequency and equilibrium positions sitting on a periodic lattice and undergoing elastic collisions that exchange their velocities. Notice that in the low-energy limit, it reduces to a set of independent harmonic oscillators with equal frequency (no dispersion). A modified version of this model, where the harmonic oscillators (say the evennumbered particles) alternate with free particles of the same (unit) mass, was later introduced for studying heat transport [119]. The free particles are only constrained to lie between the two adjacent oscillators. The Hamiltonian can be symbolically written as

$$
\mathcal{H}=\frac{1}{2} \sum_{l}^{N}\left[p_{l}^{2}+\omega_{l}^{2} q_{l}^{2}\right]+\text { "hard point core" }
$$

where $\omega_{l}=\omega$ for even $l$ and zero otherwise. A common feature of this class of models is that within collisions the motion of the particles can be determined analytically so that the basic requirement is the computation of the occurrence times of the collision events. Therefore, the dynamics naturally reduces to a discrete mapping. For an isolated system (e.g., a chain with periodic boundary conditions) the dynamics depends only on the dimensionless parameter $\varepsilon /(\omega a)^{2}$ where $\varepsilon$ is the energy per particle and $a$ the lattice spacing. The dynamical behavior of the model was studied by fixing $\varepsilon=1$ and changing $\omega$ [119]. When $\omega$ and $N$ were large enough the dynamics was found to be strongly chaotic and soliton-like pulses are sufficiently attenuated [160]. This renders the model a good candidate to check the validity of Fourier's law.

The Fourier law was first confirmed with the performance of a series of non-equilibrium simulations, where the freely moving end-particles were put in contact with two Maxwellian reservoirs. The average flux $J$ was then computed by summing the amounts of energy $\delta E$ exchanged with one of the reservoirs in all collisions during the simulation time. The average temperature gradient was estimated with a linear fit (to get rid of boundary effects). By evaluating the thermal conductivity as a function of the lattice length up to $N=18$ for $T_{+}=2.5, T_{-}=1.5$ and $\omega=1$, it was concluded that $\kappa(N)$ attains a constant limiting value for $N>10$ (see Fig. 2.31). After having established the existence of a finite value of the transport coefficient, the value of $\kappa$ was compared with the result of linear response theory, using a Green- 


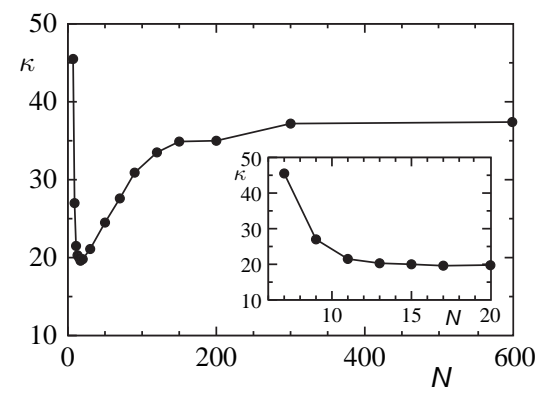

Fig. 2.31. Thermal conductivity of the ding-a-ling model. Size dependence of $\kappa$ for $\omega=1$ and $\varepsilon=1.5$ (from [162]). In the inset an expanded view is presented in the range of sizes considered in [119]

Kubo formula (see [161]). In summary, all these studies provided a convincing numerical evidence that the heat conductivity is finite in this model. In particular, it was shown that the energy transport is diffusive for large values of $\omega$ (typically, larger than 10), while the linear response estimate was found to agree with numerical simulations. Much later, these results were essentially confirmed by a detailed series of simulations with longer chains and in a wider parameter range of the "ding-a-ling" model [162]. However, the temperature profile exhibits a nonlinear shape (see Fig. 2.32) different from the typical linear temperature profile predicted by the Fourier law.

Careful numerical studies performed on the original Dawson model [161] have confirmed the validity of Fourier's law in a wide temperature range. Besides direct nonequilibrium simulations with Maxwellian thermostats and the Green-Kubo formula, these studies implemented an efficient transient method that allowed them to explore the high-temperature regime $(T>3)$, where, because of the nearly integrable dynamics, a slow convergence of the averages with time and/or size is observed. In the low-temperature limit $(T<0.1)$ it was also proved that the heat conductivity vanishes as $\exp (-1 / 4 T)$, in agreement with numerical results. Numerical evidence of finite thermal conductivity

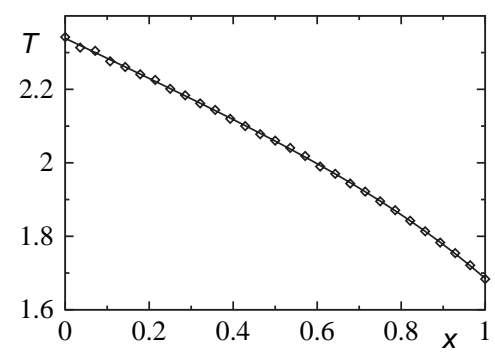

Fig. 2.32. Temperature profile for the ding-a-ling model, $\omega=2$ (see [163]) 
was also found in a modified ding-a-ling model where the harmonic potential is replaced by a gravitational one [163].

The Klein-Gordon lattices are another important class of models with a substrate potential, where the interparticle potential is harmonic:

$$
\mathcal{H}=\sum_{l=1}^{N}\left[{\frac{p_{l}}{2 m}}^{2}+U\left(q_{l}\right)+\frac{1}{2} C\left(q_{l+1}-q_{l}\right)^{2}\right] .
$$

The first and most complete study of the transport problem in this class of models has been carried out for the Frenkel-Kontorova potential [164].

$$
U(x)=-U_{0} \cos \left(\frac{2 \pi x}{a}\right) .
$$

The model can be interpreted as a chain of either coupled particles in an external periodic field or torsion pendula subject to gravity. In the latter case $a=2 \pi$ and $q_{l}$ represents the angle with respect to the vertical direction: it can be read as the discretized (and nonintegrable) version of the well-known sine-Gordon field equation.

Besides energy, the dynamics admits a further conserved quantity, the winding number $\mathcal{P}$, which is an integer defined by the boundary condition $q_{l+N}=q_{l}+a \mathcal{P}$. In the particle interpretation, $\mathcal{P}$ represents the number of potential wells, while for the pendula it can be viewed as the degree of built-in twist in the system. Thermal conductivity was computed numerically in the general case of nonvanishing winding number, with three different methods: (i) attaching two heat baths; (ii) through the Green-Kubo formula; (iii) by adding an external field. All the methods give consistent results and clearly indicate that the thermal conductivity is finite. These results were later confirmed by further numerical studies [165], which investigated the dependence of the transport coefficient on the lattice length (for $\mathcal{P}=0$ ). Similar conclusions were drawn for a more general version of the Frenkel-Kontorova model with an anharmonic inter-site potential [166].

In order to illustrate the type of behavior observed in this class of models Fig. 2.33 shows some data for the $\phi^{4}$ chain

$$
U(x)=\frac{a}{2} x^{2}+\frac{b}{4} x^{4} .
$$

In panel (a), we present a case of fast convergence to a small $\kappa$ value for a single-well potential; panel (b) refers instead to a low-temperature regime characterized by large thermal conductivity.

Evidence of a finite conductivity for the case $a=0$ has been reported in $[167,168]$. Two further examples were analyzed, the sine-Gordon and bounded single-well potentials:

$$
U(x)=\cosh x-1 \quad, \quad U(x)=\frac{1}{2}\left(1-\operatorname{sech}^{2} x\right)
$$

representatives of the classes of hard and soft anharmonicity, respectively. In both cases there is numerical evidence for finite thermal conductivity [169]. 

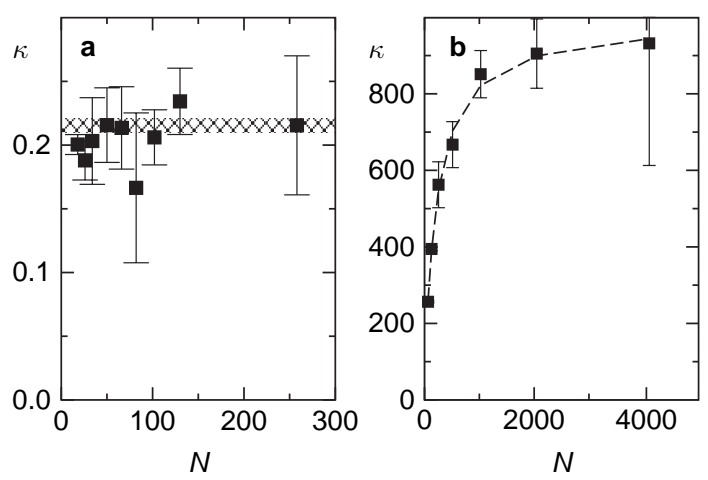

Fig. 2.33. Thermal conductivity vs. chain length in $\phi^{4}$ chains with Nosé-Hoover thermostats. Panel a refers to the single well case $[a=b=1$ in (2.135)] the results have been obtained for $C=1, T_{+}=8$, and $T_{-}=6$. The shaded region represents the value obtained from the Green-Kubo formula with its statistical uncertainty. Panel $\mathbf{b}$ refers to the double-well case $(a=-1, b=1)$ for an average temperature $T=0.37$ and a temperature difference 0.002 . The dashed line is just a guide for the eyes

\subsection{Analytical Calculations and Estimates: Scaling Estimates for $\lambda$ and for $T_{\text {eq }}$ from Low Frequencies}

\subsubsection{Random Matrix Approximation in the Strong Mode Overlap Regime}

The numerical computation of the energy density $(\varepsilon=E / N)$ scaling of the largest Lyapunov exponent for the FPU- $\beta$ model has put in evidence the existence of a crossover between two scaling laws: $\lambda(\varepsilon) \propto \varepsilon^{2}$ at low-energy density, and $\lambda(\varepsilon) \propto \varepsilon^{2 / 3}$ at larger $\varepsilon$ values [60], reaching on an asymptotic value at large energy of $\lambda \propto \varepsilon^{1 / 4}$.

This transition has been called [64] the Strong Stochasticity Threshold (SST), and it has been ascribed to the (smooth) transition between a regime of weak chaos and a regime of strong chaos. Even if the system is prepared far from energy equipartition, corresponding to the crossover energy density $\varepsilon_{\mathrm{c}}$ there is a transition between slow and fast relaxation to equipartition; the relaxation time approximately constant for energy densities greater than $\varepsilon_{\mathrm{c}}$, but steeply growing with decreasing the energy density below this value. Although the relaxation phenomenology depends on the details of the initial conditions, they always group into two families separated by $\varepsilon_{c}$. For example, below $\varepsilon_{\mathrm{c}}$ the initial excitation of high-frequency normal modes results in a slower relaxation to equipartition with respect to the initial excitation of lowfrequency modes, but this situation is reversed above $\varepsilon_{\mathrm{c}}$ [64]. We can estimate the $\varepsilon$-scaling of $\lambda$ to a transition from weak to strong chaos from a random matrix approximation (RMA) for the tangent dynamics, which approximately 
accounts for the high-energy scaling of $\lambda$, whereas the extrapolation of such a scaling law to lower energy increasingly overestimates the actual values of $\lambda[53,64]$.

The RMA assumes the absence of correlations: for a flow this can be assumed if the sampling time is not too short and if the dynamics itself mimics a true random process. It then appears reasonable to consider as strongly chaotic the dynamical regime where the RMA accounts for the $\lambda(\varepsilon)$ scaling and to consider weakly chaotic the dynamical regime where the RMA largely overestimates $\lambda(\varepsilon)$. In $[53,64]$ it has been suggested that in the strongly chaotic regime phase space diffusion occurs across resonances (and is therefore a fast diffusion entailing a fast mixing) thanks to the coalescence of the stochastic layers, generated near the resonant surfaces. At variance, below the SST, the widths of the stochastic layers could be such that the resonance overlap ceases - or is considerably reduced - so that phase space diffusion should be constrained to occur along tortuous paths, along resonances, in a definitely less efficient fashion; this qualitative picture has been given an interesting quantitative confirmation in [170]. Here the relaxation times of the weakly chaotic regime measured in [53] have been analytically estimated with a very good accuracy by means of a theoretical approach closely resembling the so-called quasi-linear theory of diffusion in plasma physics, a theory of slow diffusion applicable when chaos is not fully developed.

The numerical integration of a Hamiltonian flow consists in recursively computing the symplectic coordinate transformation $\left[q_{i}(t), p_{i}(t)\right] \rightarrow\left[q_{i}(t+\right.$ $\left.\tau), p_{i}(t+\tau)\right]$

$$
\begin{aligned}
& q_{i}(t+\tau)=q_{i}(t)+\tau p_{i}(t) \\
& p_{i}(t+\tau)=p_{i}(t)+\tau F_{i}[q(t+\tau)]
\end{aligned}
$$

where $F_{i}=-\partial V(q) / \partial q^{i}$ are the forces and $q=\left(q_{1}, \ldots, q_{N}\right)$. The Jacobian matrix

$$
\underline{J}(\Omega)=\left(\begin{array}{ll}
\mathbb{I} & \tau \mathbb{I} \\
\tau \Omega & \mathbb{I}+\tau^{2} \Omega
\end{array}\right)
$$

of the discretized flow is symplectic, i.e

$$
\underline{J}^{\mathrm{T}} \underline{E} \underline{J}=\underline{E}
$$

where

$$
\underline{E}=\left(\begin{array}{ll}
0 & \mathbb{I} \\
-\mathbb{I} & 0
\end{array}\right) .
$$

Here $\mathbb{I}$ is the $N \times N$ identity matrix and $\Omega$ is the Hessian of the potential part of the Hamiltonian: $\Omega_{i j}=-\partial^{2} V(q) / \partial q_{i} \partial q_{j}$ and $\tau$ is a discretization time (for instance the time integration step). $\underline{J}$ is a $2 N \times 2 N$ symplectic matrix which maps a vector $\xi(t)$ tangent to the flow at time $t$ into a vector $\xi(t+\tau)$, that is: $\xi(t+\tau)=\underline{J}(t) \xi(t)$. Using Oseledec's multiplicative theorem [171], an approximation of $\lambda$ is given by 


$$
\lambda=\lim _{n \rightarrow \infty} \frac{1}{n \tau} \ln \left\langle\left[\frac{\xi^{\mathrm{T}}(0)\left[\prod_{j=1}^{n} \underline{J}^{\mathrm{T}}(q(j \tau))\right] \cdot\left[\prod_{k=1}^{n} \underline{J}(q(k \tau))\right] \xi(0)}{\xi^{\mathrm{T}}(0) \cdot \xi(0)}\right]^{1 / 2}\right\rangle .
$$

Now, if with a suitable time sampling the dynamics mimics a good random process then both $\Omega$ and $\underline{J}$ can be considered as random matrices. For the FPU- $\beta$ model, the matrix $\Omega$ can be expressed as the sum of a tridiagonal constant matrix $\Omega_{0}$ and of an implicitly time-dependent tridiagonal matrix $\widetilde{\Omega}$. The matrix elements of $\Omega$ contain combinations of terms like $\left(q_{i+1}-q_{i}\right)^{2}$. In the random matrix approximation, the hypothesis of $\delta$-correlation in time is made for the fluctuating part $\widetilde{\Omega}$ of $\Omega$, i.e. $\left\langle\widetilde{\Omega}_{i j}(k \tau) \widetilde{\Omega}_{i j}(l \tau)\right\rangle=\left(\gamma_{i j} / \tau\right) \delta_{k l}$. The average $\langle\cdot\rangle$ in (2.139) is carried over different realizations of the random matrix process. The theoretical computation of (2.139) yields [53]

$$
\lambda \sim \tau^{2 / 3} \gamma^{1 / 3}
$$

where $\gamma$ is defined as

$$
\gamma=\frac{1}{N} \sum_{i=1}^{N}\left\langle\left[\left(q_{i \pm 1}-q_{i}\right)^{2}-\left\langle\left(q_{i \pm 1}-q_{i}\right)^{2}\right\rangle\right]^{2}\right\rangle .
$$

By computing a statistical ensemble average of $\gamma(\varepsilon)$, one finds $\gamma(\varepsilon) \sim \varepsilon^{2}$ in an intermediate $\varepsilon$-range, as outlined below. Thus, from (2.140), $\lambda \sim \varepsilon^{2 / 3}$ if $\varepsilon$ is not too large and if $\tau$ is independent of $\varepsilon$. We remark that this result is not asymptotic. The microcanonical measure should be used, but the canonical measure can equivalently work at large $N$. The canonical configurational partition function

$$
Z_{\mathrm{C}}^{(N)}=\left[\Gamma\left(\frac{1}{2}\right)\left(\frac{\eta \beta}{2}\right)^{-1 / 4} \exp \left(\frac{\alpha^{2} \eta}{8 \beta}\right) D_{-1 / 2}\left(\alpha \sqrt{\frac{\eta}{2 \beta}}\right)\right]^{N}
$$

where $\alpha$ is a dummy parameter multiplying the harmonic part of the FPU potential, $D_{-1 / 2}$ is a parabolic cylinder function, $\eta=1 / T\left(k_{\mathrm{B}}=1\right)$ is the inverse of the average kinetic energy per particle which is, within a good approximation, proportional to $\varepsilon$ thus $\eta \simeq 1 / \varepsilon$. The ensemble average $\langle\gamma\rangle$ is then given by

$$
\langle\gamma\rangle=Z_{\alpha=1}^{-1}\left[-\frac{4}{\eta}\left(\frac{\partial Z}{\partial \beta}\right)_{\alpha=1}\right]-\left[Z_{\alpha=1}^{-1}\left(-\frac{2}{\eta}\right)\left(\frac{\partial Z}{\partial \alpha}\right)_{\alpha=1}\right]^{2},
$$

and using the asymptotic approximation $D_{-1 / 2}(x) \sim \exp \left(-x^{2} / 4\right) x^{-1 / 2}(1-$ $\left.3 x^{2} / 8+\cdots\right)$ that holds good at $x \gg 0$ one immediately gets

$$
\langle\gamma\rangle(\varepsilon) \sim \varepsilon^{2} .
$$

At high $\varepsilon$, where the above expansion for $D_{-1 / 2}$ worsens, the exponent 2 is lowered and consequently also the $2 / 3$ exponent of $\lambda(\varepsilon)$ is lowered. In fact, 
the numerical results for $\lambda(\varepsilon)$ and the analytic results worked out by means of the geometric approach, outlined in Sect. 2.4.7 and applied to the FPU- $\beta$ model in Sect. 2.8.2, give at high $\varepsilon$ the asymptotic scaling $\lambda(\varepsilon) \sim \varepsilon^{1 / 4}$.

In conclusion, the transition from the low-energy density scaling $\lambda(\varepsilon) \sim \varepsilon^{2}$ to the scaling $\lambda(\varepsilon) \sim \varepsilon^{2 / 3}$ is attributed to the transition from two different regimes of chaoticity because the $\lambda(\varepsilon) \sim \varepsilon^{2 / 3}$ scaling is accounted for by the RMA for the tangent dynamics at moderately high $\varepsilon$ values.

\subsubsection{Geometric Calculation of $\lambda$ for FPU- $\beta$}

A completely analytical computation of $\lambda(\varepsilon)$ has been performed - in the $N \rightarrow \infty$ limit - for the FPU $\beta$-model using the geometric method sketched in Sect. 2.4.7. As we shall see below, the agreement is strikingly good. Particularly noticeable is the fact that the analytic values of $\lambda$ check with the numerical ones within errors of few percent in a range of six orders of magnitude both in $\varepsilon$ and $\lambda$, with no adjustable parameters. The analysis follows [60] and has been reviewed in [42].

The geometric quantities appearing in (2.125), that is $\Omega_{0}$ and $\sigma_{\Omega}$, written in the Eisenhart metric assume the simple form

$$
\begin{aligned}
& \Omega_{0}=\left\langle k_{R}\right\rangle_{\Sigma_{E}}=\frac{1}{N}\langle\Delta V\rangle_{\Sigma_{E}}, \\
& \sigma_{\Omega}^{2}=\left\langle\delta^{2} k_{R}\right\rangle_{\Sigma_{E}}=\frac{1}{N}\left(\left\langle(\triangle V)^{2}\right\rangle_{\Sigma_{E}}-\langle\triangle V\rangle_{\Sigma_{E}}^{2}\right),
\end{aligned}
$$

where $\triangle V$ is the euclidean Laplacian of the potential function $V(q)$.

Now, the microcanonical ensemble averages of $K_{\mathrm{R}}(q)$ and of its variance can be computed in terms of the corresponding quantities in the canonical ensemble as follows. The canonical configurational partition function $Z(\eta)$ is given by

$$
Z(\eta)=\int \mathrm{d} q \mathrm{e}^{-\eta V(q)},
$$

where $\mathrm{d} q=\prod_{i=1}^{N} \mathrm{~d} q_{i}$. The canonical average $\left\langle K_{\mathrm{R}}\right\rangle_{\text {can }}$ of the Ricci curvature $K_{\mathrm{R}}$ follows as

$$
\left\langle K_{\mathrm{R}}\right\rangle_{\text {can }}=[Z(\eta)]^{-1} \int \mathrm{d} q K_{\mathrm{R}}(q) \mathrm{e}^{-\eta V(q)}
$$

From this average, we can obtain the microcanonical average of $K_{\mathrm{R}},\left\langle K_{\mathrm{R}}\right\rangle_{\Sigma_{E}}$, in the following (implicit) parametric form [172]

$$
\left.\begin{array}{l}
\left\langle K_{\mathrm{R}}\right\rangle_{\Sigma_{E}}(\eta)=\left\langle K_{\mathrm{R}}\right\rangle_{\mathrm{can}}(\eta) \\
\varepsilon(\eta)=\frac{1}{2 \eta}-\frac{1}{N} \frac{\partial}{\partial \eta}[\log Z(\eta)]
\end{array}\right\} \rightarrow\left\langle K_{\mathrm{R}}\right\rangle_{\Sigma_{E}}(\varepsilon)
$$


Note that (2.146) is strictly valid only in the thermodynamic limit; at finite $N,\left\langle K_{\mathrm{R}}\right\rangle_{\Sigma_{E}}(\eta)=\left\langle K_{\mathrm{R}}\right\rangle_{\mathrm{can}}(\eta)+\mathrm{O}\left(\frac{1}{N}\right)$.

Contrary to the computation of $\left\langle K_{\mathrm{R}}\right\rangle$, which is insensitive to the choice of the probability measure in the $N \rightarrow \infty$ limit, computing the fluctuations of $K_{\mathrm{R}}$, i.e., of $\left\langle\delta^{2} K_{\mathrm{R}}\right\rangle=\frac{1}{N}\left\langle\left(K_{\mathrm{R}}-\left\langle K_{\mathrm{R}}\right\rangle\right)^{2}\right\rangle$, by means of the canonical or microcanonical ensembles yields different results. The relationship between the canonical-i.e. computed with the Gibbsian weight $\mathrm{e}^{-\beta H}$ - and the microcanonical fluctuations, is given by the Lebowitz-Percus-Verlet formula [172]

$$
\left\langle\delta^{2} K_{\mathrm{R}}\right\rangle_{\Sigma_{E}}(\varepsilon)=\left\langle\delta^{2} K_{\mathrm{R}}\right\rangle_{\mathrm{can}}(\eta)-\frac{\eta^{2}}{c_{V}}\left[\frac{\partial\left\langle K_{\mathrm{R}}\right\rangle_{\mathrm{can}}(\eta)}{\partial \eta}\right]^{2}
$$

where

$$
c_{V}=-\frac{\eta^{2}}{N} \frac{\partial\langle H\rangle_{\mathrm{can}}}{\partial \eta}
$$

is the specific heat at constant volume and $\eta=\eta(\varepsilon)$ is given in implicit form by the second equation in (2.146).

For the FPU- $\beta$ model, the Ricci curvature, written in the Eisenhart metric, simply reads

$$
K_{\mathrm{R}}=2 N+6 \beta \sum_{i=1}^{N}\left(q_{i+1}-q_{i}\right)^{2} .
$$

Note that $K_{\mathrm{R}}$ is always positive. By taking advantage of the analytically known form of $Z(\eta)$ for the one-dimensional FPU- $\beta$ model, one can exactly compute the analytic expressions of the microcanonical averages of (2.146) and (2.147) in the $N \rightarrow \infty$ limit. With $\Omega_{0}$ and $\sigma_{\Omega}$ one computes $\tau$ and, substituting $\Omega_{0}(\varepsilon), \sigma_{\Omega}(\varepsilon)$, and $\tau(\varepsilon)$ into (2.125), we finally get $\lambda(\varepsilon)$, which is reported in Fig. 2.34. As anticipated at the beginning of the present Section, the analytic values of $\lambda$ are in excellent agreement with the numerical ones.

Other systems for which good results have been obtained are coupled rotators [60], as shown in Fig. 2.35, classical XY Heisenberg models [173], $\varphi^{4}$ models [141, 174], and the "mean-field" XY model [175], although some adjustments are necessary in these cases, mainly related with the nontrivial topology of the mechanical manifolds.

\subsubsection{Estimates of Time to Equipartition with Strong Arnold Diffusion}

In the energy regime in which the diffusion from a chaotic low-frequency driving resonance can efficiently couple to a high-frequency resonance, i.e. when the ratio of the driving to the driven frequencies $\omega_{\mathrm{B}} / \delta \omega_{h} \sim 1$, an analytic estimate of the equipartition time can be made [58]. The estimate predicts a scaling $T_{\text {eq }} \propto \varepsilon^{-3}, \varepsilon \equiv E / N$, independent of $E$ and $N$ separately, for the FPU- $\beta$ chain. The method has also been applied to estimate the equipartition time for energy placed initially in short-wavelength (high-frequency) modes, 


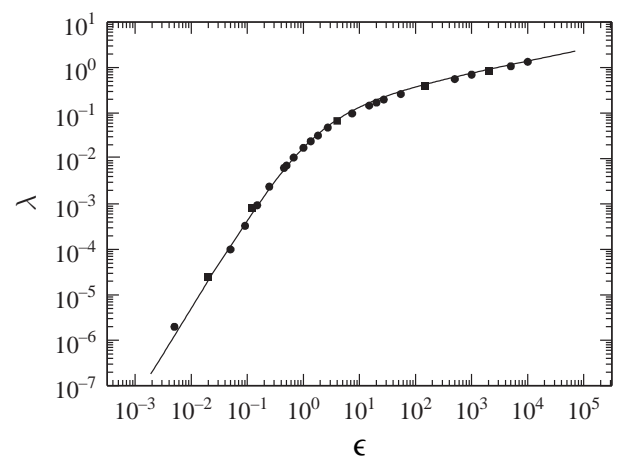

Fig. 2.34. Lyapunov exponent $\lambda$ vs. energy density $\varepsilon$ for the FPU- $\beta$ model with $\beta=$ 0.1 . The continuous line is the theoretical computation according to (2.125), while the circles and squares are the results of numerical simulations with $N$ respectively equal to 256 and 2000

obtaining a scaling $T_{\text {eq }} \sim \varepsilon^{-2}$ [92]. These scalings agree with those found numerically, and furthermore give values of $T_{\text {eq }}$ well within a factor of 10 of the numerical values. The basic method of making the estimation is outlined below.

The assumption is that there is an effective number of driving modes $\delta k$, dependent on the total energy, that transfer energy efficiently through the Arnold diffusion mechanism, i.e. satisfying (2.50). Considering that $E_{\gamma} \simeq$ $E / \delta k$, then the number of interacting modes $\delta k$ is, from $(2.50)$,

$$
\delta k=\mu \beta E
$$

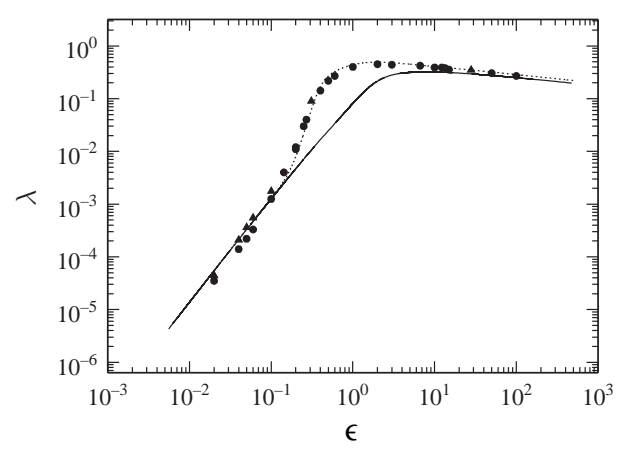

Fig. 2.35. Lyapunov exponent $\lambda$ vs. energy density $\varepsilon$ for the one-dimensional $X Y$ model. The continuous line is the theoretical computation according to (2.125), while full circles, squares, and triangles are the results of numerical simulations with $N$, respectively, equal to 150,1000 , and 1500 . The dotted line is the theoretical result where the value of $\Omega_{0}$ entering (2.125) has been corrected in order to empirically account for nontrivial configuration space topology 
where $\mu$ is of order unity. The procedure is then to calculate the rate that energy is extracted from the modes containing energy, to be distributed among all modes, with an integration over time until all modes have equal energy (at equipartition). Since the energy is spread among many modes, we can take the energy to be primarily in the linear part of the Hamiltonian. To be specific we consider the case of transfer from long wavelengths to short wavelengths.

The change in the linear energy $E_{i}=(1 / 2)\left(P_{i}^{2}+\omega_{i}^{2} Q_{i}^{2}\right)$ of a driving lowfrequency mode $i$, can be calculated from the action-angle form of the Hamiltonian by taking the derivative with respect to the angle $\theta_{i}$

$$
\begin{aligned}
\frac{\mathrm{d} E_{i}}{\mathrm{~d} t}= & \left(\frac{-2 \beta}{N+1}\right) \omega_{i} \sum_{j, h_{1}, h_{2}} B\left(i, j, h_{1}, h_{2}\right) \\
& \sqrt{\omega_{i} \omega_{j} \omega_{h 1} \omega_{h 2} I_{i} I_{j} I_{h} I_{h 2}} \sin \theta_{i} \operatorname{ang}\left(j, h_{1}, h_{2}\right),
\end{aligned}
$$

where $\operatorname{ang}\left(j, h_{1}, h_{2}\right)$ is a product of cosines of the angle variables. The notation $h 1$ and $h 2$ explicitly indicates that the energy transfer occurs between a lowfrequency beat oscillation and a high-frequency mode difference oscillation through the Arnold diffusion mechanism. In the above equation, the summation is over indices $j, h_{1}$, and $h_{2}$ for a given $i$. The only terms to transfer energy to high-frequency modes are the ones where $j=i$, since then the product of the two low-frequency angles does not have a fast phase associated with it. Additionally, the selection rule requires that $B=0$ unless

$$
2 i+h_{1}+h_{2}=2 N+2,
$$

which reduces the index to a single sum. This result for a single low-frequency mode $i$ is an estimate for the average energy decay rate, which, from (2.151) is

$$
\frac{\mathrm{d} E_{i}}{\mathrm{~d} t}=-\left(\frac{2 \beta}{N}\right) \omega_{i} \frac{\beta E}{2 \pi} E_{i} E_{h}(t),
$$

where $\omega_{i}=\pi i / N$. Since $\delta k$ low-frequency modes, assumed to have energy, couple to $\delta h$ high-frequency modes with $\delta k=\delta h$, the cross couplings imply each high-frequency mode is coupled on average to $\delta k / 4$ low-frequency modes. The effect of the interaction of the phases between driving modes, when more than one driving term exists, has not been studied. The simplest assumption is that the effect from each low-frequency driving resonance is independent. Setting $\omega_{i}=\beta E / N(i=\delta h / 2=\beta E / \pi)$, and dividing by $E_{i}$, we obtain, an average, for each mode in the package

$$
\frac{\mathrm{d} E_{i}}{E_{i}}=-\frac{\beta}{\pi}\left(\frac{\beta E}{N}\right)^{2} E_{h}(t) \mathrm{d} t .
$$


Integrating (2.154) in time, with $E_{i}(t)$ varying from $E / \delta k$ at $t=0$ to the equipartition value $E / N$ at the final time $T_{\text {eq }}$ one obtains

$$
\ln \left(\frac{N}{\delta k}\right) \simeq\left(\frac{\beta}{\pi}\right)\left(\frac{\beta E}{N}\right)^{2} \int_{0}^{\mathrm{T}} E_{h}\left(t^{\prime}\right) \mathrm{d} t^{\prime},
$$

The final step in the approximation is to estimate the value of $\int_{0}^{\mathrm{T}} E_{h}\left(t^{\prime}\right) \mathrm{d} t^{\prime}$ at $T=T_{\text {eq }}$, a time of "near-equipartition." The quantity $E_{h}(t)$ appears in an integral, so that its exact form is not required. For a diffusive process in which the amplitudes of the modes increase with $t^{1 / 2}$, we might expect the mode energies to increase linearly with $t, E_{h}(t) \simeq(t / T)(E / N)$, such that the time dependence is independent of $N$. This is found to be approximately true, numerically, over most of the evolution to near-equipartition. Other forms of the time dependence of $E_{h}$ lead to only small numerical differences. Evaluating the integral with the assumption of linear time dependence of $E_{h}(t)$ we obtain

$$
T_{\mathrm{eq}} \simeq \frac{2 \pi}{(\beta E / N)^{3}} \ln \left(\frac{\pi}{2 \beta E / N}\right) .
$$

The numerical coefficient is only a rough estimate. Equation (2.156) exhibits a basic scaling of $T \propto(N / E)^{3}$. The scaling has been checked numerically, by rescaling the time as shown in Fig. 2.13. This scaling was also found by plotting the time to reach $n_{\text {eff }} / N=0.4$ against $E / N$. However, it was also pointed out that a "best fit" stretched exponential also fit the data (see Fig. 2.14). One can also compare the magnitude of $T_{\mathrm{eq}}$ in (2.156) with the numerics. From Fig. 2.15, taking $E / N=0.05$ we find $T_{\text {eq }} \sim 10^{7}$. Considering the many approximations, this value is reasonably close to the value of $T_{\text {eq }} \simeq 3 \times 10^{7}$ obtained from (2.156).

The same calculation method can be modified to determine the scaling and estimate the value of $T_{\text {eq }}$ from high-frequency mode initial conditions. The beat frequency in this case is the difference between the breather nonlinear frequency and the linear-mode frequencies, which are again proportional to $\beta \varepsilon$ as in the above analysis. However the high frequency is $\omega_{i} \simeq 2$ and thus a factor of $\beta \varepsilon$ is removed from the drive, which gives the basic scaling of $T_{\text {eq }} \propto(\beta \varepsilon)^{-2}$. This scaling was confirmed numerically, and the theoretic and numerical values were again in reasonable agreement for high frequencies $[92$, 93, 94], as summarized in Sect. 2.9.2.

As previously noted, on-site potentials, as in the $\phi^{4}$ chain, involve additional complications, and the comparisons were correspondingly less definitive. In particular, in [65] the $m$-scaling was estimated from the calculation to be related to the $\varepsilon$ scaling by the factor $\varepsilon / m^{2}$, in contrast to the numerical result found in Fig. 2.18 which suggested $\varepsilon / m^{2.75}=$ const. to produce the best scaling. 


\subsection{Analytical Calculations and Estimates: Scaling Estimates from High Frequencies}

\subsubsection{Estimate of Coalescence into Single Chaotic Breather}

It is not possible to make a complete quantitative calculation for the very complicated process of chaotic breather coalescence; however, arguments have been made for the FPU chain [91, 92] and for the $\phi^{4}$ chain [94] that predict the scaling of the coalescence time with energy density. We review the main arguments without details. The coalescence time-constant $\tau_{\mathrm{B}}$ is estimated from the standard description

$$
\tau_{\mathrm{B}}=\frac{1}{n_{\mathrm{B}} \sigma_{\mathrm{B}} v_{\mathrm{B}}}
$$

where $n_{\mathrm{B}}=k_{m} N / 2 \pi$ is the initial number of breathers formed earlier in time by the instability, which scales with $\psi_{m}, v_{\mathrm{B}}$ is scaled by application of the virial theorem to a moving breather and $\sigma_{\mathrm{B}}$ is scaled from the Born approximation. For the FPU chain, the asymptotic scalings at small and large amplitude for each factor, are

$$
n_{\mathrm{B}} \propto\left\{\begin{array} { l } 
{ \psi _ { m } } \\
{ \text { const. } }
\end{array} \quad v _ { \mathrm { B } } \propto \left\{\begin{array}{l}
\psi_{m} \\
\text { const. }
\end{array}, \quad \sigma_{\mathrm{B}} \propto\left\{\begin{array}{l}
\psi_{m}^{2}, \text { small amplitude } \\
\psi_{m}^{4}, \text { large amplitude }
\end{array}\right.\right.\right.
$$

such that, taking the product in (2.157), we obtain a coalescence rate scaling with amplitude

$$
\tau_{\mathrm{B}} \propto \psi_{m}^{4}
$$

essentially independent of the amplitude. Since numerically one can use energy as our independent variable, $\psi_{m}$ can be related to $E$ through the breather equilibria, as given in the above references, which vary from low to high energy, giving $\psi_{m} \propto E^{1 / 2}$ and $\psi_{m} \propto E^{1 / 4}$, for the FPU- $\beta$ at low and high energies, respectively. (The $\phi^{4}$ proportionalities are similar but not exactly the same). Using the proportionalities above, for the FPU- $\beta$ in (2.159), we obtain

$$
\tau_{\mathrm{B}} \propto\left\{\begin{array}{l}
E^{-2} \text { small amplitude } \\
E^{-1} \text { large amplitude }
\end{array} .\right.
$$

The results in (2.160) have been nummerically verified at the higher amplitude in [88] with some indication also of the steeper low amplitude scaling. The $\phi^{4}$ chain has slightly different scaling and also some additional complications as discussed in the original paper [94]. It was also noted in that paper that $n_{\mathrm{B}}$ is not necessarily determined by the fastest growing unstable mode, for all initial conditions. In fact, most of the FPU numerics were performed with an initial mode $\gamma=120$, for which $n_{\mathrm{B}} \simeq 9$, initially, independent of $\psi_{m}$. However, since most initial conditions were taken at sufficiently large $\psi_{m}$ such that $n_{\mathrm{B}}$ is approximately constant, as calculated from the fastest growing mode, the scaling was the same for the two cases. 


\subsubsection{Estimate of Time to Equipartition}

As already mentioned in Sect. 2.8.3, an estimate of the $T_{\text {eq }}$ can be made, starting from high frequencies, using the same general procedure, as used there, for initial low frequencies. The main differences are

(i) That the stochastic beat frequency $\Omega_{\mathrm{B}}$ is given by

$$
\Omega_{\mathrm{B}}=\omega_{\mathrm{B}}-\omega_{h},
$$

the difference between the breather frequency $\omega_{\mathrm{B}}$ and the high freequencies $\omega_{h}$, where $\omega_{h} \simeq 2$, and from $(2.89), \omega_{\mathrm{B}} \simeq\left(4+6 \beta \psi_{m}^{2}\right)^{1 / 2}$. The beat frequencies can be calculated analytically, but are quite complicated over the range of energies explored numerically. A good approximation was found to be

$$
\Omega_{\mathrm{B}} \simeq 0.2 \beta E_{\mathrm{B}}
$$

which was used in the calculation [92].

(ii) For faster than exponential Arnold diffusion the CB energy $E_{\mathrm{B}}$ needs to be sufficiently large that

$$
\Omega_{\mathrm{B}} / \delta \omega_{l}>1
$$

with the minimum $\delta \omega_{l}=\pi / N$ at low frequencies. Since this value is much larger than the minimum $\delta \omega_{h}$, the energies required to obtain stochastic diffusion from high to low frequencies is also larger, as seen numerically.

(iii) However, this is partly cancelled by the fact that $\omega_{\mathrm{B}}$ in $(2.162)$ does not depend on a mode frequency that varies as $N^{-1}$. Because of the absence of the additional factor the resultant scaling is $T_{\text {eq }} \propto \varepsilon^{-2}$, rather than the $T_{\text {eq }} \propto \varepsilon^{-3}$ scaling for low energies. We do not repeat the calculation, which is, after these modifications, similar to that presented in Sect. 2.8.3, with the final result for the FPU- $\beta$ chain [92]

$$
T_{\mathrm{eq}} \simeq \frac{80 \pi}{5}\left(\frac{\beta E}{N}\right)^{-2} .
$$

The scaling of $\varepsilon^{-2}$ has been checked numerically [88] with the absolute numerical time approximately a factor of 5 longer that that predicted by (2.164).

A similar calculation has been performed for the $\phi^{4}$ chain [94]. There are some additional complications, and the resultant scaling is not precisely $\varepsilon^{-2}$ but varies with somewhat steeper scaling for the lowest values of $\varepsilon$. We have already seen these results numerically in Sect. 2.6.2. These difficulties have been considered, resulting in a more complicated estimate for the scaling of $T_{\text {eq }}(\varepsilon)$ than that given in (2.164) for the FPU- $\beta$ chain.

Another question that arises is whether for high-frequency initial conditions there is a clear transition, with increasing energy from $T_{\text {eq }} \propto \varepsilon^{-2}$ to weaker $\varepsilon$ scalings as found for low-frequency initial conditions. Clearly there is a change of scaling at higher specific energy, as observed numerically in an extreme case, shown in Fig. 2.24. There is also numerical evidence for such transitions for both the FPU- $\beta$ and $\phi^{4}$ chains in Fig. 2.25. The transition has 
not been investigated in detail, to determine whether it is relatively sharp, i.e. a SST, or more diffuse. Since $\tau_{\mathrm{B}} \propto \varepsilon^{-1}$ and $T_{\text {eq }} \propto \varepsilon^{-2}$, one would expect some type of significant transition at an $\varepsilon$ sufficiently high that the two time-scales cross, such that a CB is not formed. Extrapolating the data in, for the FPU- $\beta$ chain with $E$ varied and $N=128$, the cross-over occurs at $\varepsilon \simeq 4.5(\beta=0.1)$. But this range of $\varepsilon$ has not been numerically investigated. For the more complicated $\phi^{4}$ chain there is no numerical evidence for a SST, but again there have been no computations in the $\varepsilon$-range for which such a transition might be expected.

\subsection{Conclusions and Final Comments}

It often happens that the failure of numerical or experimental results to substantiate theoretical predictions leads to the productive development of new physics. So it was with the Fermi-Pasta-Ulam (FPU) problem in which a one-dimensional chain of masses was connected to its nearest neighbors by nonlinear restoring forces (2.1). Enrico Fermi initially suggested the problem as a method of confirming his prediction that the nonlinearity would lead to equipartition among the degrees of freedom, therefore leading to a dynamical underpinning for statistical mechanics. However, the coupled differential equations, numerically integrated on a first-generation large digital computer, gave the seemingly contrary result that the energy initially placed in the lowest of the harmonic normal modes resulted in periodic (or near periodic) energy oscillations among the first few modes (see Fig. 2.1). The oscillations were soon explained from perturbation theory as the nonlinear beating among neighboring modes, but the fundamental question of whether equipartition would eventually be reached was not answered. The early attempts to analyze the dynamics led to better ways of employing perturbation theory and to better understanding of nonlinear mode coupling.

The most celebrated result, following the lack of confirmation of statistical properties of the FPU dynamics, was the development of soliton theory. In an attempt to understand the apparent stability of recurrences Norman Zabusky and Martin Kruskal found a Taylor expansion of the discreteness, valid for long-wavelength modes, that recovered partial differential equations different from the original nonlinear spring which produced the discretized chain of oscillators. The resulting equations are the Korteveg-de Vries (KdV) equation for the FPU- $\alpha$ chain with cubic nonlinearity, and the modified Kortevegde Vries $(m K d V)$ equation for the FPU- $\beta$ chain with quartic nonlinearity. Nonlinear equations of this and related types had been known to have stable traveling solutions where the dispersion and nonlinearity balance to produce constant amplitude and propagation velocity. An arbitrary initial condition, such as the lowest linear mode on the FPU- $\beta$ chain breaks up initially into a set of structures each having a steady traveling solution with its own velocity. Remarkably, these structures are sufficiently stable that they pass through one 
another without breaking up, and the observed recurrences can be interpreted in terms of their superpositions. But these results did not improve on the best perturbation calculations and are clearly limited to long-wavelength (lowfrequency) modes by the approximations which led to (2.6). Partial differential equations have an infinite number of freedoms, such that general integrability from arbitrary initial conditions requires an infinite number of invariants of the motion. The real excitement came when it was shown that such an infinite set of invariants exists and the new field of soliton theory and applications was born. A single initial nonlinear mode solution of the $\mathrm{mKdV}$ equation is found to become unstable as the energy is increased. A linearization around the nonlinear structure predicted the unstable wave numbers and growth rates, and showed that the values correspond to the observed mode growth for the same discretized structure on the FPU- $\beta$ chain (see Sect. 2.4.3). The result in which one soliton decomposes into a finite number is not inconsistent with general soliton theory, but the instability gives us insight into the dynamics that leads to chaotic motion and, ultimately, to equipartition.

Another important consequence of the numerical and theoretical work, which attempted to both explain the original results and extend them to other energies, initial conditions, and force laws, was that of relating highdimensional oscillator chain dynamics to low-dimensional chaos theory. The development of KAM theory by Kolmogorov, Arnold, and Moser showed that despite the lack of global integrals of the motion in generic systems of two or more degrees of freedom (which in fact, motivated Fermi's initial FPU study), for small perturbations from integrable systems most of the phase space could still be regular. But countering this, another result by V. I. Arnold showed that generic systems of three or more degrees of freedom had stochastic resonance channels in the phase space that could reach close to any portion of the phase space. Furthermore, a heuristic understanding of high-dimensional systems indicated that the fraction of the phase volume that is stochastic increases with increasing number of freedoms. Investigation of the rates of diffusion through the stochastic web indicated that the "Arnold diffusion" was normally exponentially slow in the perturbation parameter, but could become large if the perturbation became large (see [15], Chap. 6). The results suggested that, for $N$ freedoms and with fixed perturbation strength (fixed energy density), near equipartition would probably be obtained with increasing $N$ (the thermodynamic limit), but the time-scales might be exponentially long and unapproachable computationally.

In addition to the low-dimensional theory that contributed to the understanding of high-dimensional Hamiltonian dynamics, the development of statistical measures were essential to the numerical investigations that elucidated the system behavior. Although various methods have been useful, the most important have been the calculation of Lyapunov exponents for the separation of neighboring trajectories, and the information entropy which qualitatively computes the number of modes taking part in the dynamics (see Sects. 2.1.6 and 2.3). To obtain equipartition it is necessary for the dynamics to be ergodic. 
Mixing, which implies ergodicity, is a property that holds if all trajectories are on the average exponentially separating (positive KS entropy). This cannot be exactly true for a divided phase space, and thus it might be qualitatively stated that, if almost all of the space has positive KS entropy, then for practical purposes equipartition will be reached. Because the criterion depends on exponentially diverging trajectories, an obvious set of quantities to be examined are the Lyapunov exponents, giving the separation of trajectories, with positive values indicating exponential separation of trajectories, i.e. chaos. The numerical calculation of Lyapunov exponents has been used extensively to test for chaotic motion, as we have reviewed in Sect. 2.5.

Although the calculation of Lyapunov exponents is important in finding a necessary condition for obtaining equipartition, a more direct quantity for determining if equipartition is actually reached, and also for determining the time-scale to reach it, is the information entropy (see Sects. 2.1.6 and 2.3). Along with other statistical tools, computations of the information entropy have been the backbone of the numerical observations, as we have reviewed in Sects. 2.5 and 2.6. As described in Sect. 2.3.1, the information entropy can be calculated for either modes or oscillators, with the mode description being most useful if the initial conditions are from long wavelengths (low frequencies); the numerical results are given in Sect. 2.5. The oscillator description is most useful from short-wavelength (high-frequency) initial conditions, with numerical results in Sect. 2.6.

An important early breakthrough in understanding the onset with increasing energy of observable chaos, and the approach to equipartition on numerically observable time-scales, was made by the application of Chirikov's "overlap criterion" to the FPU system by Izrailev and Chirikov. The somewhat heuristic criterion was very useful in finding transitions from localized to extensive chaos in low-dimensional chaos. Estimates in the FPU system were made both for long wavelengths and for short wavelengths to determine the "overlap" of neighboring modes. However, numerical results demonstrated that mode overlap of long-wavelength modes is not a necessary condition for equipartition but approximates a transition between weak and strong stochasticity (the SST). The result for short-wavelength modes is neither necessary nor sufficient. It predicts easy overlap at short wavelengths due to mode crowding, while numerics show consistently that equipartition is more readily obtained from long-wavelength than from short-wavelength initial conditions.

A partial resolution of the discrepancy between numerical observations of transitions to observable equipartition and the analytic "overlap" estimates came with the recognition that resonances among groups of modes would satisfy an overlap criterion at much lower energies than overlap of the neighboring modes directly. We have described the method of calculating this behavior, both for the FPU- $\beta$ chain with quartic nonlinearity, and the FPU- $\alpha$ chain with cubic nonlinearity, in Sect. 2.4.1. The results agree with numerical observations, predicting that packets of long-wavelength modes can overlap, once the energy is above a threshold, and determining scaling of the packet with 
energy and semi-quantitively determining its size. The scaling of the packet size can also be predicted by applying dimensional arguments to the partial differential equation approximation.

However, local interaction does not give a complete picture of the processes by which equipartition can be reached on numerically observable timescales. To know how energy escapes from a mode packet at low frequencies requires the understanding of the Arnold diffusion mechanism in which energy can be transferred along resonances from long-wavelength to short-wavelength modes. We have briefly described this condition in Sect. 2.4.1, but a complete understanding requires a careful reading of the original work (see [55]). Once the criterion for strong (nonexponentially slow) Arnold diffusion is well satisfied, an estimate of the scaling of the time to equipartition can be made, valid in the thermodynamic limit. We gave a summary of the estimation method in Sect. 2.8.3 which agrees well with numerical results for the FPU- $\beta$ oscillator chain, as presented in Sect. 2.5. The scaling of the equipartition time with the inverse third power of the energy density, $T_{\text {eq }} \propto \varepsilon^{-3}$ also agrees well with numerical results from the FPU- $\alpha$ chain and for the $\phi^{4}$ chain which has an onsite quartic potential.

A calculation of the Lyapunov exponent as a function of the energy density can also be made using concepts from the geometrization of Hamiltonian mechanics. We have reviewed the theory in Sect. 2.4.7 and presented the specific calculation for the FPU- $\beta$ oscillator chain in Sect. 2.8.2. A complete exposition of the geometric method and its specific application to the FPU- $\beta$ has been given in a review [42]. From the definition in (2.5), the Lyapunov exponent is determined by a long-time average, and is thus applicable from any initial condition. The comparison is most easily made with numerics starting from near-equipartition, which minimizes the effect of transients. By using this method, the scaling of $\lambda \propto \varepsilon^{2}$ was determined for the FPU- $\beta$ in the region of energy density for which $T_{\text {eq }} \propto \varepsilon^{-3}$ and the transition to the strong stochasticity regime, where the diffusion is across resonances, was also determined. Remarkably, the theoretically determined absolute values of $\lambda$ are in excellent agreement with the numerical values.

The dynamics of the evolution from short wavelengths (high frequencies) is quite different than from the long wavelengths. The evolution, starting from a high-frequency mode initial condition, occurs at higher energy and on a slower time-scale than from energy initially in a low-frequency mode. A partial understanding of the increased stability of high-frequency modes comes from the analysis of breather-like structures on discrete systems that admit exact breather solutions. High-frequency mode initial conditions have symmetry of neighboring oscillators close to that of localized exact breathers. The resulting dynamics consists of three stages. First, there is an initial stage in which the mode breaks up into a number of breather-like structures. Second, on a slower time-scale, these structures coalesce into one large unstable structure, called a chaotic breather (CB). Third, the CB slowly disintegrates, resulting in equipartition. Since a single large CB closely approximates a stable 
breather, the final decay stage, toward equipartition, can be very slow. If the energy was placed in the highest frequency mode, with a periodic boundary condition that has strict alternation of the amplitudes from one oscillator to the next, the configuration is stable up to a particular energy at which a parametric instability occurs, leading to the events described above. However, the nonlinear evolution does not depend on special initial conditions, but generically evolves from any high-frequency mode initial condition that has predominately the alternating amplitude symmetry. One does not know, in this generic situation, whether there exists any true energy threshold to achieving equipartition. However, as discussed extensively with respect to low-frequency mode initial conditions, the practical thresholds refer to observable time-scales. The scaling with energy density of the time to equipartition is estimated for high-frequency initial conditions from the beating of the breather with the background, using the procedure developed to calculate the equipartition time from low-frequency initial conditions. The result gives the numerically observed scaling to equipartition of $T_{\text {eq }} \propto \varepsilon^{-2}$, and reasonable quantitative agreement with the numerically determined values.

Considerable insight into the behavior of a nonlinear oscillator chain, starting from high-frequency mode initial conditions, can be obtained by introduction of an envelope function for the displacements of the oscillators. The initial conditions for the envelope function only contain significant long-wavelength perturbations. For the envelope function, an expansion is then possible to obtain a nonlinear partial differential equation (PDE) which approximates the behavior of the discrete system. Low-order expansions of this type produce PDEs that have integrable solutions in the form of envelope breathers, analogous to the solutions produced from low-frequency initial conditions. Higher order terms destroy the integrability, but we find, as expected, that discretized oscillator chains form localized structures that approximate the breathers on continuous systems, but are weakly unstable. The process by which the CBs coalesce into a single $\mathrm{CB}$ has also been estimated theoretically. On average, the large structures absorb energy from the smaller ones, as expected from general theoretical considerations. The time constant for coalescence into a single $\mathrm{CB}$ is estimated from the relation $\tau_{\mathrm{B}} \approx\left(n_{\mathrm{B}} \sigma v_{\mathrm{B}}\right)^{-1}$ where $n_{\mathrm{B}}$ is the breather number, $\sigma$ is a collision cross-section for absorption, and $v_{\mathrm{B}}$ is a characteristic breather velocity. Using this procedure in the numerically investigated energy range, reasonable agreement with the numerical scalings of $\tau_{\mathrm{B}} \propto \varepsilon^{-1}$ is obtained. The numerical results are presented in Sect. 2.6 and the analytic estimate for comparison to the numerics in Sect. 2.9.

To explore which processes are generic and which model dependent, it is necessary to investigate other oscillator chains which are related to the FPU chain but have significantly different parameters. The discretized KleinGordon equation with quartic nonlinearity (the $\phi^{4}$ model) is such a chain. The FPU- $\beta$ chain, with only an intersite potential, is translationally invariant (except for boundaries) and it is energy-rescalable on a single parameter. In contrast, the $\phi^{4}$ chain has an additional on-site potential which adds a 
parameter [compare (2.1) with (2.9)], and is therefore more complicated to analyze and explores a larger parameter space. Nevertheless, a comparison of the $\phi^{4}$ chain to the FPU- $\beta$ chain reveals similarities and explainable differences. For a small-value linear on-site restoring force $m=0.1$, of the individuals oscillators, the linear frequencies of most modes are similar in the $\phi^{4}$ and FPU chains. Starting from long-wavelength (low-frequency) modes the numerical calculations of $T_{\text {eq }}(\varepsilon)$ show similar behavior of the two chains, having powerlaw behavior, depending only on $\varepsilon$ over a wide range of $\varepsilon$, and $N$-dependent transitions at low $\varepsilon$ to values of $T_{\text {eq }}$ that may increase exponentially with decreasing $\varepsilon$. For larger $m$ (but $m<1$ ) the value of $\varepsilon$ required to achieve a given $T_{\text {eq }}$ is much larger with strong $m$-scaling, which can be qualitatively predicted from a heuristic argument. For short wavelengths (high frequencies) the behavior of the two chains is similar and a simple argument predicts that the same value of $T_{\text {eq }}$ can be obtained for $\phi^{4}$ as for the FPU if the $\phi^{4}$ value of $\varepsilon$ is a factor of 4 larger. The reasoning is that since the phases of neighboring oscillators alternate for the FPU, the quadratic term is factor of 16 larger, which is larger by a factor of 4 in energy; thus the energy in $\phi^{4}$ should be a factor of 4 larger to bring $T_{\text {eq }}$ into correspondence. The predicted factor of 4 separation for the higher values of $\varepsilon$ (higher $E$ ) is found when the scaling follows $\varepsilon^{-2}$ for both potentials, but the separation becomes larger as the $\phi^{4}$ potential scaling becomes steeper at low energy densities, due to additional correlations.

The knowledge that nonlinear oscillator chains, such as the FPU, produced stochastic dynamics, encouraged the idea that they would reproduce the Fourier heat law. But this was not the case. The configuration for studying heat conductivity is different from that for studying equipartition, in that the former is not a closed conservative system, but must be connected to heat baths which emit and absorb energy. Since the steady state is not an equilibrium, the dynamics involves short-time effects. The lack of normal thermal conductivity for the FPU- $\beta$ chain was found to be due to the excitation of nonlinear waves, which were not diffusive. Momentum conservation in the FPU chains is a key ingredient in preventing the stochasticity from producing the required diffusive energy flow. This led to the exploration of models which included substrate potentials, such as various Klein-Gordon models, which do produce normal heat conduction when their dynamics is primarily stochastic. The history of these developments is given in Sect. 2.1.10, and the basic theoretical ideas and numerical results are given in Sect. 2.7.

So what do we know about the generic properties of oscillator chains and their implications for physics; and what are the outstanding problems that have not been fully addressed? First, we can say that Fermi's original intuition about the role of nonlinear dynamics in underpinning statistical physics has essentially been proved correct. For large systems (approximating the thermodynamic limit) and at reasonably large energy densities, generic nonlinear oscillator chains with nearest neighbor coupling dynamically exhibit stochastic diffusion leading to equipartition among oscillators and modes in 
isolated systems. Over a wide range of energy densities the Lyapunov exponents, measuring the exponential separation of trajectories, and the resulting times to achieve equipartition are both proportional to inverse power laws of the energy density, but with different exponents. For the most thoroughly studied FPU- $\beta$ chain, these power laws have been calculated in various approximations that have yielded good agreement with numerics in the ranges of oscillator number and energy density for which computers can yield numerical results. Geometrical methods have been used to accurately predict the scaling of the Lyapunov exponent with energy density for both weak and strong diffusion, and the transition between the two regimes.

The transient dynamics, starting from a variety of initial conditions at either long or short wavelengths, can be quite complicated, but is also reasonably well understood. Again, referring to the most-studied FPU- $\beta$ chain from long-wavelength modes, the formation of mode packets on short timescales is numerically observed and theoretically understood. The relationship with soliton formation and soliton instabilities has also been established. The understanding of the role played by the web of resonance channels in phase space, and the Arnold diffusion through the web to transport energy among modes, is qualitatively understood, and the three resonance model for diffusion has been successfully used to predict the observed numerics. From shortwavelength modes, parametric instability coalescence into chaotic breathers, which ultimately dissipate, is seen numerically and the scaling of its time-scale with energy density (or energy) can be predicted. For other oscillator chains there are differences and additional complications which are generally qualitatively understood. The FPU- $\alpha$ chain is a lowest order approximation to the integral Toda chain and is therefore more stable, having a plateau in $n_{\text {eff }}$ during the time that the FPU- $\alpha$ Lyapunov exponent tracks the decay of the Lyapunov exponent for the Toda chain (whose asymptotic value is zero). The similarities and differences between the FPU- $\beta$ and $\phi^{4}$ chains are also seen numerically and qualitatively understood. These detailed dynamical processes are very interesting from the general perspective of nonlinear dynamics. However, their significance for physical problems has not been explored in any detail.

Less understood are the transitions at fixed $N$ as $\varepsilon$ is decreased. For large values of $N$, and starting from generic long-wavelength initial conditions, one expects transitions from inverse power-law scaling to exponential scaling, when the diffusion is through decreasingly thin channels. Some numerical hints of this behavior have been seen in the FPU- $\beta$ and $\phi^{4}$ chains, but the time-scales become exponentially long so that numerical results become increasingly difficult to obtain. There are also special initial conditions for which no diffusion occurs, an example being the $\pi$-mode with periodic boundary conditions for an energy below the border of parametric instability. Other situations are not so clear, particularly for small $N$, where numerical observations do not distinguish between initial conditions lying on regular orbits and lying on stochastic orbits for which the stochasticity is unobservable. 
Another area of uncertainly is the relation between the inverse power-law of $T_{\text {eq }} \propto \varepsilon^{-3}$ for various oscillator chains, and the diffusion through the stochastic channels. For the FPU- $\beta$ system, starting from long wavelengths, the universal s-shaped curve of $n_{\text {eff }}$ vs. $\log \left(\varepsilon^{3} t\right)$ (see Fig. 2.13) must consist of a complicated averaging over many increasingly fine resonance channels as equipartition is approached. No theory exists to explain the shape of this curve. The lack of explanation of the detailed evolution of this macroscopic parameter points to the difficulty in understanding detailed microscopic dynamics in problems with many degrees of freedom.

For what concerns the problem of heat conduction, the most puzzling question to be answered is the universality of the power-law divergence of heat conductivity for FPU-like chains. Since numerics is unable to yield conclusive results, one can only hope that a theoretical approach, such as rigorous hydrodynamic theory of transport in FPU-like chains, could provide the explanation.

Finally, we remark that even in the simplest many-degree-of-freedom systems, with the FPU- $\alpha$ and FPU- $\beta$ systems being prime examples, rigorous results that are also useful in making quantitative calculations are difficult to obtain. From the core material of this review, it is evident that numerical calculations underpin our understanding of the dynamics and validate our analytic calculations. For regions of the parameter space in which numerical calculations are not practical, extrapolation can be useful, but the uncertainty of relying on answers from calculations grows with increasing extrapolation and our understanding becomes less secure. Clearly, there remain challenges for theorists to obtain results that are both rigorous and useful for calculations, and for numerics to be extended into the areas of uncertainty.

\section{Acknowledgments}

We thank the University of Firenze for financially supporting the visit of AJL to Florence. SR thanks The Isaac Newton Institute in Cambridge UK for hospitality and financial support. SR thanks also Steve Greenham at Newton Institute for help in producing some of the figures. Antonio Giorgilli is acknowledged for providing us a copy of Fig. 2.14.

\section{References}

1. E. Fermi, J.R. Pasta and S. Ulam, in Collected papers of Enrico Fermi, E. Segre (ed.). University of Chicago Press, Chicago, 2, 978, 1965.

2. H. Poincaré, Les méthodes Nouvelles de la Mécanique Celeste. Gauthier-Villars, Paris, 1892.

3. E. Fermi, Zeit. Phys. 24, 261 (1923).

4. E. Fermi, Nuovo Cimento 25, 267; 26, 105 (1923). 
5. J. Ford and J. Waters, J. Math. Phys. 4, 1293 (1963).

6. E.A. Jackson, J. Math. Phys. 4, 551, 686 (1963).

7. R.L. Bivins, N. Metropolis and J.R. Pasta, J. Comp. Phys. 12, 62 (1972).

8. A.N. Kolmogorov, Dokl. Akad. Nauk. SSSR 98, 527 (1954).

9. V.I. Arnold, Soviet Math. Dokl. 2, 501 (1961).

10. J. Moser, Nachr. Akad Wiss. Gottingen, K1, 1 (1962).

11. M. Henon and C. Heiles, Astr. J. 69, 73 (1964).

12. B.V. Chirikov, Plasma Phys. (J.N.E. Pt C) 1, 253 (1960).

13. B.V. Chirikov, Phys. Rep. 52, 265 (1979).

14. V.I. Arnold, Russian Math. Surveys 18, 85 (1964).

15. A.J. Lichtenberg and M.A. Lieberman, Regular and chaotic dynamics, 2nd edition Springer, New York, 1992.

16. M. Falcioni, U.M.B. Marconi and A. Vulpiani, Phys. Rev. A 44, 2263 (1991).

17. N.N. Nekhorochev, Usp. Mat. Nauk. (USSR) 32, 6 (1977).

18. A. Giorgilli, Ann. Inst. H. Poincare Phys. Theor. 48, 423 (1988).

19. P. Lochak, Phys. Lett. A 143, 39 (1990).

20. M. Toda, Prog. Theor. Phys. Suppl. 45, 174 (1970).

21. J. Ford, S.D. Stoddard and J.S. Turner, Prog. Theor. Phys. 50, 1547 (1973).

22. M. Henon, Phys. Rev. B 9, 1925 (1974).

23. A.N. Kolmogorov, Dokl. Akad. Nauk. SSSR 124, 754 (1959).

24. Ya. G. Sinai, Dokl. Akad. Nauk. SSSR 124, 768 (1959).

25. G. Benettin, L. Galgani and J.M. Strelcyn, Phys. Rev. A 14, 2338 (1976).

26. V.I. Arnold and A. Avez, Ergodic problems of statistical mechanics. Benjamin, New York, 1968.

27. V.I. Arnold, Mathematical methods of classical mechanics. Springer-Verlag, Berlin, 1978.

28. N.S. Krylov, Works on the foundations of statistical physics, Princeton University Press, Princeton, 1979.

29. J. Hadamard, J. Math. Pur. Appl. 4, 27 (1898).

30. G.A. Hedlund, Bull. Am. Math. Soc. 45, 241 (1939).

31. E. Hopf, Proc. Nat. Acad. Sci. 18, 263 (1932).

32. V.I. Anosov, Proc. Steklov Math. Inst. 90, 1 (1967).

33. Ya.G. Sinai, Dokl. Akad. Nauk SSSR 153, 1261 (1963).

34. C.P. Ong, Adv. Math. 15, 269 (1975).

35. M.C. Gutzwiller, J. Math. Phys. 18, 806 (1977).

36. A. Knauf, Comm. Math. Phys. 110, 89 (1987).

37. M. Szydlowski, M. Heller and W. Sasin, J. Math. Phys. 37, 346 (1996).

38. H.E. Kandrup, Astrophys. J. 364, 420 (1990).

39. H.E. Kandrup, Physica A 169, 73 (1990).

40. H.E. Kandrup, Phys. Rev. E 56, 2722 (1997).

41. T.J. Hunt and MacKay, R.S., Nonlinearity 16, 1499 (2003).

42. L. Casetti, M. Pettini and E.G.D. Cohen, Phys. Rep. 337, 237 (2000).

43. M.D. Kruskal and N.J. Zabusky, J. Math. Phys. 5, 231 (1964).

44. N.J. Zabusky and M.D. Kruskal Phys. Rev. Lett. 15, 240 (1965).

45. C.F. Driscoll and T.M. O'Neil, Phys. Rev. Lett. 37, 69 (1976).

46. C.F. Driscoll and T.M. O'Neil, Rocky Mt. J. Math 5, 211 (1978).

47. F.M. Izrailev and B.V. Chirikov, Sov. Phys. Dokl. 11, 30 (1966).

48. G. Benettin, L. Galgani and A. Giorgilli, Phys. Lett. A 120, 23 (1987).

49. G. Benettin, L. Galgani and A. Giorgilli, Commun. Math. Phys. 113, 87 (1987). 
50. R. Livi, M. Pettini, S. Ruffo, M. Sparpaglione and A. Vulpiani, Phys. Rev. A 28, 3544 (1983).

51. R. Livi, M. Pettini, S. Ruffo, M. Sparpaglione and A. Vulpiani, Phys. Rev. A 31, 1039 (1985).

52. R. Livi, M. Pettini, S. Ruffo and A. Vulpiani, Phys. Rev. A 31, 2740 (1985).

53. M. Pettini and M. Landolfi, Phys. Rev. A 41, 768 (1990).

54. C.G. Goedde, A.J. Lichtenberg and M.A. Lieberman, Physica D 59, 200 (1992).

55. J. DeLuca, A.J. Lichtenberg and M.A. Lieberman, Chaos 5, 283 (1995).

56. H. Kantz, R. Livi and S. Ruffo, J. Stat. Phys. 76, 627 (1994).

57. J. DeLuca, A.J. Lichtenberg and S. Ruffo, Phys. Rev. E 51, 2877 (1995).

58. J. DeLuca, A.J. Lichtenberg and S. Ruffo, Phys. Rev. E 60, 3781 (1999).

59. L. Berchialla, A. Giorgilli and S. Paleari, Phys. Lett. A 321, 167 (2004).

60. L. Casetti, C. Clementi and M. Pettini, Phys. Rev. E 54, 5969 (1996).

61. L. Casetti, M. Cerruti-Sola, M. Pettini and E.G.D. Cohen, Phys. Rev. E 55, 6566 (1997).

62. D.L. Shepelyansky, Nonlinearity 10, 1331 (1997).

63. M. Cerruti-Sola, M. Pettini and E.G.D. Cohen, Phys. Rev. E 62, 6078 (2000).

64. M. Pettini and M. Cerruti-Sola, Phys. Rev. A 41, 44, 975 (1991).

65. J. DeLuca and A.J. Lichtenberg, Phys. Rev. E 66, 026206 (2002).

66. A. Ponno, L. Galgani and F. Guerra, Phys. Rev. E 61, 7081 (2000).

67. N.J. Zabusky and G.S. Deem, J. Comp. Phys. 2, 126 (1967).

68. N. Budinsky and T. Bountis, Physica D 8, 445 (1983).

69. S. Flach, Physica D 91, 223 (1996).

70. P. Poggi and S. Ruffo, Physica D 103, 251 (1997).

71. G.M. Chechin, N.V. Novikova and A.A. Abramenko, Physica D 166, 208 (2002).

72. G.M. Chechin, D.S. Ryabov and K.G. Zhukov, Physica D 203, 121 (2005).

73. B. Rink, Physica D 175, 31 (2003).

74. T. Dauxois, S. Ruffo and A.Torcini, Phys. Rev. E 56, R 6229 (1997).

75. V.M. Burlakov, S.A. Kiselev and V.I. Rupasov Phys. Lett. A 147, 130 (1990).

76. K.W. Sandusky and J.B. Page, Phys. Rev. B 50, 866 (1994).

77. T. Dauxois, S. Ruffo and A.Torcini, J. Phys. IV 8, 147 (1998).

78. Yu.S. Kivshar and M. Peyrard, Phys. Rev. A 46, 3198 (1992).

79. T.B. Benjamin and J.E. Feir, J. Fluid Mech. 27, 417 (1967).

80. V.E. Zakharov and A.B. Shabat Zhurnal Eksperimentalnoi I Teoreticheskoi Fiziki 64, 1627 (1973).

81. G.P. Berman and A.R. Kolovskii, Zh. Eksp. Teor. Fiz. 87, 1938 and Sov. Phys. JETP 60, 1116 (1984).

82. A.J. Sievers and S. Takeno, Phys. Rev. Lett. 61, 970 (1988).

83. V.M. Burlakov and S.A. Kiselev, Sov. Phys. JETP 72, 854 (1991).

84. S. Flach and C.R. Willis, Phys. Rep. 295, 181 (1998).

85. A.I. D'yachenko, V.E. Zackharov, A.N. Pushkarev, V.E. Shvets and V.V. Yan'kov, Sov. Phys. JETP 69, 1144 (1989).

86. O. Bang and M. Peyrard, Phys. Rev. E 53, 4143 (1996).

87. T. Cretegny, T. Dauxois, S. Ruffo and A. Torcini, Physica D 121, 109 (1998).

88. K. Ullman, A.J. Litchenberg and E. Corso, Phys. Rev E 61, 2471 (2000).

89. S. Flach, C.R. Willis and E. Olbrich, Phys. Rev. E 49, 836, (1994).

90. Yu.A. Kosevich, Phys. Rev. B 47, 3138 (1993).

91. Yu.A. Kosevich and S. Lepri, Phys. Rev. B 61, 299 (2000). 
92. V.V. Mirnov, A.J. Lichtenberg and H. Guclu, Physica D 157, 251 (2001).

93. A.J. Lichtenberg and V.V. Mirnov, Physica D, 202, 116 (2005).

94. A.J. Lichtenberg, V.V. Mirnov and C. Day, Chaos, 15, 015109 (2005).

95. M.C. Forrest, C.G. Goedde and S. Sinha, Phys. Rev. Lett. 68, 2722 (1992).

96. J.L. Marin and S. Aubry, Physica D, 119, 163 (1998).

97. S.R. de Groot and P. Mazur, Non-equilibrium thermodynamics, Dover, NY, 1984.

98. P. Debye, Communication at the congress on the kinetic theory of matter. Gottingen, 1913.

99. R.E. Peierls, Quantum theory of solids. Oxford University Press (1955).

100. Z. Rieder, J.L. Lebowitz and E. Lieb, J. Math. Phys. 8, 1073 (1967).

101. S. Lepri, R. Livi and A. Politi, Phys. Rep. 377, 1 (2003).

102. D.N. Payton, M. Rich and W.M. Visscher, Phys. Rev. 160, 706 (1967).

103. E.A. Jackson, J.R. Pasta and J.F. Waters, J. Comput. Phys. 2, 207 (1968).

104. N. Nakazawa, Progr. Theor. Phys. (Suppl. 45), 231 (1970).

105. W.M. Visscher, Methods in computational physics Vol. 15, p. 371. Academic Press, New York, 1976.

106. H. Kaburaki and M. Machida, Phys. Lett. A 181, 85 (1993).

107. Y. Ohtsuboi, N. Nishiguchi and T. Sakuma, J. Phys. Condens. Matter 6, 3013 (1994).

108. F. Mokross and H. Buttner, J. Phys. C 16, 4539 (1983).

109. E.A. Jackson and A.D. Mistriotis, J. Phys. Cond. Mat. 1, 1223 (1984).

110. N. Nishiguchi and T. Sakuma, J. Phys. Condens. Matter 2, 7575 (1990).

111. O.V. Gendelman and L.I. Manevich, Sov. Phys. JETP 75, 271 (1992).

112. S. Lepri, R. Livi and A. Politi, Phys. Rev. Lett. 78, 1896 (1997).

113. S. Lepri, R. Livi and A. Politi, Physica D 119, 140 (1998).

114. S. Lepri, R. Livi and A. Politi, Europhys. Lett. 43, 271 (1998).

115. S. Lepri, Eur. Phys. J. B 18, 441 (2000).

116. T. Hatano, Phys. Rev. E 59, R1 (1999).

117. M. Vassalli, Diploma Thesis, University of Florence, 1999.

118. L. Galgani, A. Giorgilli, A. Martinoli and S. Vanzini, Physics D 59, 334 (1992).

119. G. Casati, J. Ford , F. Vivaldi and W.M. Visscher, Phys. Rev. Lett. 52, 1861 (1984).

120. T. Prozen and D.K. Campbell, Chaos 15, D15117 (2005).

121. J. De Luca and A.J. Lichtenberg, Phys. Rev. E. 66, 026206 (2002).

122. D. Sholl, Phys. Lett. A 149, 253 (1990).

123. R. Livi and A. Vulpiani, (eds), L' Heritage de Kolmogorov en Physique, Editions Belin, Paris; The Kolmogorv Legacy in Physics Lectures Notes in Physics, Springer, Berlin and New York, 2003.

124. J.P. Eckmann and D. Ruelle, Rev. Mod. Phys. 57, 617 (1985).

125. M. Tabor, Chaos and integrability in nonlinear dynamics, John Wiley \& Sons. Inc., New York, 1989.

126. E. Ott, Chaos in dynamical systems. Cambridge University Press, Cambridge 1993.

127. Ya. B. Pesin, Russ. Math. Surveys 32, 55 (1977).

128. R. Livi, A. Politi and S. Ruffo, J. Phys. A 19, 2033 (1986).

129. J.P. Eckmann and E. Wayne, J. Stat. Phys. 50, 853 (1988).

130. Ya.G. Sinai, Int. J. Bifur. Chaos 6, 1137 (1996).

131. L. Casetti, R. Livi and M. Pettini, Phys. Rev. Lett. 74, 375 (1995). 
132. S. Isola, R. Livi, S. Ruffo and A. Vulpiani, Phys. Rev. A 33, 1163 (1986).

133. S. Flach, M.V. Ivanchenko and O.I. Kanakov, Phys. Rev. Lett. 95, 064102 (2005).

134. J. DeLuca, A.J. Lichtenberg and S. Ruffo, Phys. Rev. E 54, 2329 (1996).

135. A. Ponno and Bambusi D., Energy cascade in Fermi-Pasta-Ulam models, Proceedings of the International Conference on Symmetry and Perturbation Theory 2004, G. Gaeta et al. (eds), World Scientific Publishing, 263-270 and private communication, 2005.

136. J.A. Biello, P.R. Kramer, Y.V. L'vov, Discr. Cont. Dynam. Sys. (Suppl. 113) (2003).

137. L. Berchialla, Galgani L. and Giorgilli A., Discr. Cont. Dynam. Sys. (Suppl., 855), (2005).

138. T. Dauxois, R. Khomeriki, F. Piazza and S. Ruffo, Chaos 15, 015110 (2005).

139. A. Cafarella, M. Leo and R.A. Leo, Phys. Rev. E 69, 046604 (2004).

140. G.P. Berman, F.M. Izrailev, Chaos, 15, 015104 (2005).

141. L. Caiani, L. Casetti and M. Pettini, J. Phys. A 31, 3357 (1998).

142. M. Pettini, Phys. Rev. E 47, 828 (1993).

143. M. Cerruti-Sola and M. Pettini, Phys. Rev. E 51, 53 (1995).

144. M. Cerruti-Sola and M. Pettini, Phys. Rev. E 53, 179 (1996).

145. A. Abraham and J.E. Marsden, Foundations of mechanics. Addison-Wesley, Redwood City, 1987.

146. M.P. do Carmo, Riemannian geometry. Birkhäuser, Boston-Basel, 1993.

147. L.P. Eisenhart, Ann. of Math. 30, 591 (1929).

148. J. Guckenheimer and P. Holmes, Nonlinear oscillations, dynamical systems and bifurcations of vector fields, ed. Springer, New York, 1983.

149. M. Pettini and R. Valdettaro, Chaos 5, 646 (1995).

150. N.G. Van Kampen, Phys. Rep. 24, 71 (1976).

151. R. Livi, M. Pettini, S. Ruffo and A. Vulpiani, J. Stat. Phys. 48, 539 (1987).

152. G. Benettin, L. Galgani, A. Giorgilli and J.M. Strelcyn, Meccanica 15, 9 and 21 (1980).

153. G. Benettin, L. Galgani and A. Giorgilli, Il Nuovo Cimento B 89, 89 (1985).

154. R. Kubo, M. Toda and N. Hashitsume, Statistical physics II. Springer Series in Solid State Sciences, Vol. 31, Springer, Berlin, 1991.

155. K. Aoki and D. Kusnezov, Phys. Rev. Lett. 86, 4029 (2001).

156. D. Escande, H. Kantz, R. Livi and S. Ruffo, J. Stat. Phys. 76, 605 (1994).

157. C. Giardinà, R. Livi, A. Politi and M. Vassalli, Phys. Rev. Lett. 84, 2144 (2000).

158. O.V. Gendelman and A.V. Savin, Phys. Rev. Lett. 84, 2381 (2000).

159. J. Dawson, Phys. Fluids 5, 4451962.

160. G. Casati, Found. Phys. 16, 51 (1986).

161. T. Prozen and M. Robnik, J. Phys. A 25, 3449 (1992).

162. D.J.R. Mimnagh and L.E. Ballentine, Phys. Rev. E 56, 5332 (1997).

163. H.A. Posch and W.G. Hoover, Phys. Rev. E 58, 4344 (1998).

164. M.J. Gillan and R.W. Holloway, J. Phys. C 18, 5705 (1985).

165. B. Hu, B. Li and H. Zhao, Phys. Rev. E 57, 2992 (1998).

166. A. Filippov, B. Hu, B. Li and A. Zelser, J. Phys. A 31, 7719 (1998).

167. B. Hu, B. Li and H. Zhao, Phys. Rev. E 61, 3828 (1999).

168. K. Aoki and D. Kusnezov, Phys. Lett. A 265, 250 (2000).

169. G.P. Tsironis, A.R. Bishop, A.V. Savin and A.V. Zolotaryuk, Phys. Rev. E 60, 6610 (1999). 
170. G. Tsaur and J. Wang, Phys. Rev. E 54, 4657 (1996).

171. V.I. Oseledec, Trans. Moscow Math. Soc. 19, 197 (1968).

172. J.L. Lebowitz, Percus J.K. and Verlet L., Phys. Rev. 153, 250 (1967).

173. L. Caiani, L. Casetti, C. Clementi and M. Pettini, Phys. Rev. Lett. 79, 4361 (1997).

174. L. Caiani, L. Casetti, C. Clementi, G. Pettini, M. Pettini and R. Gatto, Phys. Rev. E 57, 3886 (1998).

175. M.-C. Firpo, Phys. Rev. E 57, 6599 (1998). 
